\title{
Cover times, blanket times, and majorizing measures
}

\author{
Jian Ding* \\ U. C. Berkeley
}

\author{
James R. Lee ${ }^{\dagger *}$ \\ University of Washington
}

\author{
Yuval Peres \\ Microsoft Research
}

\begin{abstract}
We exhibit a strong connection between cover times of graphs, Gaussian processes, and Talagrand's theory of majorizing measures. In particular, we show that the cover time of any graph $G$ is equivalent, up to universal constants, to the square of the expected maximum of the Gaussian free field on $G$, scaled by the number of edges in $G$.

This allows us to resolve a number of open questions. We give a deterministic polynomialtime algorithm that computes the cover time to within an $O(1)$ factor for any graph, answering a question of Aldous and Fill (1994). We also positively resolve the blanket time conjectures of Winkler and Zuckerman (1996), showing that for any graph, the blanket and cover times are within an $O(1)$ factor. The best previous approximation factor for both these problems was $O\left((\log \log n)^{2}\right)$ for $n$-vertex graphs, due to Kahn, Kim, Lovász, and Vu (2000).
\end{abstract}

\section{Contents}

1 Introduction $\quad 2$

1.1 Related work . . . . . . . . . . . . . . . . . . . 6

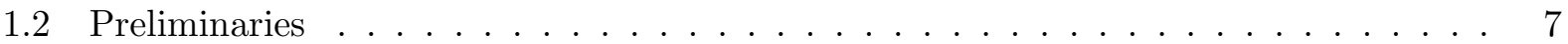

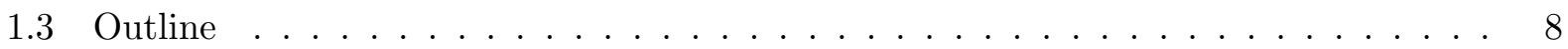

2 Gaussian processes and local times

2.1 The blanket time . . . . . . . . . . . . . . . . . . . 12

2.2 An asymptotically strong upper bound . . . . . . . . . . . . . 17

2.3 Geometry of the resistance metric . . . . . . . . . . . . . . 18

2.4 The Gaussian free field . . . . . . . . . . . . . . . . . . 22

3 Majorizing measures

3.1 Trees, measures, and functionals . . . . . . . . . . . . . . 25

3.2 Separated trees . . . . . . . . . . . . . . . . . . 27

3.3 Computing an approximation to $\gamma_{2}$ deterministically $\ldots \ldots \ldots \ldots \ldots \ldots$

3.4 Tree-like properties of the Gaussian free field . . . . . . . . . . . . . . . 34

* A substantial portion of this work was completed during visits of the author to Microsoft Research.

${ }^{\dagger}$ Partially supported by NSF grant CCF-0915251 and a Sloan Research Fellowship. 
4 The cover time 36

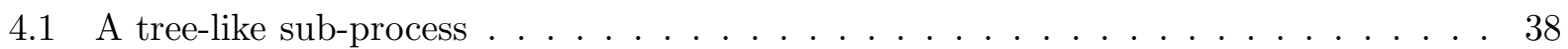

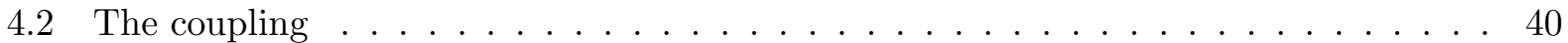

4.3 Tree-like percolation . . . . . . . . . . . . . . . . . . . 47

4.4 The local times . . . . . . . . . . . . . . . . . . . . . . . . . . . . 48

4.5 Additional applications . . . . . . . . . . . . . . . . . . . . 49

5 Open problems and further discussion $\quad 51$

\section{Introduction}

Let $G=(V, E)$ be a finite, connected graph, and consider the simple random walk on $G$. Writing $\tau_{\text {cov }}$ for the first time at which every vertex of $G$ has been visited, let $\mathbb{E}_{v} \tau_{\text {cov }}$ denote the expectation of this quantity when the random walk is started at some vertex $v \in V$. The following fundamental parameter is known as the cover time of $G$,

$$
t_{\mathrm{cov}}(G)=\max _{v \in V} \mathbb{E}_{v} \tau_{\mathrm{cov}}
$$

We refer to the books [2, 36] and the survey [37] for relevant background material.

We also recall the discrete Gaussian free field $(\mathrm{GFF})$ on the graph $G$. This is a centered Gaussian process $\left\{\eta_{v}\right\}_{v \in V}$ with $\eta_{v_{0}}=0$ for some fixed $v_{0} \in V$. The process is characterized by the relation $\mathbb{E}\left(\eta_{u}-\eta_{v}\right)^{2}=R_{\text {eff }}(u, v)$ for all $u, v \in V$, where $R_{\text {eff }}$ denotes the effective resistance on $G$. Equivalently, the covariances $\mathbb{E}\left(\eta_{u} \eta_{v}\right)$ are given by the Green kernel of the random walk killed at $v_{0}$. (We refer to Sections 1.2 and 1.3 for background on electrical networks and Gaussian processes.)

The next theorem represents one of the primary connections put forward in this work. We use the notation $\asymp$ to denote equivalence up to a universal constant factor.

Theorem 1.1. For any finite, connected graph $G=(V, E)$, we have

$$
t_{\mathrm{cov}}(G) \asymp|E|\left(\mathbb{E} \max _{v \in V} \eta_{v}\right)^{2},
$$

where $\left\{\eta_{v}\right\}_{v \in V}$ is the Gaussian free field on $G$.

The utility of such a characterization will become clear soon. Despite being an intensively studied parameter of graphs, a number of basic questions involving the cover time have remained open. We now highlight two of these, whose resolution we discuss subsequently.

The blanket time. For a node $v \in V$, let $\pi(v)=\frac{\operatorname{deg}(v)}{2|E|}$ denote the stationary measure of the random walk, and let $N_{v}(t)$ be a random variable denoting the number of times the random walk has visited $v$ up to time $t$. Now define $\tau_{\text {bl }}^{\circ}(\delta)$ to be the first time $t \geqslant 1$ at which

$$
N_{v}(t) \geqslant \delta t \pi(v)
$$

holds for all $v \in V$. In other words, $\tau_{\mathrm{bl}}^{\circ}(\delta)$ is the first time at which all nodes have been visited at least a $\delta$ fraction as much as we expect at stationarity. Using the same notation as in (11), define the $\delta$-blanket time as

$$
t_{\mathrm{bl}}^{\circ}(G, \delta)=\max _{v \in V} \mathbb{E}_{v} \tau_{\mathrm{bl}}^{\circ}(\delta) .
$$


Clearly for $\delta \in(0,1)$, we have $t_{\mathrm{bl}}^{\circ}(G, \delta) \geqslant t_{\mathrm{cov}}(G)$. Winkler and Zuckerman [54] made the following conjecture.

Conjecture 1.1. For every $0<\delta<1$, there exists a $C$ such that for every graph $G$, one has

$$
t_{\mathrm{bl}}^{\circ}(G, \delta) \leqslant C \cdot t_{\mathrm{cov}}(G)
$$

In other words, for every fixed $\delta \in(0,1)$, one has $t_{\mathrm{cov}}(G) \asymp t_{\mathrm{bl}}^{\circ}(G, \delta)$.

Kahn, Kim, Lovász, and $\mathrm{Vu}$ [30] showed that for every fixed $\delta \in(0,1)$, one can take $C \asymp$ $(\log \log n)^{2}$ for $n$-node graphs, but whether there is a universal constant, independent of $n$, remained open for every value of $\delta>0$.

In order to bound $t_{\mathrm{bl}}^{\circ}(G, \delta)$, we introduce the following stronger notion. Let $\tau_{\mathrm{bl}}(\delta)$ be the first time $t \geqslant 1$ such that for every $u, v \in V$, we have

$$
\frac{N_{u}(t) / \pi(u)}{N_{v}(t) / \pi(v)} \geqslant \delta
$$

i.e. the first time at which all the values $\left\{N_{u}(t) / \pi(u)\right\}_{u \in V}$ are within a factor of $\delta$. As in [30], we define the strong $\delta$-blanket time as

$$
t_{\mathrm{bl}}(G, \delta)=\max _{v \in V} \mathbb{E}_{v} \tau_{\mathrm{bl}}(\delta) .
$$

Clearly one has $t_{\mathrm{bl}}^{\circ}(G, \delta) \leqslant t_{\mathrm{bl}}(G, \delta)$ for every $\delta \in(0,1)$.

The second question we highlight is computational in nature.

Question 1.2 ([2, 30]). Is there a deterministic, polynomial-time algorithm that approximates $t_{\mathrm{cov}}(G)$ within a constant factor?

In other words, is there a quantity $A(G)$ which can be computed deterministically, in polynomialtime in $|V|$, such that $A(G) \asymp t_{\text {cov }}(G)$. It is crucial that one asks for a deterministic procedure, since a randomized algorithm can simply simulate the chain, and output the empirical mean of the observed times at which the graph is first covered. This is guaranteed to produce an accurate estimate with high-probability in polynomial time, since the mean and standard deviation of $\tau_{\text {cov }}$ are $O\left(|V|^{3}\right)[6$.

A result of Matthews [43] can be used to produce a determinisically computable bound which is within a $\log |V|$ factor of $t_{\text {cov }}(G)$. Subsequently, 30] showed how one could compute a bound which lies within an $O\left((\log \log |V|)^{2}\right)$ factor of the cover time.

Before we state our main theorem and resolve the preceding questions, we briefly review the $\gamma_{2}$ functional from Talagrand's theory of majorizing measures [48, 50].

Majorizing measures and Gaussian processes. Consider a compact metric space $(X, d)$. Let $M_{0}=1$ and $M_{k}=2^{2^{k}}$ for $k \geqslant 1$. For a partition $P$ of $X$ and an element $x \in X$, we will write $P(x)$ for the unique $S \in P$ containing $x$. An admissible sequence $\left\{A_{k}\right\}_{k \geqslant 0}$ of partitions of $X$ is such that $A_{k+1}$ is a refinement of $A_{k}$ for $k \geqslant 0$, and $\left|A_{k}\right| \leqslant M_{k}$ for all $n \geqslant 0$. Talagrand defines the functional

$$
\gamma_{2}(X, d)=\inf \sup _{x \in X} \sum_{k \geqslant 0} 2^{k / 2} \operatorname{diam}\left(A_{k}(x)\right)
$$


where the infimum is over all admissible sequences $\left\{A_{k}\right\}$.

Consider now a Gaussian process $\left\{\eta_{i}\right\}_{i \in I}$ over some index set $I$. This is a stochastic process such that every finite linear combination of random variables is normally distributed. For the purposes of the present paper, one may assume that $I$ is finite. We will assume that all Gaussian processes are centered, i.e. $\mathbb{E}\left(\eta_{i}\right)=0$ for all $i \in I$. The index set $I$ carries a natural metric which assigns, for $i, j \in I$,

$$
d(i, j)=\sqrt{\mathbb{E}\left|\eta_{i}-\eta_{j}\right|^{2}} .
$$

The following result constitutes a primary consequence of the majorizing measures theory.

Theorem (MM) (Majorizing measures theorem [48]). For any centered Gaussian process $\left\{\eta_{i}\right\}_{i \in I}$,

$$
\gamma_{2}(I, d) \asymp \mathbb{E} \sup \left\{\eta_{i}: i \in I\right\} .
$$

We remark that the upper bound of the preceding theorem, i.e. $\mathbb{E} \sup \left\{\eta_{i}: i \in I\right\} \leqslant C \gamma_{2}(I, d)$ for some constant $C$, goes back to work of Fernique [24, 25]. Fernique formulated this result in the language of measures (from whence the name "majorizing measures" arises), while the formulation of $\gamma_{2}$ given in (4) is due to Talagrand. The fact that the two notions are related is non-trivial; we refer to [50, §2] for a thorough discussion of the connection between them.

Commute times, hitting times, and cover times. In order to relate the majorizing measure theory to cover times of graphs, we recall the following natural metric. For any two nodes $u, v \in V$, use $H(u, v)$ to denote the expected hitting time from $u$ to $v$, i.e. the expected time for a random walk started at $u$ to hit $v$. The expected commute time between two nodes $u, v \in V$ is then defined by

$$
\kappa(u, v)=H(u, v)+H(v, u) .
$$

It is immediate that $\kappa(u, v)$ is a metric on any finite, connected graph. A well-known fact [11] is that $\kappa(u, v)=2|E| R_{\mathrm{eff}}(u, v)$, where $R_{\mathrm{eff}}(u, v)$ is the effective resistance between $u$ and $v$, when $G$ is considered as an electrical network with unit conductances on the edges. We now restate our main result in terms of majorizing measures. For a metric $d$, we write $\sqrt{d}$ for the distance $\sqrt{d}(u, v)=\sqrt{d(u, v)}$.

Theorem 1.2 (Cover times, blanket times, and majorizing measures). For any graph $G=(V, E)$ and any $0<\delta<1$, we have

$$
t_{\mathrm{cov}}(G) \asymp\left[\gamma_{2}(V, \sqrt{\kappa})\right]^{2}=|E| \cdot\left[\gamma_{2}\left(V, \sqrt{R_{\mathrm{eff}}}\right)\right]^{2} \asymp_{\delta} t_{\mathrm{bl}}(G, \delta),
$$

where $\asymp_{\delta}$ denotes equivalence up to a constant depending on $\delta$.

Clearly this yields a positive resolution to Conjecture 1.1. Moreover, we prove the preceding theorem in the setting of general finite-state reversible Markov chains. See Theorem 1.9 for a statement of our most general theorem.

We now address some additional consequences of the main theorem. First, observe that by combining Theorem 1.2 with Theorem (MM), we obtain Theorem 1.1

Theorem 1.3 (Cover times and the Gaussian free field). For any graph $G=(V, E)$ and any $0<\delta<1$, we have

$$
t_{\mathrm{cov}}(G) \asymp|E|\left(\mathbb{E} \max _{v \in V} \eta_{v}\right)^{2} \asymp \delta t_{\mathrm{bl}}(G, \delta),
$$

where $\left\{\eta_{v}\right\}$ is the Gaussian free field on $G$. 
In fact, in Section 2.2, we exhibit the following strong asymptotic upper bound.

Theorem 1.4. For every graph $G=(V, E)$, if $t_{\text {hit }}(G)$ denotes the maximal hitting time in $G$, and $\left\{\eta_{v}\right\}_{v \in V}$ is the Gaussian free field on $G$, then

$$
t_{\mathrm{cov}}(G) \leqslant\left(1+C \sqrt{\frac{t_{\mathrm{hit}}(G)}{t_{\mathrm{cov}}(G)}}\right) \cdot|E| \cdot\left(\mathbb{E} \sup _{v \in V} \eta_{v}\right)^{2},
$$

where $C>0$ is a universal constant.

In Section 3, we prove the following theorem which, in conjunction with Theorem 1.2, resolves Question 1.2.

Theorem 1.5. Let $(X, d)$ be a finite metric space, with $n=|X|$. If, for any two points $x, y \in X$, one can deterministically compute $d(x, y)$ in time polynomial in $n$, then one can deterministically compute a number $A(X, d)$ in polynomial time, for which

$$
A(X, d) \asymp \gamma_{2}(X, d) .
$$

A "comparison theorem" follows immediately from Theorem 1.2 , and the fact that $\gamma_{2}(X, d) \leqslant$ $L \gamma_{2}\left(X, d^{\prime}\right)$ whenever $d \leqslant L d^{\prime}$ (see (44)).

Theorem 1.6 (Comparison theorem for cover times). Suppose $G$ and $G^{\prime}$ are two graphs on the same set of nodes $V$, and $\kappa_{G}$ and $\kappa_{G^{\prime}}$ are the distances induced by respective commute times. If there exists a number $L \geqslant 1$ such that $\kappa_{G}(u, v) \leqslant L \cdot \kappa_{G^{\prime}}(u, v)$ for all $u, v \in V$, then

$$
t_{\mathrm{cov}}(G) \leqslant O(L) \cdot t_{\mathrm{cov}}\left(G^{\prime}\right) .
$$

Finally, our work implies that there is an extremely simple randomized algorithm for computing the cover time of a graph, up to constant factors. To this end, consider a graph $G=(V, E)$ whose vertex set we take to be $V=\{1,2, \ldots, n\}$. Let $D$ be the diagonal degree matrix, i.e. such that $D_{i i}=\operatorname{deg}(i)$ and $D_{i j}=0$ for $i \neq j$, and let $A$ be the adjacency matrix of $G$. We define the following normalized Laplacian,

$$
L_{G}=\frac{D-A}{\operatorname{tr}(D)} .
$$

Let $L_{G}^{+}$denote the Moore-Penrose peudoinverse of $L_{G}$. Note that both $L_{G}$ and $L_{G}^{+}$are positive semi-definite. We have the following characterization.

Theorem 1.7. For any connected graph $G$, it holds that

$$
t_{\mathrm{cov}}(G) \asymp \mathbb{E}\left\|\sqrt{L_{G}^{+}} g\right\|_{\infty}^{2}
$$

where $g=\left(g_{1}, \ldots, g_{n}\right)$ is an $n$-dimensional Gaussian, i.e. such that $\left\{g_{i}\right\}$ are i.i.d. $N(0,1)$ random variables.

The preceding theorem yields an $O\left(n^{\omega}\right)$-time randomized algorithm for approximating $t_{\text {cov }}(G)$, where $\omega \in[2,2.376)$ is the best-possible exponent for matrix multiplication [13. Using the linearsystem solvers of Spielman and Teng [47] (see also [45]), along with ideas from Spielman and Srivistava [46, we present an algorithm that runs in near-linear time in the number of edges of $G$.

Theorem 1.8 (Near-linear time randomized algorithm). There is a randomized algorithm which, given an $m$-edge connected graph $G=(V, E)$, runs in time $O\left(m(\log m)^{O(1)}\right)$ and outputs a number $A(G)$ such that $t_{\mathrm{cov}}(G) \asymp \mathbb{E}[A(G)] \asymp\left(\mathbb{E}\left[A(G)^{2}\right]\right)^{1 / 2}$. 


\section{$1.1 \quad$ Related work}

Cover times of finite graphs have been studied for over 30 years. We refer to [2, 37, 36] for the basic theory. Works of Feige showed that the cover time for any $n$-node graph is at least $(1-o(1)) n \log n$ [22], and at most $4 n^{3} / 27$ [21]. Both of these bounds are asymptotically tight, with the tight example for the lower bound given by the complete graph on $n$ nodes.

The connection between cover times, commute times, and the theory of electrical networks was laid out in [11]. In general, the electrical viewpoint provides a powerful methodology for analyzing random walks (see, for example, [15, 53, 39]). Indeed, this point of view will be central to the present work.

A fundamental bound of Matthews [43] shows that

$$
t_{\mathrm{cov}}(G) \leqslant\left(\max _{u, v \in V} H(u, v)\right)(1+\log n)
$$

where we recall that $H(u, v)$ is the expected hitting time from $u$ to $v$. Using the straightforward lower bound $t_{\mathrm{cov}}(G) \geqslant \max _{u, v \in V} H(u, v)$, this fact provides a deterministic $O(\log n)$-approximation to $t_{\text {cov }}(G)$ in $n$-node graphs.

Matthews also proved the lower bound,

$$
t_{\mathrm{cov}}(G) \geqslant \max _{S \subseteq V}\left(\min _{u \neq v \in S} H(u, v)\right) \log (|S|-1) .
$$

In [30], it is shown that taking the maximum of the lower bound in (7) and the maximal hitting time $\max _{u, v \in V} H(u, v)$ is an $O\left((\log \log n)^{2}\right)$-approximation for $t_{\text {cov }}$. Recently, Feige and Zeitouni 23. have shown that on trees, one can obtain a very strong bound: For every $\varepsilon>0$, there is a $(1+\varepsilon)$-approximation obtainable by a deterministic, polynomial-time algorithm.

The cover time has also been studied for many specific families of graphs. Kahn, Linial, Nisan, and Saks [31] established an $O\left(n^{2}\right)$ upper bound for regular graphs. Broder and Karlin [9] proved that the cover time of constant-degree expander graphs is $O(n \log n)$. For planar graphs of maximum degree $d$, Jonasson and Schramm [29] showed that the cover time is at least $c_{d} n(\log n)^{2}$ and at most $6 n^{2}$. The order of the cover time on lattices was determined by Aldous [1] and Zuckerman [55]. The latter paper also calculated the order of the cover time on regular trees.

Furthermore, for a few families of specific examples, the asymptotics of the cover time have been calculated more precisely. These include the work of Aldous [4 for regular trees, Dembo, Peres, Rosen, and Zeitouni [14] for the 2-dimensional discrete torus, and Cooper and Frieze [12] for the giant component of various random graphs.

Finally, we remark on an upper bound of Barlow, Ding, Nachmias, and Peres [7] which was part of the motivation for the present work. Consider a connected graph $G=(V, E)$ and the metric space $(V, \kappa)$, where we recall the commute distance from ([6). For each $h \in \mathbb{Z}$, let $A_{h} \subseteq V$ be a set of minimal size whose $2^{h}$-neighborhood (in the metric $\kappa$ ) covers $V$. Then,

$$
t_{\mathrm{cov}}(G) \leqslant O(1) \cdot\left(\sum_{h \in \mathbb{Z}} 2^{h / 2} \sqrt{\log \left|A_{h}\right|}\right)^{2} .
$$

It turns out that this upper bound is tight (up to a universal constant) for a number of concrete examples with approximately "homogeneous" geometry (we refer to [7] for examples, mostly related 
to various random graphs arising from percolation). For instance, the results of the present paper imply that the right-hand side of (8) is equivalent to $t_{\text {cov }}(G)$ for any vertex-transitive graph $G$. Furthermore, the formula (8) resembles the appearance of the Dudley integral [16], which gives a tight bound for Gaussian processes with stationary increments. This suggests, in particular, a connection between the cover time of graphs and majorizing measures.

\subsection{Preliminaries}

To begin, we introduce some fundamental notions from random walks and electrical networks.

Electrical networks and random walks. A network is a finite, undirected graph $G=(V, E)$, together with a set of non-negative conductances $\left\{c_{x y}: x, y \in V\right\}$ supported exactly on the edges of $G$, i.e. $c_{x y}>0 \Longleftrightarrow x y \in E$. The conductances are symmetric so that $c_{x y}=c_{y x}$ for all $x, y \in V$. We will write $c_{x}=\sum_{y \in V} c_{x y}$ and $\mathcal{C}=\sum_{x \in V} c_{x}$ for the total conductance. We will often use the notation $G(V)$ for a network on the vertex set $V$. In this case, the associated conductances are implicit. In the few cases when there are multiple networks under consideration simultaneously, we will use the notation $c_{x y}^{G}$ to refer to the conductances in $G$.

Associated to such a network is the canonical discrete time random walk on $G$, whose transition probabilities are given by $p_{x y}=c_{x y} / c_{x}$ for all $x, y \in V$. It is easy to see that this defines the transition matrix of a reversible Markov chain on $V$, and that every finite-state reversible Markov chain arises in this way (see [2, §3.2]). The stationary measure of a vertex is precisely $\pi(x)=c_{x} / \mathcal{C}$.

Associated to such an electrical network are the classical quantities $C_{\text {eff }}, R_{\text {eff }}: V \times V \rightarrow \mathbb{R}_{\geqslant 0}$ which are referred to, respectively, as the effective conductance and effective resistance between pairs of nodes. We refer to [36, Ch. 9] for a discussion of the connection between electrical networks and the corresponding random walk. For now, it is useful to keep in mind the following fact [11]: For any $x, y \in V$,

$$
R_{\mathrm{eff}}(x, y)=\frac{\kappa(x, y)}{\mathcal{C}}
$$

where the commute time $\kappa$ is defined as before (6) $)$.

For convenience, we will work exclusively with continuous-time Markov chains, where the transition rates between nodes are given by the probabilities $p_{x y}$ from the discrete chain. One way to realize the continuous-time chain is by making jumps according to the discrete-time chain, where the times spent between jumps are i.i.d. exponential random variables with mean 1 . We refer to these random variables as the holding times. See [2, Ch. 2] for background and relevant definitions.

Cover times, local times, and blanket times. We will now define various stopping times for the continuous-time random walk. First, we observe that if $\tau_{\text {cov }}^{\star}$ is the first time at which the continuous-time random walk has visited every node of $G$, then for every vertex $v$,

$$
\mathbb{E}_{v} \tau_{\mathrm{cov}}^{\star}=\mathbb{E}_{v} \tau_{\mathrm{cov}}
$$

where we recall that the latter quantity refers to the discrete-time chain. Thus we may also define the cover time with respect to the continuous-time chain, i.e. $t_{\text {cov }}(G)=\max _{v \in V} \mathbb{E}_{v} \tau_{\text {cov }}^{\star}$.

In fact, it will be far more convenient to work with the cover and return time defined as follows. Let $\left\{X_{t}\right\}_{t \in[0, \infty)}$ be the continuous-time chain, and define

$$
\tau_{\mathrm{cov}}^{\circlearrowright}=\inf \left\{t>\tau_{\mathrm{cov}}^{\star}: X_{t}=X_{0}\right\} .
$$


For concreteness, we define the cover and return time of $G$ as

$$
t_{\mathrm{cov}}^{\circlearrowright}(G)=\max _{v \in V} \mathbb{E}_{v} \tau_{\mathrm{cov}}^{\circlearrowright}
$$

but the following fact shows that the choice of initial vertex is not of great importance for us (see [2, Ch. 5, Lem. 25]),

$$
\frac{1}{2} t_{\mathrm{cov}}^{\circlearrowright}(G) \leqslant t_{\mathrm{cov}}(G) \leqslant t_{\mathrm{cov}}^{\circlearrowright}(G) \leqslant 3 \min _{v \in V} \mathbb{E}_{v} \tau_{\mathrm{cov}}^{\circlearrowright}
$$

For a vertex $v \in V$ and time $t$, we define the local time $L_{t}^{v}$ by

$$
L_{t}^{v}=\frac{1}{c_{v}} \int_{0}^{t} \mathbf{1}_{\left\{X_{s}=v\right\}} d s
$$

where we recall that $c_{v}=\sum_{u \in V} c_{u v}$. For $\delta \in(0,1)$, we define $\tau_{\mathrm{bl}}^{\star}(\delta)$ as the first time $t>0$ at which

$$
\min _{u, v \in V} \frac{L_{t}^{u}}{L_{t}^{v}} \geqslant \delta
$$

Furthermore, the continuous-time strong $\delta$-blanket time is defined to be

$$
t_{\mathrm{bl}}^{\star}(G, \delta)=\max _{v \in V} \mathbb{E}_{v} \tau_{\mathrm{bl}}^{\star}(\delta) .
$$

Asymptotic notation. For expressions $A$ and $B$, we will use the notation $A \lesssim B$ to denote that $A \leqslant C \cdot B$ for some constant $C>0$. If we wish to stress that the constant $C$ depends on some parameter, e.g. $C=C(p)$, we will use the notation $A \lesssim p B$. We use $A \asymp B$ to denote the conjunction $A \lesssim B$ and $B \lesssim A$, and we use the notation $A \asymp_{p} B$ similarly.

\subsection{Outline}

We first state our main theorem in full generality. We use only the language of effective resistances, since this is most natural in the context to follow.

Theorem 1.9. For any network $G=(V, E)$ and any $0<\delta<1$,

$$
t_{\mathrm{cov}}(G) \asymp \mathcal{C}\left[\gamma_{2}\left(V, \sqrt{R_{\mathrm{eff}}}\right)\right]^{2} \asymp_{\delta} t_{\mathrm{bl}}(G, \delta) \asymp_{\delta} t_{\mathrm{bl}}^{\star}(G, \delta),
$$

where $\mathcal{C}$ is the total conductance of $G$.

We now present an overview of our main arguments, and layout the organization of the paper.

Hints of a connection. First, it may help the reader to have some intuition about why cover times should be connected to the Gaussian processes and particularly the theory of majorizing measures.

A first hint goes back to work of Aldous [3], where it is shown that the hitting times of Markov chains are approximately distributed as exponential random variables. It is well-known that an exponential variable can be represented as the sum of the squares of two Gaussians. Observing that the cover time is just the maximum of all the hitting times, one might hope that the cover time can be related to the maximum of a family of Gaussians. 
This point of view is strengthened by some quantitative similarities. Let $\left\{\eta_{i}\right\}_{i \in I}$ be a centered Gaussian process, and let $d(i, j)$ be the natural metric on $I$ from (5). The following two lemmas are central to the proof of the majorizing measures theorem (Theorem (MM)). We refer to [35] [50] for their utility in the majorizing measures theory. The next lemma follows directly from the definition of the Gaussian density; see, for instance, [42, Lem. 5.1.3, Eq. (5.18)].

Lemma 1.10 (Gaussian concentration). For every $i, j \in I$, and $\alpha>0$,

$$
\mathbb{P}\left(\eta_{i}-\eta_{j}>\alpha\right) \leqslant \exp \left(\frac{-\alpha^{2}}{2 d(i, j)^{2}}\right) .
$$

The next result can be found in [35, Thm. 3.18].

Lemma 1.11 (Sudakov minoration). For every $\alpha>0$, If $I^{\prime} \subseteq I$ is such that $i, j \in I^{\prime}$ and $i \neq j$ implies $d(i, j) \geqslant \alpha$, then

$$
\mathbb{E} \sup _{i \in I^{\prime}} \eta_{i} \gtrsim \alpha \sqrt{\log \left|I^{\prime}\right|}
$$

Now, let $G=(V, E)$ be a network, and consider the associated continuous-time random walk $\left\{X_{t}\right\}$ with local times $L_{t}^{v}$. We define also the inverse local times $\tau^{v}(t)=\inf \left\{s: L_{s}^{v}>t\right\}$. An analog of the following lemma was proved in [30] for the discrete-time chain; the continuous-time version can be similarly proved, though we will not do so here, as it will not be used in the arguments to come. In interpreting the next lemma, it helps to recall that $L_{\tau^{u}(t)}^{u}=t$.

Lemma 1.12 (Concentration for local times). For all $u, v \in V$ and any $\alpha>0$ and $t \geqslant 0$, we have

$$
\mathbb{P}_{u}\left(L_{\tau^{u}(t)}^{u}-L_{\tau^{u}(t)}^{v} \geqslant \alpha\right) \leqslant \exp \left(\frac{-\alpha^{2}}{4 t R_{\mathrm{eff}}(u, v)}\right)
$$

where $\mathbb{P}_{u}$ denotes the measure for the random walk started at $u$.

Thus local times satisfy sub-gaussian concentration, where now the distance $d$ is replaced by $\sqrt{t \cdot R_{\text {eff }}}$. On the other side, the classical bound of Matthews [43] provides an analog to Lemma 1.11,

Lemma 1.13 (Matthews bound). For every $\alpha>0$, if $V^{\prime} \subseteq V$ is such that $u, v \in V^{\prime}$ and $u \neq v$ implies $H(u, v) \geqslant \alpha$, then

$$
t_{\mathrm{cov}}(G) \geqslant \alpha \log \left(\left|V^{\prime}\right|-1\right)
$$

Of course the similar structure of these lemmas offers no formal connection, but merely a hint that something deeper may be happening. We now discuss a far more concrete connection between local times and Gaussian processes.

The isomorphism theorems. The distribution of the local times for a Borel right process can be fully characterized by certain associated Gaussian processes; results of this flavor go by the name of Isomorphism Theorems. Several versions have been developed by Ray [44] and Knight [33], Dynkin [18, 17], Marcus and Rosen [40, 41], Eisenbaum [19] and Eisenbaum, Kaspi, Marcus, Rosen and Shi [20]. In what follows, we present the second Ray-Knight theorem in the special case of a continuous-time random walk. It first appeared in [20]; see also Theorem 8.2.2 of the book by Marcus and Rosen [42] (which contains a wealth of information on the connection between local times and Gaussian processes). It is easy to verify that the continuous-time random walk on a connected graph is indeed a recurrent strongly symmetric Borel right process. 
Theorem 1.14 (Generalized Second Ray-Knight Isomorphism Theorem). Fix $v_{0} \in V$ and define the inverse local time,

$$
\tau(t)=\inf \left\{s: L_{s}^{v_{0}}>t\right\} .
$$

Let $T_{0}$ be the hitting time to $v_{0}$ and let $\Gamma_{v_{0}}(x, y)=\mathbb{E}_{x}\left(L_{T_{0}}^{y}\right)$. Denote by $\eta=\left\{\eta_{x}: x \in V\right\}$ a mean zero Gaussian process with covariance $\Gamma_{v_{0}}(x, y)$. Let $P_{v_{0}}$ and $P_{\eta}$ be the measures on the processes $\left\{L_{T_{0}}^{x}\right\}$ and $\left\{\eta_{x}\right\}$, respectively. Then under the measure $P_{v_{0}} \times P_{\eta}$, for any $t>0$

$$
\left\{L_{\tau(t)}^{x}+\frac{1}{2} \eta_{x}^{2}: x \in V\right\} \stackrel{\text { law }}{=}\left\{\frac{1}{2}\left(\eta_{x}+\sqrt{2 t}\right)^{2}: x \in V\right\} .
$$

Thus to every continuous-time random walk, we can associate a Gaussian process $\left\{\eta_{v}\right\}_{v \in V}$. As discussed in Section 2.4, we have the relationship $d(u, v)=\sqrt{R_{\text {eff }}(u, v)}$, where $d(u, v)=$ $\sqrt{\mathbb{E}\left|\eta_{u}-\eta_{v}\right|^{2}}$. In particular, the process $\left\{\eta_{v}\right\}_{v \in V}$ is the Gaussian free field on the network $G$.

Using the Isomorphism Theorem in conjunction with concentration bounds for Gaussian processes, we already have enough machinery to prove the following upper bound in Section 2.1,

$$
t_{\mathrm{cov}}(G) \leqslant t_{\mathrm{bl}}(G, \delta) \lesssim \delta \mathcal{C}\left[\gamma_{2}(V, d)\right]^{2}=\mathcal{C}\left[\gamma_{2}\left(V, \sqrt{R_{\mathrm{eff}}}\right)\right]^{2}
$$

We also show how to prove a matching lower bound in terms of $\gamma_{2}$, but for a slightly different notion of "blanket time."

Thus (16) proves the first half of Theorem 1.9. The lower bound for cover times quite a bit more difficult to prove. Of course, the cover and return time relates to the event $\left\{\exists v: L_{\tau(t)}^{v}=0\right\}$, and unfortunately the correspondence (15) seems too coarse to provide lower bounds on the probability of this event directly.

To this end, we need to show that for the right value of $t$ in Theorem 1.14, we often have $\eta_{x} \approx-\sqrt{2 t}$ for some $x \in V$. The main difficulty is that we will have to show that there is often a vertex $x \in V$ with $\left|\eta_{x}+\sqrt{2 t}\right|$ being much smaller than the standard deviation of $\eta_{x}$. In doing so, we will use the full power of the majorizing measures theory, as well as the special structure of the Gaussian processes arising from the Isomorphism Theorem.

The discrete Gaussian free field and a tree-like subprocess. In Section 2.4 (see (35)), we recall that the Gaussian processes arising from the Isomorphism Theorem are not arbitrary, but correspond to the Gaussian free field (GFF) associated with $G$. Special properties of such processes will be essential to our proof of Theorem 1.9. In particular, if we use $R_{\text {eff }}(v, S)$ to denote the effective resistance between a point $v$ and a set of vertices $S \subseteq V$, then we have the relationship

$$
\sqrt{R_{\mathrm{eff}}(v, S)}=\operatorname{dist}_{L^{2}}\left(\eta_{v}, \operatorname{aff}\left(\left\{\eta_{w}\right\}_{w \in S}\right)\right),
$$

where aff $(\cdot)$ denotes the affine hull, and dist $_{L^{2}}$ is the $L^{2}$ distance in the Hilbert space underlying the process $\left\{\eta_{v}\right\}_{v \in V}$. In Section 2.3, we prove a number of properties of the effective resistance metric (e.g. Foster's network theorem); combined with (17), this yields some properties unique to processes arising from a GFF.

Next, in Section 3, we recall that one of the primary components of the majorizing measures theory is that every Gaussian process $\left\{\eta_{i}\right\}_{i \in I}$ contains a "tree like" subprocess which controls $\mathbb{E} \sup _{i \in I} \eta_{i}$. After a preprocessing step that ensures our trees have a number of additional features, 
we use the structure of the GFF to select a representative subtree with very strong independence properties that will be essential to our analysis of cover times.

Restructuring the randomness and a percolation argument. The majorizing measures theory is designed to control the first moment $\mathbb{E} \sup _{i \in I} \eta_{i}$ of the supremum of Gaussian process. In analyzing (15) to prove a lower bound on the cover times, we actually need to employ a variant of the second moment method. The need for this, and a detailed discussion of how it proceeds, are presented at the beginning of Section 4.

Towards this end, we want to associate events to the leaves of our "tree like" subprocess which can be thought of as "open events" in a percolation process on the tree. For general trees, it is known that the second moment method gives accurate estimates for the probability of having an open path to a leaf [38]. While our trees are not regular, they are "regularized" by the majorizing measure, and we do a somewhat standard analysis of such a process in Section 4.3.

The real difficulty involves setting up the right filtration on the probability space corresponding to our tree so that the percolation argument yields the desired control on the cover times. This requires a delicate definition of the events associated to each edge, and the ensuing analysis forms the technical core of our argument in Section 4 .

Algorithmic issues. In order to complete the proof of Theorem 1.5 and thus resolve Question 1.2. we present a deterministic algorithm which computes an approximation to $\gamma_{2}(X, d)$ for any metric space $(X, d)$. This is achieved in Section 3.3. While the algorithm is fairly elementary to describe, its analysis requires a number of tools from the majorizing measures theory.

We remark that, in combination with Theorem 1.9, this yields the following result.

Theorem 1.15. For any finite-state, reversible Markov chain presented as a network $G=(V, E)$ with given conductances $\left\{c_{x y}\right\}$, there is a deterministic, polynomial-time algorithm which computes a value $A(G)$ such that

$$
A(G) \asymp t_{\mathrm{cov}}(G) .
$$

Observe that for general reversible chains, the cover time is not necessarily bounded a polynomial in $|V|$, and thus even randomized simulation of the chain does not yield a polynomial-time algorithm for approximating $t_{\mathrm{cov}}(G)$. Finally, in Section 4.5, we prove Theorems 1.7 and 1.8 in the setting of arbitrary reversible Markov chains, leading to a near-linear time randomized algorithm for computing cover times.

\section{Gaussian processes and local times}

We now discuss properties of the Gaussian processes arising from the isomorphism theorem (Theorem 1.14). In Section 2.1, we show that the isomorphism theorem, combined with concentration properties of Gaussian processes, is already enough to get strong control on blanket times and related quantities.

In Section 2.3, we prove some geometric properties of the resistance metric on networks that will be crucial to our work on the cover time in Sections 3 and 4 . Finally, in Section 2.4, we recall the definition of the Gaussian free field and show how the geometry of such a process relates to the geometry of the underlying resistance metric. 


\subsection{The blanket time}

We first remark that the covariance matrix of the Gaussian process arising from the isomorphism theorem can be calculated explicitly in terms of the resistance metric on the network $G(V)$. Throughout this section, the process $\left\{\eta_{x}\right\}_{x \in V}$ refers to the one resulting from Theorem 1.14 with $v_{0} \in V$ some fixed (but arbitrary) vertex, $\tau(t)$ refers to the inverse local time defined in (14), and $T_{0}$ is the hitting time to $v_{0}$.

Lemma 2.1. For every $x, y \in V$,

$$
\Gamma_{v_{0}}(x, y)=\mathbb{E}_{x}\left(L_{T_{0}}^{y}\right)=\frac{1}{2}\left(R_{\mathrm{eff}}\left(x, v_{0}\right)+R_{\mathrm{eff}}\left(v_{0}, y\right)-\mathrm{R}_{\mathrm{eff}}(x, y)\right) .
$$

In particular,

$$
\mathbb{E}\left(\eta_{x}-\eta_{y}\right)^{2}=R_{\mathrm{eff}}(x, y)
$$

Proof. To prove the lemma, we use the cycle identity for hitting times (see, e.g., [36, Lem. 10.10]) which asserts that,

$$
H\left(x, v_{0}\right)+H\left(v_{0}, y\right)+H(y, x)=H(x, y)+H\left(y, v_{0}\right)+H\left(v_{0}, x\right) .
$$

Averaging both sides of (18) and recalling (9) yields

$$
H\left(x, v_{0}\right)+H\left(v_{0}, y\right)+H(y, x)=\frac{\mathcal{C}}{2}\left[R_{\mathrm{eff}}\left(x, v_{0}\right)+R_{\mathrm{eff}}\left(v_{0}, y\right)+R_{\mathrm{eff}}(x, y)\right]
$$

Now, we subtract $\mathcal{C} R_{\mathrm{eff}}(x, y)=H(x, y)+H(y, x)$ from both sides, giving

$$
H\left(x, v_{0}\right)+H\left(v_{0}, y\right)-H(x, y)=\frac{\mathcal{C}}{2}\left[R_{\mathrm{eff}}\left(x, v_{0},\right)+R_{\mathrm{eff}}\left(v_{0}, y\right)-R_{\mathrm{eff}}(x, y)\right]
$$

Finally, we conclude using the identity (see, e.g. [2, Ch 2., Lem. 9]),

$$
\mathbb{E}_{x}\left(L_{T_{0}}^{y}\right)=\frac{1}{\mathcal{C}}\left(H\left(x, v_{0}\right)+H\left(v_{0}, y\right)-H(x, y)\right) .
$$

We now relate the blanket time of the random walk to the expected supremum of its associated Gaussian process. The following is a central facet of the theory of concentration of measure; see, for example, [34, Thm. 7.1, Eq. (7.4)].

Lemma 2.2. Consider a Gaussian process $\left\{\eta_{x}: x \in V\right\}$ and define $\sigma=\sup _{x \in V}\left(\mathbb{E}\left(\eta_{x}^{2}\right)\right)^{1 / 2}$. Then for $\alpha>0$,

$$
\mathbb{P}\left(\left|\sup _{x \in V} \eta_{x}-\mathbb{E} \sup _{x \in V} \eta_{x}\right|>\alpha\right) \leqslant 2 \exp \left(-\alpha^{2} / 2 \sigma^{2}\right)
$$

We are now ready to establish the upper bound on the strong blanket time $t_{\mathrm{bl}}^{\star}(G, \delta)$, for any fixed $0<\delta<1$. Note that this will naturally yield an upper bound on $t_{\mathrm{bl}}(\delta)$.

Theorem 2.3. Consider a network $G(V)$ and its total conductance $\mathcal{C}=\sum_{x \in V} c_{x}$. For any fixed $0<\delta<1$, the blanket time $t_{\mathrm{bl}}^{\star}(G, \delta)$ of the random walk on $G(V)$ satisfies

$$
t_{\mathrm{bl}}^{\star}(G, \delta) \lesssim_{\delta} \mathcal{C} \cdot\left(\mathbb{E} \sup _{x \in V} \eta_{x}\right)^{2},
$$

where $\left\{\eta_{x}\right\}$ is the associated Gaussian process from Theorem 1.14 . 
Proof. We first prove that for some $A_{\delta}>0$

$$
t_{\mathrm{bl}}^{\star}(\delta) \leqslant A_{\delta} \mathcal{C}\left(\left(\mathbb{E} \sup _{x \in V} \eta_{x}\right)^{2}+\sup _{x \in V} \mathbb{E}\left(\eta_{x}^{2}\right)\right) .
$$

Fix a vertex $v_{0} \in V$ and consider the local times $\left\{L_{\tau(t)}^{x}: x \in V\right\}$, where for $t>0$, we write $\tau(t)=\inf \left\{s: L_{s}^{v_{0}}>t\right\}$. Let $\sigma=\sup _{x \in V} \sqrt{\mathbb{E}\left(\eta_{x}^{2}\right)}$ and $\Lambda=\mathbb{E} \sup _{x} \eta_{x}$.

Use $\left\{\eta_{x}^{L}\right\}$ to denote the copy of the Gaussian process corresponding to the left-hand side of (15), and $\left\{\eta_{x}^{R}\right\}$ to denote the i.i.d. process corresponding to the right-hand side. Fix $\beta>0$, and set $t=t(\beta)=\beta\left(\Lambda^{2}+\sigma^{2}\right)$. By Theorem 1.14, we get that

$$
\mathbb{P}\left(\min _{x} L_{\tau(t)}^{x} \leqslant \sqrt{\delta} t\right) \leqslant \mathbb{P}\left(\inf _{x} \frac{1}{2}\left(\eta_{x}^{R}+\sqrt{2 t}\right)^{2} \leqslant \frac{1+\sqrt{\delta}}{2} t\right)+\mathbb{P}\left(\sup _{x} \frac{1}{2}\left(\eta_{x}^{L}\right)^{2} \geqslant \frac{1-\sqrt{\delta}}{2} t\right) .
$$

Therefore,

$$
\mathbb{P}\left(\min _{x} L_{\tau(t)}^{x} \leqslant \sqrt{\delta} t\right) \leqslant \mathbb{P}\left(\inf _{x} \eta_{x}^{R} \leqslant-a_{\delta} \sqrt{t}\right)+\mathbb{P}\left(\sup _{x}\left|\eta_{x}^{L}\right| \geqslant b_{\delta} \sqrt{t}\right)
$$

where $a_{\delta}=\sqrt{2}-\sqrt{1+\sqrt{\delta}}$ and $b_{\delta}=\sqrt{1-\sqrt{\delta}}$. Applying Lemma 2.2, we obtain that if $\beta>\beta_{0}(\delta)$ for some $\beta_{0}(\delta)>0$, then

$$
\mathbb{P}\left(\min _{x} L_{\tau(t)}^{x} \leqslant \sqrt{\delta} t\right) \leqslant 6 \exp \left(-\gamma_{\delta} \beta\right),
$$

where $\gamma_{\delta}=\frac{1}{2}\left(a_{\delta}^{2} \wedge b_{\delta}^{2}\right)$. On the other hand, we have

$$
\mathbb{P}\left(\max _{x} L_{\tau(t)}^{x} \geqslant t / \sqrt{\delta}\right) \leqslant \mathbb{P}\left(\max _{x} \frac{1}{2}\left(\eta_{x}^{R}+\sqrt{2 t}\right)^{2} \geqslant t / \sqrt{\delta}\right)=\mathbb{P}\left(\max _{x} \eta_{x} \geqslant a_{\delta}^{\prime} \sqrt{t}\right)
$$

where $a_{\delta}^{\prime}=\sqrt{1 / \delta}-1$. Applying Lemma 2.2 again for $\beta>\beta_{0}(\delta)$, we get that

$$
\mathbb{P}\left(\max _{x} L_{\tau(t)}^{x} \geqslant t / \sqrt{\delta}\right) \leqslant 2 \exp \left(-\gamma_{\delta}^{\prime} \beta\right)
$$

where $\gamma_{\delta}^{\prime}=\left(a_{\delta}^{\prime}\right)^{2} / 2$. Note that assuming $\min _{x} L_{\tau(t)}^{x} \geqslant \sqrt{\delta} t$ and $\max _{x} L_{\tau(t)}^{x} \leqslant t / \sqrt{\delta}$, we have $\tau(t)=$ $\sum_{x} c_{x} L_{\tau(t)}^{x} \leqslant \mathcal{C} t / \sqrt{\delta}$ as well as $\min _{x, y} L_{\tau(t)}^{x} / L_{\tau(t)}^{y} \geqslant \delta$. It then follows that $\tau_{\mathrm{bl}}^{\star} \leqslant \tau(t) \leqslant \mathcal{C} t / \sqrt{\delta}$. Therefore, we can deduce that

$$
\left\{\tau_{\mathrm{bl}}^{\star} \geqslant \mathcal{C} t / \sqrt{\delta}\right\} \subset\left\{\min _{x} L_{\tau(t)}^{x} \leqslant \sqrt{\delta} t\right\} \bigcup\left\{\max _{x} L_{\tau(t)}^{x} \geqslant t / \sqrt{\delta}\right\} .
$$

Combined with (20) and (21), it yields that

$$
\mathbb{P}\left(\tau_{\mathrm{bl}}^{\star} \geqslant \mathcal{C} t / \sqrt{\delta}\right) \leqslant 6 \exp \left(-\gamma_{\delta} \beta\right)+2 \exp \left(-\gamma_{\delta}^{\prime} \beta\right) .
$$

It then follows that $t_{\mathrm{bl}}^{\star} \leqslant A_{\delta} \mathcal{C}\left(\Lambda^{2}+\sigma^{2}\right)$ for some $A_{\delta}>0$ which depends only on $\delta$, establishing (19).

It remains to prove that $\sigma=O(\Lambda)$. To this end, let $x^{*}$ be such that $\mathbb{E} \eta_{x^{*}}^{2}=\sigma^{2}$. We have

$$
\Lambda \geqslant \mathbb{E} \max \left(\eta_{v_{0}}, \eta_{x^{*}}\right)=\mathbb{E} \max \left(0, \eta_{x^{*}}\right)=\frac{\sigma}{\sqrt{2 \pi}} .
$$

This completes the proof for the continuous-time case. 
Remark 1. An interesting question is the asymptotic behavior of $\delta$-blanket time as $\delta \rightarrow 1$, namely the dependence on $\delta$ of $A_{\delta}$ in (19). As implied in the proof, we can see that

$$
A_{\delta} \lesssim \frac{1}{\gamma_{\delta}}+\frac{1}{\gamma_{\delta}^{\prime}} \lesssim \frac{1}{(1-\delta)^{2}}
$$

These asymptotics are tight for the complete graph; see e.g. [54, Cor. 2].

We next extend the proof of the preceding theorem to the case of the discrete-time random walk. The next lemma contains the main estimate required for this extension.

Lemma 2.4. Let $G(V)$ be a network and write $\gamma_{2}=\gamma_{2}\left(V, \sqrt{R_{\mathrm{eff}}}\right)$. Then for all $u \geqslant 16$, we have

$$
\sum_{v \in V} e^{-u \cdot c_{v} \gamma_{2}^{2}} \lesssim e^{-u / 8}
$$

Proof. By definition of the $\gamma_{2}$ functional, we can choose a sequence of partitions $\mathcal{A}_{k}$ with $\left|\mathcal{A}_{k}\right| \leqslant 2^{2^{k}}$ such that

$$
\gamma_{2} \geqslant \frac{1}{2} \sup _{v \in V} \sum_{k \geqslant 0} 2^{k / 2} \operatorname{diam}\left(A_{k}(v)\right) .
$$

For $v \in V$, let $k_{v}=\min \left\{k:\{v\} \in \mathcal{A}_{k}\right\}$. It is clear that $R_{\text {eff }}(u, v) \geqslant 1 / c_{v}$ for all $u \neq v$ and hence $\left(\operatorname{diam}\left(A_{k_{v}-1}(v)\right)\right)^{2} \geqslant 1 / c_{v}$. Therefore, we see that

$$
\sum_{v \in V} e^{-u \cdot c_{v} \gamma_{2}^{2}}=\sum_{k=0}^{\infty} \sum_{v: k_{v}=k+1} e^{-u \cdot c_{v} \gamma_{2}^{2}} \leqslant \sum_{k=1}^{\infty} 2^{2^{k+1}} e^{-u 2^{k} / 4} \lesssim e^{-u / 8},
$$

completing the proof.

Theorem 2.5. Consider a network $G(V)$ and its total conductance $\mathcal{C}=\sum_{x \in V} c_{x}$. For any fixed $0<\delta<1$, the discrete blanket time $t_{\mathrm{bl}}(G, \delta)$ of the random walk on on $G(V)$ satisfies

$$
t_{\mathrm{bl}}(G, \delta) \lesssim \delta \mathcal{C} \cdot\left(\mathbb{E} \sup _{x \in V} \eta_{x}\right)^{2}
$$

where $\left\{\eta_{x}\right\}$ is the associated Gaussian process from Theorem 1.14.

Proof. We now consider the embedded discrete-time random walk of the continuous-time counterpart (i.e. the corresponding jump chain; see [2, Ch. 2]). Let $N_{t}^{v}$ be such that $c_{v} \cdot N_{t}^{v}$ is the number of visits to vertex $v$ up to continuous time $t$, i.e. $N_{t}^{v}$ is a discrete-time analog of the local time $L_{t}^{v}$.

Fix a vertex $v_{0} \in V$ and consider the local times $\left\{L_{\tau(t)}^{x}: x \in V\right\}$. Let $\sigma=\sup _{x \in V} \sqrt{\mathbb{E}\left(\eta_{x}^{2}\right)}$ and $\Lambda=\mathbb{E} \sup _{x} \eta_{x}$. Again, set $t=\beta\left(\Lambda^{2}+\sigma^{2}\right)$.

Let $\tau_{\mathrm{bl}}(\delta)$ denote the first time at which $N_{t}^{x} \geqslant \frac{\delta t}{\mathcal{C}}$ for every $x \in V$. Assuming that $\min _{x} N_{\tau(t)}^{x} \geqslant$ $\delta^{1 / 4} t$ and $\max _{x} N_{\tau(t)}^{x} \leqslant t / \delta^{3 / 4}$, we have $\tau(t)=\sum_{x} c_{x} N_{\tau(t)}^{x} \leqslant \mathcal{C} t / \delta^{3 / 4}$ and thus $\min _{x} N_{\tau(t)}^{x} \geqslant \delta \tau(t) / \mathcal{C}$. It then follows that $\tau_{\mathrm{bl}}(\delta) \leqslant \tau(t) \leqslant \mathcal{C} t / \delta^{3 / 4}$. Therefore, we deduce that

$$
\left\{\tau_{\mathrm{bl}}(\delta) \geqslant \frac{\mathcal{C} t}{\delta^{3 / 4}}\right\} \subset\left\{\min _{x} N_{\tau(t)}^{x} \leqslant \delta^{1 / 4} t\right\} \bigcup\left\{\max _{x} N_{\tau(t)}^{x} \geqslant t / \delta^{3 / 4}\right\}
$$


Therefore we have,

$$
\begin{aligned}
\mathbb{P}\left(\tau_{\mathrm{bl}}(\delta) \geqslant \frac{\mathcal{C} t}{\delta^{3 / 4}}\right) & \leqslant \mathbb{P}\left(\min _{x} L_{\tau(t)}^{x} \leqslant \sqrt{\delta} t \text { or } \max _{x} L_{\tau(t)}^{x} \geqslant t / \sqrt{\delta}\right) \\
& +\mathbb{P}\left(\forall x: \sqrt{\delta} t \leqslant L_{\tau(t)}^{x} \leqslant t / \sqrt{\delta} \mid \min _{x} N_{\tau(t)}^{x} \leqslant \delta^{1 / 4} t \text { or } \max _{x} N_{\tau(t)}^{x} \geqslant t / \delta^{3 / 4}\right) .
\end{aligned}
$$

Note that we have already bounded the first term in (20) and (21).

The second term can be bounded by a simple application of a large deviation inequality on the sum of i.i.d. exponential variables. Precisely,

$$
\sum_{x \in V} \mathbb{P}\left(\sqrt{\delta} t \leqslant L_{\tau(t)}^{x} \leqslant t / \sqrt{\delta} \mid N_{\tau(t)}^{x} \leqslant \delta^{1 / 4} t \text { or } N_{\tau(t)}^{x} \geqslant t / \delta^{3 / 4}\right) \lesssim \sum_{x \in V} e^{-\tilde{a}_{\delta} \cdot c_{x} t}
$$

for some constant $\tilde{a}_{\delta}>0$ depending only on $\delta$. Recall that Theorem (MM) implies $\mathbb{E} \sup _{x} \eta_{x} \asymp$ $\gamma_{2}\left(V, \sqrt{R_{\text {eff }}}\right)$. By (22) , we see that $\sigma \leqslant \sqrt{2 \pi} \Lambda$. Altogether, we get that $t \asymp \Lambda^{2} \asymp \beta\left[\gamma_{2}\left(V, \sqrt{R_{\text {eff }}}\right)\right]^{2}$. Applying Lemma 2.4, we conclude that there exists $\tilde{\beta}_{0}(\delta)>0$ depending only on $\delta$ such that for all $\beta \geqslant \tilde{\beta}_{0}(\delta)$, we have

$$
\mathbb{P}\left(\tau_{\mathrm{bl}}(G, \delta) \geqslant \mathcal{C} t / \delta^{3 / 4}\right) \lesssim e^{-\tilde{b}_{\delta} \beta}
$$

where $\tilde{b}_{\delta}$ is a constant depending only on $\delta$. This immediately yields the desired upper bound on the blanket time for the discrete-time random walk.

We next exhibit a lower bound on a variation of blanket time (considered in [30]). It is apparent that the lower bound on the cover time, which will be proved in Section 4, is an automatic lower bound on the blanket time. In what follows, though, we try to give a simple argument that can be regarded as a warm up. For the convenience of analysis, we consider the following notion. For $0<\varepsilon<1$, define

$$
t_{\mathrm{bl}}^{*}(G, \varepsilon)=\max _{w \in V} \inf \left\{s: \mathbb{P}_{w}\left(\forall u, v \in V: L_{t}^{u} \leqslant 2 L_{t}^{v}\right)>\varepsilon \text { for all } t \geqslant s\right\} .
$$

Theorem 2.6. Consider a network $G(V)$ and its total conductance $\mathcal{C}=\sum_{x \in V} c_{x}$. For any fixed $0<\varepsilon<1$, we have

$$
t_{\mathrm{bl}}^{*}(G, \varepsilon) \gtrsim \varepsilon \mathcal{C} \cdot\left(\mathbb{E} \sup _{x \in V} \eta_{x}\right)^{2}
$$

In order to prove Theorem 2.6. we will use the next simple lemma. We will also require this estimate in Section 4 .

Lemma 2.7. Let $\tau(t)$ be the inverse local time at vertex $v_{0}$, as defined in (14). Let $\mathcal{C}$ be the total conductance and let $\mathfrak{D}=\max _{x, y \in V} \sqrt{R_{\mathrm{eff}}(x, y)}$. Then, for all $\beta>0$ and $t \geqslant \mathfrak{D}^{2} / \beta^{2}$,

$$
\mathbb{P}_{v_{0}}(\tau(t) \leqslant \beta \mathcal{C} t) \leqslant 3 \beta .
$$

Proof. We use $\mathbb{P}_{v}$ to denote the measure on random walks started at a vertex $v \in V$, and we use $\mathbb{E}_{v}$ similarly. Let $p_{\delta}=\min _{v}\left\{\mathbb{P}_{v}(\tau(t) \leqslant \delta \mathcal{C} t)\right\}$ for some $\delta>0$. Using the strong Markov property, we get that for all $v \in V$,

$$
\mathbb{P}_{v}(\tau(t) \geqslant k \delta \mathcal{C} t) \leqslant\left(1-p_{\delta}\right)^{k} .
$$


In particular, $\mathbb{E}_{v} \tau(t) \leqslant \delta \mathcal{C} t / p_{\delta}$.

By Theorem 1.14, it follows easily that $\mathbb{E}_{v_{0}} \tau(t)=\mathcal{C} t$. Since $\mathbb{E}_{v} \tau(t) \geqslant \mathbb{E}_{v_{0}}(\tau(t))$, we deduce that $p_{\delta} \leqslant \delta$. Let $u=u(\delta)$ be such that $\mathbb{P}_{u}(\tau(t) \leqslant \delta \mathcal{C} t)=p_{\delta}$. Let $Y, Z$ be random variables with the law $\tau(t)$, when the random walk is started at $u$ and $v_{0}$, respectively. Clearly,

$$
Y \stackrel{\text { law }}{=} Z+T_{v_{0}}
$$

where $T_{v_{0}}$ is distributed as the hitting time to $v_{0}$, when then random walk is started at $u$ and $T_{v_{0}}$ is independent of $Z$.

Since $R_{\text {eff }}\left(u, v_{0}\right) \leqslant \mathfrak{D}^{2}$, we have $\mathbb{E}_{u} T_{v_{0}} \leqslant \mathcal{C} \mathfrak{D}^{2}$ (by (9) ), and this yields $\mathbb{P}_{u}\left(T_{v_{0}} \geqslant \mathcal{C} \mathfrak{D}^{2} / \beta\right) \leqslant \beta$. Using the assumption $t \geqslant \mathfrak{D}^{2} / \beta^{2}$ and (24), we conclude that

$$
\mathbb{P}(Z \leqslant \beta \mathcal{C} t) \leqslant \mathbb{P}\left(Z \leqslant 2 \beta \mathcal{C} t-\mathcal{C} \mathfrak{D}^{2} / \beta\right) \leqslant \mathbb{P}(Y \leqslant 2 \beta \mathcal{C} t)+\mathbb{P}\left(T_{v_{0}} \geqslant \mathcal{C} \mathfrak{D}^{2} / \beta\right) \leqslant p_{2 \beta}+\beta \leqslant 3 \beta,
$$

as required.

We are now ready to establish the lower bound on $t_{\mathrm{bl}}^{*}(G, \varepsilon)$.

Proof of Theorem 2.6. We consider the associated Gaussian process as in the proof of Theorem 2.3 , Let $\sigma=\sup _{x \in V} \sqrt{\mathbb{E} \eta_{x}^{2}}$ and $\Lambda=\mathbb{E} \sup _{x} \eta_{x}$. Observe that the maximal hitting time is a simple lower bound on $t_{\mathrm{bl}}^{*}(G, \varepsilon)$ up to a constant depending only on $\varepsilon$. In light of Lemma 2.1, we see $t_{\mathrm{bl}}^{*}(G, \varepsilon) \gtrsim_{\varepsilon} \mathcal{C} \cdot \sigma^{2}$. Therefore, we can assume in what follows

$$
\Lambda^{2} \geqslant 100 \log (4 / \varepsilon) \varepsilon^{-2} \sigma^{2}
$$

Let $t_{*}=\frac{1}{2} \Lambda^{2}$. By Lemma 2.2 , we get

$$
\mathbb{P}\left(\inf _{x \in V} \frac{1}{2}\left(\eta_{x}^{R}+\sqrt{2 t_{*}}\right)^{2} \leqslant \log (4 / \varepsilon) \sigma^{2}\right) \geqslant \mathbb{P}\left(\left|\sup _{x \in V} \eta_{x}^{R}-\Lambda\right| \leqslant \sqrt{2 \log (4 / \varepsilon)} \sigma\right) \geqslant 1-\frac{\varepsilon}{2}
$$

Applying Theorem 1.14, we obtain

$$
\mathbb{P}\left(\inf _{x \in V} L_{\tau\left(t_{*}\right)}^{x} \leqslant \log (4 / \varepsilon) \sigma^{2}\right) \geqslant 1-\frac{\varepsilon}{2}
$$

By triangle inequality, we have $\mathfrak{D} \leqslant 2 \sigma$. Recalling the assumption (25), we can apply Lemma 2.7 and deduce that

$$
\mathbb{P}\left(\tau\left(t_{*}\right) \leqslant \varepsilon \mathcal{C} t_{*} / 6\right) \leqslant \varepsilon / 2 .
$$

Writing $t_{0}=\varepsilon \mathcal{C} t_{*} / 6$, we can then obtain that

$$
\mathbb{P}\left(\inf _{x \in V} L_{t_{0}}^{x} \leqslant \log (4 / \varepsilon) \sigma^{2}, \tau\left(t_{*}\right) \geqslant t_{0}\right) \geqslant 1-\varepsilon
$$

Also, we see that $\sup _{x \in V} L_{t_{0}}^{x} \geqslant \varepsilon \Lambda^{2} / 12$ whenever $\tau\left(t_{*}\right) \geqslant t_{0}$. Using assumption (25) again, we conclude

$$
\mathbb{P}_{v_{0}}\left(\exists x, y \in V: L_{t_{0}}^{x}>2 L_{t_{0}}^{y}\right) \geqslant 1-\varepsilon .
$$

This implies that $t_{\mathrm{bl}}^{*}(G, \varepsilon) \geqslant t_{0}$, completing the proof. 


\subsection{An asymptotically strong upper bound}

Finally, we show a strong upper bound for the asymptotics of $t_{\text {cov }}$ on a sequence of graphs $\left\{G_{n}\right\}$, assuming $t_{\text {hit }}\left(G_{n}\right)=o\left(t_{\operatorname{cov}}\left(G_{n}\right)\right)$.

Theorem 2.8. For any graph $G=(V, E)$ with $v_{0} \in V$, let $t_{\text {hit }}(G)$ be the maximal hitting time in $G$ and let $\left\{\eta_{v}\right\}_{v \in V}$ be the GFF on $G$ with $\eta_{v_{0}}=0$. Then, for a universal constant $C>0$,

$$
t_{\mathrm{cov}}(G) \leqslant\left(1+C \sqrt{\frac{t_{\mathrm{hit}}(G)}{t_{\mathrm{cov}}(G)}}\right) \cdot|E| \cdot\left(\mathbb{E} \sup _{v \in V} \eta_{v}\right)^{2} .
$$

Proof. Theorem 2.5 asserts that

$$
t_{\mathrm{cov}}(G) \preceq\left(\mathbb{E} \max _{v} \eta_{v}\right)^{2}
$$

where $\preceq$ denotes stochastic domination. Write $\sigma^{2}=\max _{v} \mathbb{E} \eta_{v}^{2}$. Note that $\sigma^{2}$ corresponds to the diameter of $V$ in the effective resistance metric, thus $t_{\text {hit }}(G) \asymp|E| \sigma^{2}$. Denote by $S=\sum_{v} d_{v} \eta_{v}^{2}$, where $d_{v}$ is the degree of vertex $v$. By a generalized Hölder inequality and moment estimates for Gaussian variables (here we use that $\mathbb{E} X^{6}=15$ for a standard Gaussian variable $X$ ), we obtain that

$$
\mathbb{E} S^{3} \leqslant \sum_{u, v, w} d_{u} d_{v} d_{w} \mathbb{E}\left(\eta_{u}^{2} \eta_{v}^{2} \eta_{w}^{2}\right) \leqslant \sum_{u, v, w} d_{u} d_{v} d_{w} \mathbb{E}\left(\eta_{u}^{6}\right)^{1 / 3} \mathbb{E}\left(\eta_{v}^{6}\right)^{1 / 3} \mathbb{E}\left(\eta_{w}^{6}\right)^{1 / 3} \leqslant 15|E|^{3} \sigma^{6}
$$

An application of Markov's inequality then yields

$$
\mathbb{P}\left(S \geqslant \alpha|E| \sigma^{2}\right) \leqslant \frac{15}{\alpha^{3}} .
$$

Write $Q=\sum_{v} d_{v} \eta_{v}$. Clearly, $Q$ is a centered Gaussian with variance bounded by $4|E|^{2} \sigma^{2}$ and therefore,

$$
\mathbb{P}(|Q| \geqslant \alpha|E| \sigma) \leqslant 2 \mathrm{e}^{-\alpha^{2} / 8} .
$$

For $\beta>0$, let $t=\frac{1}{2}\left(\mathbb{E} \max _{v} \eta_{v}+\beta \sigma\right)^{2}$. Noting $\tau(t)=\sum_{v} d_{v} L_{\tau(t)}^{v}$ and recalling the Isomorphism theorem (Theorem 1.14), we get that

$$
\tau(t) \preceq 2|E| t+\frac{\sqrt{2 t}}{2}|Q|+\frac{1}{2} S .
$$

Combined with (27) and (28), we deduce that

$$
\mathbb{P}\left(\tau(t) \geqslant 2|E| t+\sqrt{2 t} \beta|E| \sigma+\beta|E| \sigma^{2}\right) \leqslant \frac{12}{(\beta-2)^{2}}+2 \mathrm{e}^{-\beta^{2} / 8} .
$$

We now turn to bound the probability for $\tau_{\text {cov }}>\tau(t)$. Observe that on the event $\left\{\tau_{\text {cov }}>\tau(t)\right\}$, there exists $v \in V$ such that $L_{\tau(t)}^{v}=0$. It is clear that for all $v \in V$, we have $\mathbb{P}\left(\eta_{v}^{2} \geqslant \beta \sigma^{2} / 2\right) \leqslant$ $2 \mathrm{e}^{-\beta / 4}$. Since $\left\{\eta_{v}\right\}_{v \in V}$ and $\left\{L_{\tau(t)}^{v}\right\}_{v \in V}$ are two independent processes, we obtain

$$
\mathbb{P}\left(\left\{\tau_{\text {cov }}>\tau(t)\right\} \backslash\left\{\exists v \in V: L_{\tau(t)}^{v}+\frac{1}{2} \eta_{v}^{2}<\beta \sigma^{2} / 2\right\}\right) \leqslant 2 \mathrm{e}^{-\beta / 4} .
$$


On the other hand, we deduce from the concentration of Gaussian processes (Lemma 2.2) that

$$
\mathbb{P}\left(\inf _{v}\left(\sqrt{2 t}+\eta_{v}\right)^{2} \leqslant \beta \sigma / 2\right) \leqslant 2 \mathrm{e}^{-\beta / 8} .
$$

Applying Isomorphism theorem again and combined with (30), we get that

$$
\mathbb{P}\left(\tau_{\text {cov }}>\tau(t)\right) \leqslant 4 \mathrm{e}^{-\beta / 8} .
$$

Combined with (29), it follows that

$$
\mathbb{P}\left(\tau_{\text {cov }} \geqslant 2|E| t+\sqrt{2 t} \beta|E| \sigma+\beta|E| \sigma^{2}\right) \leqslant \frac{15}{\beta^{3}}+2 \mathrm{e}^{-\beta^{2} / 8}+4 \mathrm{e}^{-\beta / 8} .
$$

Since $t=\frac{1}{2}\left(\mathbb{E} \max _{v} \eta_{v}+\beta \sigma\right)^{2}$, we can deduce that for some universal constant $C_{1}>0$,

$$
t_{\mathrm{cov}}(G) \leqslant|E|\left(\mathbb{E} \sup _{v} \eta_{v}\right)^{2}+C_{1}|E|\left(\sigma^{2}+\sigma \mathbb{E} \sup _{v} \eta_{v}\right) .
$$

Recalling (26), we complete the proof.

\subsection{Geometry of the resistance metric}

We now discuss some relevant properties of the resistance metric on a network $G(V)$.

Effective resistances and network reduction. For a subset $S \subseteq V$, define the quotient network $G / S$ to have vertex set $(V \backslash S) \cup\left\{v_{S}\right\}$, where $v_{S}$ is a new vertex disjoint from $V$. The conductances in $G / S$ are defined by $c_{x y}^{G / S}=c_{x y}$ if $x, y \notin S$ and $c_{v_{S} x}=\sum_{y \in S} c_{x y}$ for $x \notin S$.

Now, given $v \in V$ and $S \subseteq V$, we put

$$
R_{\mathrm{eff}}(v, S) \triangleq R_{\mathrm{eff}}^{G / S}\left(v, v_{S}\right)
$$

where the latter effective resistance is computed in $G / S$. For two disjoint sets $S, T \subseteq V$, we define

$$
R_{\mathrm{eff}}(S, T) \triangleq R_{\mathrm{eff}}^{G / S}\left(v_{S}, T\right)
$$

and the resistance is defined to be 0 if $S \cap T \neq \emptyset$. It is straightforward to check that $R_{\text {eff }}(S, T)=$ $R_{\text {eff }}(T, S)$. The following network reduction lemma was discovered by Campbell [10] under the name "star-mesh transformation" (see also, e.g., [39, Ex. 2.47(d)]). We give a proof for completeness.

Lemma 2.9. For a network $G(V)$ and a subset $\widetilde{V} \subset V$, there exists a network $\tilde{G}(\tilde{V})$ such that for all $u, v \in \widetilde{V}$, we have

$$
\tilde{c}_{v}=c_{v} \text { and } R_{\mathrm{eff}}^{\widetilde{G}}(u, v)=R_{\mathrm{eff}}(u, v) .
$$

We call $\widetilde{G}(\widetilde{V})$ the reduced network. Furthermore, if $\widetilde{V}=V \backslash\{x\}$, we then have the formula

$$
\tilde{c}_{y z}=c_{y z}+c_{y z}^{*, x}, \text { where } c_{y z}^{*, x}=\frac{c_{x y} c_{x z}}{\sum_{w \in V_{x}} c_{x w}} .
$$


Proof. Let $P$ be the transition kernel of the discrete-time random walk $\left\{S_{t}\right\}$ on the network $G$ and let $P^{\widetilde{V}}$ be the transition kernel of the induced random walk on $\widetilde{V}$, namely for $u, v \in \widetilde{V}$

$$
P^{\widetilde{V}}(u, v)=\mathbb{P}_{u}\left(T_{\widetilde{V}}^{+}=v\right),
$$

where $T_{A}^{+} \triangleq \min \left\{t \geqslant 1: S_{t} \in A\right\}$ for all $A \subseteq V$. In other words, $P^{\widetilde{V}}$ is the chain watched in the subset $\widetilde{V}$. We observe that $P^{\widetilde{V}}$ is a reversible Markov chain on $\widetilde{V}$ (see, e.g., [2, 36]). It is clear that the chain $P^{\widetilde{V}}$ has the same invariant measure as that of $P$ restricted to $\widetilde{V}$, up to scaling by a constant. Therefore, there exists a (unique) network $\widetilde{G}(\widetilde{V})$ corresponding to the Markov chain $P^{\widetilde{V}}$ such that $\tilde{c}_{u}=c_{u}$ for all $u \in \widetilde{V}$.

We next show that the effective resistances are preserved in $\widetilde{G}(\widetilde{V})$. To this end, we use the following identity relating effective resistance and the random walk (see, e.g., [39, Eq. (2.5)]),

$$
\mathbb{P}_{v}\left(T_{v}^{+}>T_{u}\right)=\frac{1}{c_{v} R_{\mathrm{eff}}(u, v)}
$$

where $T_{u}=\min \left\{t \geqslant 0: S_{t}=u\right\}$. Since $P^{\widetilde{V}}$ is a watched chain on the subset $\widetilde{V}$, we see that $\mathbb{P}_{v}^{\widetilde{V}}\left(T_{v}^{+}>T_{u}\right)=\mathbb{P}_{v}\left(T_{v}^{+}>T_{u}\right)$ for all $u, v \in \widetilde{V}$. This yields $R_{\mathrm{eff}}^{\widetilde{G}}(u, v)=R_{\mathrm{eff}}(u, v)$.

To prove the second half of the lemma, we let $\widetilde{G}(\widetilde{V})$ be the network defined by (32). A straightforward calculation yields that

$$
\tilde{c}_{v}=c_{v}-c_{x v}+\sum_{y \in V_{x}} c_{v y}^{*, x}=c_{v}-c_{x v}+\sum_{y \in V_{x}} \frac{c_{x v} c_{x y}}{\sum_{z \in V_{x}} c_{x z}}=c_{v} .
$$

Let $P^{\widetilde{G}}$ be the transition kernel for the random walk on the network $\widetilde{G}(\widetilde{V})$. Then,

$$
P^{\widetilde{G}}(u, v)=\frac{\tilde{c}_{u v}}{\tilde{c}_{u}}=\frac{c_{u v}+\frac{c_{u x} c_{x v}}{\sum_{y \in V_{x}} c_{x y}}}{c_{u}} .
$$

On the other hand, the watched chain $P^{\widetilde{V}}$ satisfies

$$
P^{\widetilde{V}}(u, v)=\frac{c_{u v}}{c_{u}}+\frac{c_{u x}}{c_{u}} \frac{c_{x v}}{\sum_{y \in V_{x}} c_{x y}} .
$$

Altogether, we see that $P^{\widetilde{G}}(u, v)=P^{\widetilde{V}}(u, v)$, completing the proof.

Well-separated sets. The following result is an important property of the resistance metric, crucial for our analysis.

Proposition 2.10. Consider a network $G(V)$ and its associated resistance metric $\left(V, R_{\mathrm{eff}}\right)$. Suppose that for some subset $S \subseteq V$, there is a partition $S=B_{1} \cup B_{2} \cup \cdots \cup B_{m}$ which satisfies the following properties.

1. For all $i=1,2, \ldots, m$ and for all $x, y \in B_{i}$, we have $R_{\mathrm{eff}}(x, y) \leqslant \varepsilon / 48$.

2. For all $i \neq j \in\{1,2, \ldots, m\}$, for all $x \in B_{i}$ and $y \in B_{j}$, we have $R_{\text {eff }}(x, y) \geqslant \varepsilon$. 
Then there is a subset $I \subseteq\{1,2, \ldots, m\}$ with $|I| \geqslant m / 2$ such that for all $i \in I$,

$$
R_{\mathrm{eff}}\left(B_{i}, S \backslash B_{i}\right) \geqslant \varepsilon / 24 \text {. }
$$

In order to prove Proposition 2.10, we need the following two ingredients.

Lemma 2.11. Suppose the network $H(W)$ can be partitioned into two disjoint parts $A$ and $B$ such that for some $\varepsilon>0$, and some vertices $u \in A$ and $v \in B$, we have

1. $R_{\mathrm{eff}}^{H}(u, v) \geqslant \varepsilon$, and

2. $R_{\mathrm{eff}}^{H}(u, x) \leqslant \varepsilon / 12$ for all $x \in A$, and $R_{\mathrm{eff}}^{H}(v, x) \leqslant \varepsilon / 12$ for all $x \in B$.

Then, $R_{\mathrm{eff}}^{H}(A, B) \geqslant \varepsilon / 6$.

Proof. Recall that by Thomson's Principle (see, e.g., [39, Ch. 2.4]), the effective resistance satisfies

$$
R_{\mathrm{eff}}(x, y)=\min _{f} \mathcal{E}(f), \text { where } \mathcal{E}(f)=\frac{1}{2} \sum_{x, y} f^{2}(x, y) r_{x y},
$$

and the minimum is over all unit flows from $x$ to $y$. Here, $r_{x y}=1 / c_{x y}$ is the edge resistance for $\{x, y\}$.

Suppose now that $R_{\text {eff }}^{H}(A, B)<\varepsilon / 6$. Then there exists a unit flow $f_{A B}$ from set $A$ to set $B$ such that $\mathcal{E}\left(f_{A B}\right)<\varepsilon / 6$. For $x \in A$, let $q_{x}$ be the amount of flow sent out from vertex $x$ in $f_{A B}$ and for $x \in B$, let $q_{x}$ be the amount of flow sent in to vertex $x$. Note that $\sum_{x \in A} q_{x}=\sum_{x \in B} q_{x}=1$.

Analogously, by assumption (2), there exist flows $\left\{f_{u x}: x \in A\right\}$ and $\left\{f_{x v}: x \in B\right\}$ such that $f_{x y}$ is a unit flow from $x$ to $y$ and $\mathcal{E}\left(f_{x y}\right) \leqslant \varepsilon / 12$. We next build a flow $f$ such that

$$
f=f_{A B}+\sum_{w \in A} q_{w} f_{u w}+\sum_{z \in B} q_{z} f_{z v} .
$$

We see that $f$ is indeed a unit flow from $u$ to $v$. Furthermore, by Cauchy-Schwartz,

$$
\begin{aligned}
\mathcal{E}(f) & =\frac{1}{2} \sum_{x, y} f^{2}(x, y) r_{x y} \\
& =\frac{1}{2} \sum_{x, y} r_{x y}\left(f_{A B}(x, y)+\sum_{w \in A} q_{w} f_{u w}(x, y)+\sum_{z \in B} q_{z} f_{z v}(x, y)\right)^{2} \\
& \leqslant \frac{3}{2} \sum_{x, y} r_{x y}\left(f_{A B}^{2}(x, y)+\sum_{w \in A} q_{w} f_{u w}^{2}(x, y)+\sum_{z \in B} q_{z} f_{z v}^{2}(x, y)\right) \\
& =3\left(\mathcal{E}\left(f_{A B}\right)+\sum_{w \in A} q_{w} \mathcal{E}\left(f_{u w}\right)+\sum_{z \in B} q_{z} \mathcal{E}\left(f_{z v}\right)\right) \\
& <\varepsilon
\end{aligned}
$$

This contradicts assumption (1), completing the proof. 
Lemma 2.12. For any network $G(V)$, the following holds. If there is a subset $S \subseteq V$ and a value $\varepsilon>0$ such that $R_{\mathrm{eff}}(u, v) \geqslant \varepsilon$ for all $u, v \in S$, then there is a subset $S^{\prime} \subseteq S$ with $\left|S^{\prime}\right| \geqslant|S| / 2$ such that for every $v \in S^{\prime}$,

$$
R_{\mathrm{eff}}(v, S \backslash\{v\}) \geqslant \varepsilon / 4 \text {. }
$$

Proof. Consider the reduced network $\widetilde{G}$ on the vertex set $S$, as defined in Lemma 2.9, Let the new conductances be denoted $\tilde{c}_{x y}$ for $x, y \in S$. By Lemma 2.9, our initial assumption that $R_{\text {eff }}(u, v) \geqslant \varepsilon$ for all $u, v \in S$ implies that $R_{\text {eff }}^{\widetilde{G}}(u, v) \geqslant \varepsilon$ for all $u, v \in S$.

Let $n=|S|$. Foster's Theorem [26] (see also [53]) states that

$$
\frac{1}{2} \sum_{u \neq v \in S} R_{\mathrm{eff}}^{\widetilde{G}}(u, v) \tilde{c}_{u, v}=n-1 .
$$

Combined with the fact that $R_{\text {eff }}^{\widetilde{G}}(u, v) \geqslant \varepsilon$, this yields

$$
\frac{1}{2} \sum_{u \neq v \in S} \tilde{c}_{u v} \leqslant \frac{n}{\varepsilon} .
$$

In particular, there exists a subset $S^{\prime} \subseteq S$ with $\left|S^{\prime}\right| \geqslant n / 2$ such that for all $v \in S^{\prime}$,

$$
\sum_{u \in S \backslash\{v\}} \tilde{c}_{u v} \leqslant \frac{4}{\varepsilon} .
$$

It follows that for every $v \in S^{\prime}$, we have $C_{\text {eff }}^{\widetilde{G}}(v, S \backslash\{v\}) \leqslant 4 / \varepsilon$, hence

$$
R_{\mathrm{eff}}(v, S \backslash\{v\})=R_{\mathrm{eff}}^{\widetilde{G}}(v, S \backslash\{v\}) \geqslant \varepsilon / 4 \text {. }
$$

Proof of Proposition [2.10. For each $i \in\{1,2, \ldots, m\}$, choose some $v_{i} \in B_{i}$. By assumption (2), $R_{\text {eff }}\left(v_{i}, v_{j}\right) \geqslant \varepsilon$ for $i \neq j$. Thus applying Lemma 2.12 , we find a subset $I \subseteq\{1,2, \ldots, m\}$ with $|I| \geqslant m / 2$ and such that for every $i \in I$, we have

$$
R_{\text {eff }}\left(v_{i},\left\{v_{1}, \ldots, v_{m}\right\} \backslash\left\{v_{i}\right\}\right) \geqslant \varepsilon / 4 .
$$

We claim that this subset $I$ satisfies the conclusion of the proposition.

To this end, fix $i \in I$, and let $\tilde{G}$ be the quotient network formed by gluing $\left\{v_{1}, \ldots, v_{m}\right\} \backslash\left\{v_{i}\right\}$ into a single vertex $\tilde{v}$. By (34), we have $R_{\text {eff }}^{\tilde{G}}\left(v_{i}, \tilde{v}\right) \geqslant \varepsilon / 4$. Now let,

$$
\tilde{B}=\left(\{\tilde{v}\} \cup \bigcup_{j \neq i} B_{j}\right) \backslash\left\{v_{i}\right\}_{i \in I} .
$$

Consider any $x \in \tilde{B}$ with $x \neq \tilde{v}$. Then $x \in B_{j}$ for some $j \neq i$, hence by assumption (1), we conclude that,

$$
R_{\text {eff }}^{\tilde{G}}(x, \tilde{v}) \leqslant R_{\text {eff }}\left(x, v_{j}\right) \leqslant \varepsilon / 48 .
$$


We may now apply Lemma 2.11 to the sets $B_{i}$ and $\tilde{B}$ in $\tilde{G}$ (with respective vertices $v_{i}$ and $\tilde{v}$ ) to conclude that

$$
R_{\mathrm{eff}}^{\tilde{G}}\left(B_{i}, \tilde{B}\right) \geqslant \varepsilon / 24 \text {. }
$$

But the preceding line immediately yields,

$$
R_{\mathrm{eff}}\left(B_{i}, S \backslash B_{i}\right) \geqslant \varepsilon / 24,
$$

finishing the proof.

We end this section with the following simple lemma.

Lemma 2.13. For any network $G(V)$, if $A, B_{1}, B_{2} \subseteq V$ are disjoint, then

$$
R_{\mathrm{eff}}\left(A, B_{1} \cup B_{2}\right) \geqslant \frac{R_{\mathrm{eff}}\left(A, B_{1}\right) \cdot R_{\mathrm{eff}}\left(A, B_{2}\right)}{R_{\mathrm{eff}}\left(A, B_{1}\right)+R_{\mathrm{eff}}\left(A, B_{2}\right)} .
$$

Proof. By considering the quotient graph, the lemma can be reduced to the case when $A=\{u\}$. Let $\left\{S_{t}\right\}$ be the discrete-time random walk on the network and define

$$
T_{B}=\min \left\{t \geqslant 0: S_{t} \in B\right\} \text { and } T_{B}^{+}=\min \left\{t \geqslant 1: S_{t} \in B\right\} \text { for } B \subseteq V .
$$

It is clear that for a random walk started at $u$, we have

$$
\mathbb{P}_{u}\left(T_{u}^{+}>T_{B_{1} \cup B_{2}}\right) \leqslant \mathbb{P}_{u}\left(T_{u}^{+}>T_{B_{1}}\right)+\mathbb{P}_{u}\left(T_{u}^{+}>T_{B_{2}}\right) .
$$

Combined with (33), this gives

$$
\frac{1}{R_{\text {eff }}\left(u, B_{1} \cup B_{2}\right)} \leqslant \frac{1}{R_{\text {eff }}\left(u, B_{1}\right)}+\frac{1}{R_{\text {eff }}\left(u, B_{2}\right)},
$$

yielding the desired inequality.

\subsection{The Gaussian free field}

We recall the graph Laplacian $\Delta: \ell^{2}(V) \rightarrow \ell^{2}(V)$ defined by

$$
\Delta f(x)=c_{x} f(x)-\sum_{y} c_{x y} f(y)
$$

Consider a connected network $G(V)$. Fix a vertex $v_{0} \in V$, and consider the random process $\mathcal{X}=\left\{\eta_{v}\right\}_{v \in V}$, where $\eta_{v_{0}}=0$, and $\mathcal{X}$ has density proportional to

$$
\exp \left(-\frac{1}{2}\langle\mathcal{X}, \Delta \mathcal{X}\rangle\right)=\exp \left(-\frac{1}{4} \sum_{u, v} c_{u v}\left|\eta_{u}-\eta_{v}\right|^{2}\right) .
$$

The process $\mathcal{X}$ is called the Gaussian free field (GFF) associated with $G$. The next lemma is known, see, e.g., Theorem 9.20 of [28]. We include the proof for completeness.

Lemma 2.14. For any connected network $G(V)$, if $\mathcal{X}=\left\{\eta_{v}\right\}_{v \in V}$ is the associated GFF, then for all $u, v \in V$,

$$
\mathbb{E}\left(\eta_{u}-\eta_{v}\right)^{2}=R_{\mathrm{eff}}(u, v) .
$$


Proof. From (35), and the fact that the Laplacian is positive semi-definite, it is clear that $\mathcal{X}$ is a Gaussian process. Let $\Gamma_{v_{0}}(u, v)=\mathbb{E}_{u} L_{T_{0}}^{v}$, where $T_{0}$ is the hitting time for $v_{0}$ as in Theorem 1.14, From Lemma 2.1, we have

$$
\Gamma_{v_{0}}(u, v)=\frac{1}{2}\left(R_{\mathrm{eff}}\left(v_{0}, u\right)+R_{\mathrm{eff}}\left(v_{0}, v\right)-R_{\mathrm{eff}}(u, v)\right)
$$

Let $\widetilde{\Delta}$ and $\widetilde{\Gamma}_{v_{0}}$, respectively, be the matrices $\Delta$ and $\Gamma_{v_{0}}$ with the row and column corresponding to $v_{0}$ removed. Appealing to (35), if we can show that $\widetilde{\Delta} \widetilde{\Gamma}_{v_{0}}=I$, it follows that $\Gamma_{v_{0}}$ is the covariance matrix for $\mathcal{X}$. In this case, comparing (37) to

$$
\mathbb{E}\left(\eta_{u} \eta_{v}\right)=\frac{1}{2}\left(\mathbb{E} \eta_{u}^{2}+\mathbb{E} \eta_{v}^{2}-\mathbb{E}\left(\eta_{u}-\eta_{v}\right)^{2}\right)
$$

and using $\eta_{v_{0}}=0$, we see that (36) follows.

In order to demonstrate $\widetilde{\Delta} \widetilde{\Gamma}_{v_{0}}=I$, we consider $u, v$ such that $v_{0} \notin\{u, v\}$. Conditioning on the first step of the walk from $u$ gives,

$$
\begin{aligned}
c_{u} \Gamma_{v_{0}}(u, v)=c_{u} \mathbb{E}_{u} L_{T_{0}}^{v} & =\mathbf{1}_{\{u=v\}}+\sum_{w} c_{u w} \mathbb{E}_{w} L_{T_{0}}^{v} \\
& =\mathbf{1}_{\{u=v\}}+\sum_{w} c_{u w} \Gamma_{v_{0}}(v, w)
\end{aligned}
$$

On the other hand, by definition of the Laplacian,

$$
\left(\Delta \Gamma_{v_{0}}\right)(u, v)=c_{u} \Gamma_{v_{0}}(u, v)-\sum_{w} c_{u w} \Gamma_{v_{0}}(v, w)=\mathbf{1}_{\{u=v\}},
$$

where the latter equality is precisely (38). Thus $\widetilde{\Delta} \widetilde{\Gamma}_{v_{0}}=I$, completing the proof.

A geometric identity. In what follows, for a set of points $Y$ lying in some Hilbert space, we use $\operatorname{aff}(Y)$ to denote their affine hull, i.e. the closure of $\left\{\sum_{i=1}^{n} \alpha_{i} y_{i}: n \geqslant 1, y_{i} \in Y, \sum_{i=1}^{n} \alpha_{i}=1\right\}$. Of course, when $Y$ contains the origin, $\operatorname{aff}(Y)$ is simply the linear span of $Y$.

Lemma 2.15. For any network $G(V)$, if $\mathcal{X}=\left\{\eta_{v}\right\}_{v \in V}$ is the $G F F$ associated with $G$, then for any $w \in V$ and subset $S \subseteq V$,

$$
\sqrt{R_{\text {eff }}(w, S)}=\operatorname{dist}_{L^{2}}\left(\eta_{w}, \operatorname{aff}\left(\left\{\eta_{u}\right\}_{u \in S}\right)\right) .
$$

Proof. Since the statement of the lemma is invariant under translation, we may assume that the GFF is defined with respect to some $v_{0} \in S$.

In this case, by the definition in (35), the GFF for $G / S$ has density proportional to

$$
\exp \left(-\frac{1}{4}\left(\sum_{u, v \notin S} c_{u v}\left|\eta_{u}-\eta_{v}\right|^{2}+\sum_{u \notin S} c_{v_{S} u}\left|\eta_{u}\right|^{2}\right)\right),
$$

i.e. the GFF on $G / S$ is precisely the initial Gaussian process $\mathcal{X}$ conditioned on the linear subspace $A_{S}=\left\{\eta_{v}=\eta_{v_{0}}=0: v \in S\right\}$. 
Using (31) and Lemma 2.14, we have

$$
R_{\mathrm{eff}}(w, S)=R_{\mathrm{eff}}^{G / S}\left(w, v_{S}\right)=\mathbb{E}\left[\left|\eta_{w}-\eta_{v_{0}}\right|^{2} \mid A_{S}\right]=\mathbb{E}\left[\left|\eta_{w}\right|^{2} \mid A_{S}\right] .
$$

To compute the latter expectation, write $\eta_{w}=Y+Y^{\prime}$, where $Y^{\prime} \in \operatorname{span}\left(\left\{\eta_{v}\right\}_{v \in S}\right)$ and $\mathbb{E}\left(Y Y^{\prime}\right)=0$. It follows immediately that

$$
\operatorname{dist}_{L^{2}}\left(\eta_{w}, \operatorname{aff}\left(\left\{\eta_{u}\right\}_{u \in S}\right)\right)=\sqrt{\mathbb{E}\left[Y^{2}\right]}=\sqrt{\mathbb{E}\left[\left|\eta_{w}\right|^{2} \mid A_{S}\right]}
$$

completing the proof.

\section{$3 \quad$ Majorizing measures}

We now review the relevant parts of the majorizing measure theory. One is encouraged to consult the book [52] for further information. In Section 1, we saw Talagrand's $\gamma_{2}$ functional. For our purposes, it will be more convenient to work with a different value that is equivalent to the functional $\gamma_{2}$, up to universal constants. In Section 3.2, we discuss separated trees, and prove a number of standard properties about such objects. In Section 3.3, we present a deterministic algorithm for computing $\gamma_{2}(X, d)$ for any finite metric space $(X, d)$. Finally, in Section 3.4, we specialize the theory of Gaussian processes and trees to the case of GFFs. There, we will use the geometric properties proved in Sections 2.3 and 2.4 .

Before we begin, we attempt to give some rough intuition about the role of trees in the majorizing measures theory. A good reference for this material is [27]. A tree of subsets of $X$ is a finite collection $\mathcal{F}$ of subsets with the property that for all $A, B \in \mathcal{F}$, either $A \cap B=\emptyset$, or $A \subseteq B$, or $B \subseteq A$. A set $B$ is a child of $A$ if $B \subseteq A, B \neq A$, and

$$
C \in \mathcal{F}, B \subseteq C \subseteq A \Longrightarrow C=B \text { or } C=A .
$$

We assume that $X \in \mathcal{F}$, and $X$ is referred to as the root of the tree $\mathcal{F}$. To each $A \in \mathcal{F}$, we use $N(A)$ to denote the number of children of $A$. A branch of $\mathcal{F}$ is a sequence $A_{1} \supset A_{2} \supset \cdots$ such that each $A_{k+1}$ is a child of $A_{k}$. A branch is maximal if it is not contained in a longer branch. We will assume additionally that every maximal branch terminates in a singleton set $\{x\}$ for $x \in X$.

Let $\left\{\eta_{x}\right\}_{x \in X}$ be a centered Gaussian process with $X$ finite, and let $d(x, y)=\sqrt{\mathbb{E}\left(\eta_{x}-\eta_{y}\right)^{2}}$. The basic premise of the tree interpretation of the majorizing measures theory is that one can assign a measure of "size" to any tree of subsets in $X$, and this size provides a lower bound on $\mathbb{E} \sup _{x \in X} \eta_{x}$. The majorizing measures theorem then claims that the value of the optimal such tree is within absolute constants of the expected supremum. The size of the tree (see (39) ) can be defined using only the metric structure of $(X, d)$, without reference to the underlying Gaussian process. Thus much of the theorems in this section are stated for general metric spaces.

The tree of subsets is meant to capture the structure of $(X, d)$ at all scales simultaneously. In general, to obtain a multi-scale lower bound on the expected supremum of the process, one arranges so that the diameter of the subsets decreases exponentially as one goes down the tree, and all subsets at one level of the tree are separated by a constant fraction of their diameter (see Definitions 3.1 and 3.8 below). This allows a certain level of independence between different branches of the tree which is exploited in the lower bounds. Much of this section is devoted to proving that one can construct a near-optimal tree with a number of regularity properties that will be crucial to our approach in Section 4 , 


\subsection{Trees, measures, and functionals}

Let $(X, d)$ be an arbitrary metric space.

Definition 3.1. For values $q \in \mathbb{N}$ and $\alpha, \beta>0$, and $r \geqslant 2$, a tree of subsets $\mathcal{F}$ in $X$ is called a $(q, r, \alpha, \beta)$-tree if to each $A \in \mathcal{F}$, one can associate a number $n(A) \in \mathbb{Z}$ such that the following three conditions are satisfied.

1. For all children $B$ of $A$, we have $n(B) \leqslant n(A)-q$.

2. If $B$ and $B^{\prime}$ are two distinct children of $A$, then $d\left(B, B^{\prime}\right) \geqslant \beta r^{n(A)-1}$.

3. $\operatorname{diam}(A) \leqslant \alpha r^{n(A)}$.

We will refer to a $\left(q, r, 4, \frac{1}{2}\right)$-tree as simply a $(q, r)$-tree.

The $r$-size of a tree of subsets $\mathcal{F}$, written $\operatorname{size}_{r}(\mathcal{F})$, is defined as the infimum of

$$
\sum_{k \geqslant 1} r^{n\left(A_{k}\right)} \sqrt{\log ^{+} N\left(A_{k}\right)}
$$

over all possible maximal branches of $\mathcal{F}$, where we use the notation $\log ^{+} x=\log x$ for $x \neq 0$, and $\log ^{+}(0)=0$.

To connect trees of subsets with the $\gamma_{2}$ functional, we recall the relationship with majorizing measures. The next result is from [51, Thm. 1.1]

Theorem 3.2. For every metric space $(X, d)$, we have

$$
\gamma_{2}(X, d) \asymp \inf \sup _{x \in X} \int_{0}^{\infty}\left(\log \frac{1}{\mu(B(x, \varepsilon))}\right)^{1 / 2} d \varepsilon,
$$

where $B(x, \varepsilon)$ is the closed ball of radius $\varepsilon$ about $x$, and the infimum is over all finitely supported probability measures on $X$.

We will also need the following theorem due to Talagrand (see Proposition 4.3 of [50] and also Theorem T5 of [27].) We will employ it now and also in Section 3.3.

Theorem 3.3. There is a value $r_{0} \geqslant 2$ such that the following holds. Let $(X, d)$ be a finite metric space, and $r \geqslant r_{0}$. Assume there is a family of functions $\left\{\varphi_{i}: X \rightarrow \mathbb{R}_{+}: i \in \mathbb{Z}\right\}$ such that the following conditions hold for some $\beta>0$.

1. $\varphi_{i}(x) \geqslant \varphi_{i-1}(x)$ for all $i \in \mathbb{Z}$ and $x \in X$.

2. If $t_{1}, t_{2}, \ldots, t_{N} \in B\left(s, r^{j}\right)$ are such that $d\left(t_{i}, t_{i^{\prime}}\right) \geqslant r^{j-1}$ for $i \neq i^{\prime}$, then

$$
\varphi_{j}(s) \geqslant \beta r^{j} \sqrt{\log N}+\min \left\{\varphi_{j-2}\left(t_{i}\right): i=1,2, \ldots, N\right\} .
$$

Under these conditions,

$$
\gamma_{2}(X, d) \lesssim r, \beta \sup _{x \in X, i \in \mathbb{Z}} \varphi_{i}(x)
$$


The preceding two theorems allow us to present the following connection between trees and $\gamma_{2}$. Such a connection is well-known (see, e.g. [49]), but we record the proofs here for completeness, and for the precise quantitative bounds we will use in future sections.

Lemma 3.4. There is a value $r_{0} \geqslant 2$ such that for every finite metric space $(X, d)$, and every $r \geqslant r_{0}$, we have

$$
\gamma_{2}(X, d) \lesssim_{r} \sup \left\{\operatorname{size}_{r}(\mathcal{F}): \mathcal{F} \text { is a }\left(1, r, 4, \frac{1}{2}\right) \text {-tree in } X\right\} .
$$

Proof. First, for a subset $S \subseteq X$, let

$$
\theta(S)=\sup \left\{\operatorname{size}_{r}(\mathcal{F}): \mathcal{F} \text { is a }\left(1, r, 4, \frac{1}{2}\right) \text {-tree in } X\right\} .
$$

Then define, for every $i \in \mathbb{Z}$ and $x \in X$, define

$$
\varphi_{i}(x)=\theta\left(B\left(x, 2 r^{i}\right)\right) .
$$

where $B(x, R)$ is the closed ball of radius $R$ about $x \in X$. We now wish to verify that the conditions of Theorem 3.3 hold for $\left\{\varphi_{i}\right\}$. Condition (1) is immediate.

Assume that $r \geqslant 8$. Given $t_{1}, t_{2}, \ldots, t_{N}$ as in condition (2) of Theorem 3.3. consider the set $A=B\left(s, 2 r^{j}\right)$ which has diameter bounded by $4 r^{j}$, and the disjoint subset sets of $A$ given by $A_{i}=B\left(t_{i}, 2 r^{j-2}\right)$ which each have diameter bounded by $4 r^{j-2}$, and which satisfy $d\left(A_{i}, A_{j}\right) \geqslant r^{j-1} / 2$ for $i \neq j$. We also have $A_{i} \subseteq A$ for each $i \in\{1, \ldots, N\}$.

Taking the tree of subsets with $\operatorname{root} A, n(A)=j$, and children $\left\{A_{i}\right\}_{i=1}^{N}$, and in each $A_{i}$ a tree which achieves value at least $\theta\left(A_{i}\right)=\theta\left(B\left(t_{i}, 2 r^{j-2}\right)\right)=\varphi_{j-2}(i)$, we see immediately that

$$
\varphi_{j}(s)=\theta\left(B\left(s, 2 r^{j}\right)\right) \geqslant r^{j} \sqrt{\log N}+\min \left\{\varphi_{j-2}\left(t_{i}\right): i=1,2, \ldots, N\right\},
$$

confirming condition (2) of Theorem 3.3. Applying the theorem, it follows that $\gamma_{2}(X, d) \lesssim_{r} \theta(X)$, proving (40).

We will need the upper bound (40) to hold for $\left(2, r, 4, \frac{1}{2}\right)$-trees. Toward this end, we state a version of [49, Thm 3.1]. The theorem there is only proved for $\alpha=1$ and $\beta=\frac{1}{2}$, but it is straightforward to see that it works for all values $\alpha, \beta>0$ since the proof merely proceeds by choosing an appropriate subtree of the given tree; the values $\alpha$ and $\beta$ are not used.

Theorem 3.5. For every metric space $(X, d)$, the following holds. For every $\alpha, \beta, r>0$ and $q \in \mathbb{N}$, and for every $(1, r, \alpha, \beta)$-tree $\mathcal{F}$ in $X$, there exists a $(q, r, \alpha, \beta)$-tree $\mathcal{F}^{\prime}$ in $X$ such that

$$
\operatorname{size}_{r}(\mathcal{F}) \lesssim q \cdot \operatorname{size}_{r}\left(\mathcal{F}^{\prime}\right) .
$$

Combining Theorem 3.5 with Lemma 3.4 yields the following upper bound using $(2, r)$-trees.

Corollary 3.6. There is a value $r_{0} \geqslant 2$ such that for every finite metric space $(X, d)$, and every $r \geqslant r_{0}$, we have

$$
\gamma_{2}(X, d) \lesssim_{r} \sup \left\{\operatorname{size}_{r}(\mathcal{F}): \mathcal{F} \text { is a }\left(2, r, 4, \frac{1}{2}\right) \text {-tree in } X\right\} \text {. }
$$

Now we move onto a lower bound on $\gamma_{2}$.

Lemma 3.7. There is a value $r_{0} \geqslant 2$ such that for every finite metric space $(X, d)$, and every $r \geqslant r_{0}$, we have

$$
\gamma_{2}(X, d) \gtrsim \sup \left\{\operatorname{size}_{r}(\mathcal{F}): \mathcal{F} \text { is a }\left(1, r, 8, \frac{1}{6}\right) \text {-tree }\right\}
$$


Proof. We will show for any probability measure $\mu$ on $X$ and any $\left(1, r, 8, \frac{1}{6}\right)$-tree $\mathcal{F}$ in $X$, we have

$$
\operatorname{size}_{r}(\mathcal{F}){ }_{r} \sup _{x \in X} \int_{0}^{\infty}\left(\log \frac{1}{\mu(B(x, \varepsilon))}\right)^{1 / 2} d \varepsilon
$$

The basic idea is that if $A_{1}, A_{2}, \ldots A_{k}$ are children of $A$, in $\mathcal{F}$, then the sets $B\left(A_{i}, \frac{1}{20} r^{n(A)-1}\right)$ are disjoint by property (2) of Definition 3.1, where we write $B(S, R)=\{x \in X: d(x, S) \leqslant R\}$. Thus one of these sets $A_{i}$ has $\mu\left(B\left(A_{i}, \frac{1}{20} r^{n(A)-1}\right)\right) \leqslant 1 / N(A)$.

Thus we may find a finite sequence of sets, starting with $A^{(0)}=X$ such that $A^{(i+1)}$ is a child $A^{(i)}$ and

$$
\mu\left(B\left(A^{(i+1)}, \frac{1}{20} r^{n\left(A^{(i)}\right)-1}\right)\right) \leqslant 1 / N\left(A^{(i)}\right) .
$$

Since every maximal branch in a tree of subsets terminates in a singleton, the sequence ends with some set $A^{\prime}=A^{(h)}=\{x\}$. By construction, we have

$$
\mu\left(B\left(x, \frac{1}{20} r^{n\left(A^{\prime}\right)-1}\right)\right) \leqslant \frac{1}{N\left(A^{\prime}\right)} .
$$

Thus, assuming $r \geqslant 40$,

$$
r^{n\left(A^{\prime}\right)-2} \sqrt{\log ^{+} N\left(A^{\prime}\right)} \leqslant \int_{r^{n\left(A^{\prime}\right)-2}}^{\frac{1}{20} r^{n\left(A^{\prime}\right)-1}} \sqrt{\frac{1}{\log \mu(B(x, \varepsilon))}} d \varepsilon .
$$

By property of Definition 3.1, the intervals $\left(r^{n(A)-2}, \frac{1}{20} r^{n(A)-1}\right)$ are disjoint for different sets $A \in \mathcal{F}$ with $x \in A$, thus summing (42) yields

$$
\operatorname{size}_{r}(\mathcal{F}) \lesssim r \sum_{A \in \mathcal{F}: x \in A} r^{n(A)-2} \sqrt{\log ^{+} N(A)} \leqslant \int_{0}^{\infty} \sqrt{\frac{1}{\log \mu(B(x, \varepsilon))}} d \varepsilon,
$$

completing the proof.

\subsection{Separated trees}

Let $(X, d)$ be an arbitrary metric space. Consider a finite, connected, graph-theoretic tree $\mathcal{T}=$ $(V, E)$ (i.e., a connected, acyclic graph) such that $V \subseteq X$, with a fixed root $z \in V$, and a mapping $s: V \rightarrow \mathbb{Z}$. Abusing notation, we will sometimes use $\mathcal{T}$ for the vertex set of $\mathcal{T}$. For a vertex $x \in \mathcal{T}$, we use $\mathcal{T}_{x}$ to denote the subtree rooted at $x$, and we use $\Gamma(x)$ to denote the set of children 11 of $x$ with respect to the root $z$. Finally, we write $\Delta(x)=|\Gamma(x)|+1$ for all $x \in \mathcal{T}$.

Let $\mathcal{L}$ be the set of leaves of $\mathcal{T}$. For any $v \in \mathcal{T}$, let $\mathcal{P}(v)=\{z, \ldots, v\}$ denote the set of nodes on the unique path from the root to $v$. For a pair of nodes $u, v \in \mathcal{T}$, we use $\mathcal{P}(u, v)$ to denote the sequence of nodes on the unique path from $u$ to $v$. If $u$ is the parent of $v$, we write $u=p(v)$ and in particular we write $z=p(z)$. For any such pair $(\mathcal{T}, s)$ and $r \geqslant 2$, we define the value of $(\mathcal{T}, s)$ by

$$
\operatorname{val}_{r}(\mathcal{T}, s)=\inf _{\ell \in \mathcal{L}} \sum_{v \in \mathcal{P}(\ell)} r^{s(v)} \sqrt{\log \Delta(v)} .
$$

The following definition will be central.

\footnotetext{
${ }^{1}$ Formally, these are precisely the neighbors of $x$ in $\mathcal{T}$ whose unique path to the root $z$ passes through $x$.
} 
Definition 3.8. For a value $r \geqslant 2$, we say that the pair $(\mathcal{T}, s)$ is an $r$-separated tree in $(X, d)$ if it satisfies the following conditions for all $x \in \mathcal{T}$.

1. For all $y \in \Gamma(x), s(y) \leqslant s(x)-2$.

2. For all $u, v \in \Gamma(x)$, we have $d\left(x, \mathcal{T}_{u}\right) \geqslant \frac{1}{2} r^{s(x)-1}$ and $d\left(\mathcal{T}_{u}, \mathcal{T}_{v}\right) \geqslant \frac{1}{2} r^{s(x)-1}$.

3. $\operatorname{diam}\left(\mathcal{T}_{x}\right) \leqslant 4 r^{s(x)}$.

We remark that our separated tree is a slightly different version of the $(2, r)$-tree introduced in the preceding section. The main difference is that the nodes of our separated tree are point in the metric space $X$, whereas a node in a $(2, r)$-tree is a subset of $X$. Our definition is tailored for the application in Section 4 .

Not surprisingly, we have a similar version of the above theorem for separated trees.

Theorem 3.9. For some $r_{0} \geqslant 2$ and every $r \geqslant r_{0}$, and any metric space $(X, d)$, we have

$$
\sup _{\mathcal{T}} \operatorname{val}_{r}(\mathcal{T}, s) \asymp_{r} \gamma_{2}(X, d),
$$

where the supremum is over all $r$-separated trees in $X$.

Theorem 3.9 follows from Corollary 3.6 and the following lemma.

Lemma 3.10. Consider $r \geqslant 8$ and any metric space $(X, d)$. For any $(2, r)$-tree $\mathcal{F}$, there is an $r$ separated tree $\mathcal{T}$ such that $\operatorname{size}_{r}(\mathcal{F})=\operatorname{val}_{r}(\mathcal{T})$. Also, for any $r$-separated tree $\mathcal{T}$, there is a $(2, r)$-tree $\mathcal{F}$ such that $\operatorname{size}_{r}(\mathcal{F}) \geqslant \operatorname{val}_{r}(\mathcal{T})-r \operatorname{diam}(X)$.

Proof. We only prove the first half of the statement, since the second half can be obtained by reversing the construction. The additive factor $-r \operatorname{diam}(X)$ is due to the slight difference in the definitions of the value for a separated tree and the size for a $(2, r)$-tree (see (43) and (39)).

Let $\mathcal{F}$ be a $(2, r)$-tree on $(X, d)$. For each $A \in \mathcal{F}$ with $N(A) \geqslant 1$, we select one child $c(A)$ and an arbitrary point $v_{A} \in c(A)$. We now construct the separated tree $\mathcal{T}$. Its vertex set is a subset of $\left\{v_{A}: A \in \mathcal{F}\right\}$. The root of $\mathcal{T}$ is $v_{X}$, and its children are $\left\{v_{B}: B\right.$ is a child of $X$ with $\left.B \neq c(X)\right\}$. In general, if $v_{A}$ is a node of $\mathcal{T}$, then its children are the points $\left\{v_{B}: B\right.$ is a child of $A$ with $\left.B \neq c(A)\right\}$. Finally, for $v_{A} \in \mathcal{T}$, we put $s\left(v_{A}\right)=n(A)$.

Let us first verify that $\mathcal{T}$ is an $r$-separated tree. Condition (1) of Definition 3.8 holds because if $y$ is a child of $v_{A} \in \mathcal{T}$, then $y=v_{B}$ for some child $B$ of $A$ (in $\mathcal{F}$ ), which implies $s(y)=n(B) \leqslant$ $n(A)-2=s\left(v_{A}\right)-2$. Secondly, If $v_{A}$ is a node with children $v_{B_{1}}, v_{B_{2}}, \ldots, v_{B_{k}}$, then clearly by Definition 3.1,

$$
\begin{aligned}
& d\left(v_{A}, \mathcal{T}_{v_{B_{i}}}\right) \geqslant d\left(c(A), B_{i}\right) \geqslant \frac{1}{2} r^{s\left(v_{A}\right)-1}, \\
& d\left(\mathcal{T}_{v_{B_{i}}}, \mathcal{T}_{v_{B_{j}}}\right) \geqslant d\left(B_{i}, B_{j}\right) \geqslant \frac{1}{2} r^{s\left(v_{A}\right)-1}
\end{aligned}
$$

verifying condition (2) of Definition 3.8 ,

Thirdly, if $x_{A} \in \mathcal{T}$, then for any child $x_{B}$ of $x_{A}$, we know $B$ is a child of $A$, hence

$$
\operatorname{diam}\left(\mathcal{T}_{x_{B}}\right) \leqslant \operatorname{diam}(B) \leqslant 4 r^{n(A)}=4 r^{s\left(x_{A}\right)},
$$

using property (3) of a $q$-tree. This verifies condition (3) of Definition 3.8.

Finally, observe that for every non-leaf node $v_{A} \in \mathcal{T}$, we have $\Delta\left(v_{A}\right)=\left|\Gamma\left(v_{A}\right)\right|+1=N(A)$, and for leaves, we have $\log \Delta\left(v_{A}\right)=\log ^{+} N(A)=0$. It follows that $\operatorname{val}_{r}(\mathcal{T}, s)=\operatorname{size}_{r}(\mathcal{F})$, completing the proof. 


\subsubsection{Additional structure}

We now observe that we can take our separated trees to have some additional properties. Say that an $r$-separated tree $(\mathcal{T}, s)$ is $C$-regular for some $C \geqslant 1$, if it satisfies, for every $v \in \mathcal{T} \backslash \mathcal{L}$,

$$
\Delta(v) \geqslant \exp \left(C^{2} r^{2} 4^{s(z)-s(v)}\right)
$$

Lemma 3.11. For every $C \geqslant 1$ and $r \geqslant 4$, for every $r$-separated tree $(\mathcal{T}, s)$ in $X$, if

$$
\operatorname{val}_{r}(\mathcal{T}, s) \geqslant 4 C r^{s(z)+1}
$$

then there is a $C$-regular $r$-separated tree $\left(\mathcal{T}^{\prime}, s^{\prime}\right)$ in $X$ with

$$
\frac{1}{2} \operatorname{val}_{r}(\mathcal{T}, s) \leqslant \operatorname{val}_{r}\left(\mathcal{T}^{\prime}, s^{\prime}\right) \leqslant \operatorname{val}_{r}(\mathcal{T}, s)
$$

Proof. Consider the following operation on an $r$-separated tree $(\mathcal{T}, s)$. For $x \in \mathcal{T} \backslash \mathcal{L}$, consider a new $r$-separated tree $\left(\mathcal{T}^{\prime}, s^{\prime}\right)=\Phi_{x}(\mathcal{T}, s)$, which is defined as follows. Let $u$ be the child of $x$ and let $S$ contain the remaining children such that

$$
\operatorname{val}_{r}\left(\mathcal{T}_{u},\left.s\right|_{\mathcal{T}_{u}}\right) \leqslant \operatorname{val}_{r}\left(\mathcal{T}_{v},\left.s\right|_{\mathcal{T}_{v}}\right) \text { for all } v \in S
$$

where $\mathcal{T}_{u}$ is the subtree of $\mathcal{T}$ rooted at $u$ and containing all its descendants, and $\left.s\right|_{\mathcal{T}_{u}}$ is the restriction of $s$ on the subtree $\mathcal{T}_{u}$. Consider the tree $\mathcal{T}^{\prime}$ that results from deleting all the nodes in $S$, as well as the subtrees under them, and then contracting the edge $(x, u)$. We also put $s^{\prime}(x)=s(u)$ and $s^{\prime}(y)=s(y)$ for all $y \in \mathcal{T}^{\prime}$.

As long as there is a node $x \in \mathcal{T} \backslash \mathcal{L}$ which violates (44) (for the current $\left(\mathcal{T}^{\prime}, s^{\prime}\right)$ ), we iterate this procedure (namely, we replace $\left(\mathcal{T}^{\prime}, s^{\prime}\right)$ by $\Phi_{x}\left(\mathcal{T}^{\prime}, s^{\prime}\right)$ ). It is clear that we end with a $C$-regular tree $\left(\mathcal{T}^{\prime}, s^{\prime}\right)$. Note that different choices of $x$ at each stage will lead to different outcomes, but the following proof shows that all of them satisfy the required condition.

It is also straightforward to verify that for any $\ell \in \mathcal{L}^{\prime}$, we have

$$
\begin{aligned}
\sum_{v \in \mathcal{P}_{\mathcal{T}^{\prime}}(\ell)} r^{s^{\prime}(v)} \sqrt{\log \Delta_{\mathcal{T}^{\prime}}(v)} & \geqslant \sum_{v \in \mathcal{P}_{\mathcal{T}}(\ell)} r^{s(v)} \sqrt{\log \Delta_{\mathcal{T}}(v)}-C r \sum_{v \in P_{\mathcal{T}}(\ell)} r^{s(v)} 2^{s(z)-s(v)} \\
& \geqslant \sum_{v \in \mathcal{P}_{\mathcal{T}}(\ell)} r^{s(v)} \sqrt{\log \Delta_{\mathcal{T}}(v)}-C r^{s(z)+1} \sum_{k=0}^{\infty} 2^{2 k} r^{-2 k} \\
& \geqslant \sum_{v \in \mathcal{P}_{\mathcal{T}}(\ell)} r^{s(v)} \sqrt{\log \Delta_{\mathcal{T}}(v)}-2 C r^{s(z)+1} \\
& \geqslant \operatorname{val}_{r}(\mathcal{T}, s)-2 C r^{s(z)+1} \\
& \geqslant \frac{1}{2} \operatorname{val}_{r}(\mathcal{T}, s) .
\end{aligned}
$$

where in the second line we have used property (1) of Definition 3.8, in the third line, we have used $r \geqslant 4$, and in the final line we have used our assumption that $\operatorname{val}_{r}(\mathcal{T}, s) \geqslant 4 C r^{s(z)+1}$.

It remains to prove that $\operatorname{val}_{r}(\mathcal{T}, s) \geqslant \operatorname{val}_{r}\left(\mathcal{T}^{\prime}, s^{\prime}\right)$. The issue here is that it is possible $\mathcal{L}^{\prime} \subsetneq \mathcal{L}$. However, by our choice of $u$ at each stage (as in equation (45)), it is guaranteed that $\ell \in \mathcal{L}^{\prime}$ for a certain $\ell \in \mathcal{L}$ such that $\operatorname{val}_{r}(\mathcal{T}, s)=\sum_{v \in \mathcal{P}(\ell)} r^{s(v)} \sqrt{\log \Delta(v)}$. This completes the proof. 
We next study the subtrees of separated trees. In what follows, we continue denoting by $\left.s\right|_{\mathcal{T}^{\prime}}$ the restriction of $s$ on $\mathcal{T}^{\prime}$ for $\mathcal{T}^{\prime} \subseteq \mathcal{T}$, and we use a subscript $\mathcal{T}^{\prime}$ to refer to the subtree $\mathcal{T}^{\prime}$.

Lemma 3.12. For every $r$-separated tree $(\mathcal{T}, s)$, there is a subtree $\mathcal{T}^{\prime} \subseteq \mathcal{T}$ such that $\left(\mathcal{T}^{\prime},\left.s\right|_{\mathcal{T}^{\prime}}\right)$ is an $r$-separated tree satisfying the following conditions.

1. $\operatorname{val}_{r}(\mathcal{T}, s) \asymp \operatorname{val}_{r}\left(\mathcal{T}^{\prime},\left.s\right|_{\mathcal{T}^{\prime}}\right)$.

2. For every $v \in \mathcal{T}^{\prime} \backslash \mathcal{L}_{\mathcal{T}^{\prime}}, \Delta_{\mathcal{T}^{\prime}}(v)=\Delta(v)$.

3. For every $v \in \mathcal{T}^{\prime} \backslash \mathcal{L}_{\mathcal{T}^{\prime}}$ and $w \in \mathcal{L}_{\mathcal{T}^{\prime}} \cap \mathcal{T}_{v}$,

$$
\sum_{u \in \mathcal{P}(v, w)} r^{s(u)} \sqrt{\log \Delta_{\mathcal{T}^{\prime}}(u)} \geqslant \frac{1}{2} r^{s(p(v))} \sqrt{\log \Delta_{\mathcal{T}^{\prime}}(p(v))}
$$

Proof. We construct the subtree $\mathcal{T}^{\prime}$ in the following way. We examine the vertices of $v \in \mathcal{T}$ in the breadth-first search order (that is, we order the vertices such that their distances to the root are non-decreasing). If $v$ is not deleted yet and for some $\ell \in \mathcal{L} \cap \mathcal{T}_{v}$,

$$
\sum_{u \in \mathcal{P}(v, \ell)} r^{s(u)} \sqrt{\log \Delta_{\mathcal{T}}(u)} \leqslant r^{s(p(v))} \sqrt{\log \Delta_{\mathcal{T}}(p(v))},
$$

we delete all the descendants of $v$. Let $\mathcal{T}^{\prime}$ be the subtree obtained at the end of the process. It is clear that $\left(\mathcal{T}^{\prime},\left.s\right|_{\mathcal{T}^{\prime}}\right)$ is a separated tree, and it remains to verify the required properties.

By the construction of our subtree $\mathcal{T}^{\prime}$, we see that whenever a vertex is deleted, all its siblings are deleted. So for a node $v \in \mathcal{T}^{\prime} \backslash \mathcal{L}_{\mathcal{T}^{\prime}}$, all the children in $\mathcal{T}$ of $v$ are preserved in $\mathcal{T}^{\prime}$, yielding property (2).

Note that if $v \in \mathcal{L}_{\mathcal{T}^{\prime}} \backslash \mathcal{L}$, there exists $\ell \in \mathcal{L} \cap \mathcal{T}_{v}$ such that (47) holds. Therefore, we see

$$
\sum_{u \in \mathcal{P}(z, v)} r^{s(u)} \sqrt{\log \Delta_{\mathcal{T}^{\prime}}(u)}=\sum_{u \in \mathcal{P}(z, v) \backslash\{v\}} r^{s(u)} \sqrt{\log \Delta_{\mathcal{T}}(u)} \geqslant \frac{1}{2} \sum_{u \in \mathcal{P}(z, \ell)} r^{s(u)} \sqrt{\log \Delta_{\mathcal{T}}(u)} \geqslant \frac{1}{2} \operatorname{val}_{r}(\mathcal{T}, s) .
$$

This verifies property (1) (noting that the reverse inequality is trivial).

Take $v \in \mathcal{T}^{\prime} \backslash \mathcal{L}_{\mathcal{T}^{\prime}}$ and $w \in \mathcal{L}_{\mathcal{T}^{\prime}} \cap \mathcal{T}_{v}$. If $w \in \mathcal{L}$, we see that (46) holds for $v$ and $w$ since (47) does not hold for $v$ and $\ell=w$ (otherwise all the descendants of $v$ have to be deleted and $v$ will be a leaf node in $\mathcal{T}^{\prime}$ ). If $w \notin \mathcal{L}$, there exists $\ell_{0} \in \mathcal{L} \cap \mathcal{T}_{w}$ such that

$$
\sum_{u \in \mathcal{P}\left(w, \ell_{0}\right)} r^{s(u)} \sqrt{\log \Delta_{\mathcal{T}}(u)} \leqslant r^{s(p(w))} \sqrt{\log \Delta_{\mathcal{T}}(p(w))} .
$$

Recall that (47) fails with $\ell=\ell_{0}$. Altogether, we conclude that

$$
\begin{aligned}
\sum_{u \in \mathcal{P}(v, w)} r^{s(u)} \sqrt{\log \Delta_{\mathcal{T}^{\prime}}(u)} & =\sum_{u \in \mathcal{P}\left(v, \ell_{0}\right)} r^{s(u)} \sqrt{\log \Delta_{\mathcal{T}}(u)}-\sum_{u \in \mathcal{P}\left(w, \ell_{0}\right)} r^{s(u)} \sqrt{\log \Delta_{\mathcal{T}}(u)} \\
& \geqslant \frac{1}{2} \sum_{u \in \mathcal{P}\left(v, \ell_{0}\right)} r^{s(u) \sqrt{\log \Delta_{\mathcal{T}}(u)}} \\
& \geqslant \frac{1}{2} r^{s(p(v))} \sqrt{\log \Delta_{\mathcal{T}}(p(v))}
\end{aligned}
$$

establishing property (3) and completing the proof. 
Finally, we observe that separated trees are stable in the following sense.

Lemma 3.13. Fix $0<\delta<1$. Suppose that $(\mathcal{T}, s)$ is an $r$-separated tree in $X$, and for every node $v \in V$, we delete all but $\lceil\delta \cdot|\Gamma(v)|\rceil$ of its children. Denote by $\mathcal{T}^{\prime}$ the induced tree on the connected component containing $z(\mathcal{T})$. Then $\left(\mathcal{T}^{\prime},\left.s\right|_{\mathcal{T}^{\prime}}\right)$ is an r-separated tree and

$$
\operatorname{val}_{r}(\mathcal{T}, s) \asymp_{\delta} \operatorname{val}_{r}\left(\mathcal{T}^{\prime},\left.s\right|_{\mathcal{T}^{\prime}}\right) .
$$

Proof. It is clear that Properties (1), (2) and (3) of separated trees are preserved for the induced tree $\mathcal{T}^{\prime}$ for $\left.s\right|_{\mathcal{T}^{\prime}}$. So $\left(\mathcal{T}^{\prime}, s\right)$ is an $r$-separated tree. Furthermore, for every leaf $\ell$ of $\mathcal{T}^{\prime}$,

$$
\begin{aligned}
\sum_{v \in \mathcal{P}(\ell)} r^{s(v)} \sqrt{\log \Delta_{\mathcal{T}^{\prime}}(v)} & \geqslant \sum_{v \in \mathcal{P}(\ell)} r^{s(v)} \sqrt{\log (1+\lceil\delta \cdot|\Gamma(v)|\rceil)} \\
& \geqslant c(\delta) \sum_{v \in \mathcal{P}(\ell)} r^{s(v)} \sqrt{\log (1+|\Gamma(v)|)} \geqslant c(\delta) \operatorname{val}_{r}(\mathcal{T}, s)
\end{aligned}
$$

where $c(\delta)$ is a constant depending only on $\delta$. It follows that $\operatorname{val}_{r}\left(\mathcal{T}^{\prime},\left.s\right|_{\mathcal{T}^{\prime}}\right) \geqslant c(\delta) \operatorname{val}_{r}(\mathcal{T}, s)$, completing the proof since the reverse direction is obvious.

\subsection{Computing an approximation to $\gamma_{2}$ deterministically}

We now present a deterministic algorithm for computing an approximation to $\gamma_{2}$.

Theorem 3.14. Let $(X, d)$ be a finite metric space, with $n=|X|$. If, for any two points $x, y \in X$, one can compute $d(x, y)$ in time polynomial in $n$, then one can compute a number $A(X, d)$ in polynomial time, for which

$$
A(X, d) \asymp \gamma_{2}(X, d)
$$

Proof. Fix $r \geqslant 16$. First, let us assume that $1 \leqslant d(x, y) \leqslant r^{M}$ for $x \neq y \in X$ and some $M \in \mathbb{N}$. Fix $x_{0} \in X$.

Our algorithm constructs functions $\varphi_{0}, \varphi_{1}, \ldots, \varphi_{M}: X \rightarrow \mathbb{R}_{+}$. We will return the value $A(X, d)=\varphi_{M}\left(x_{0}\right)$. First put $\varphi_{1}(x)=\varphi_{0}(x)=0$ for all $x \in X$. Next, we show how to construct $\varphi_{j}$ given $\varphi_{0}, \varphi_{1}, \ldots, \varphi_{j-1}$.

For $x \in X$ and $r \geqslant 0$, we use $B(x, r) \triangleq\{y \in X: d(x, y) \leqslant r\}$. First, we construct a maximal $\frac{1}{3} r^{j-1}$ net $N_{j}$ in $X$ in the following way. Supposing that $y_{1}, \ldots, y_{k}$ have already been chosen, let $y_{k+1}$ be a point satisfying

$$
\varphi_{j-2}\left(y_{k+1}\right)=\max \left\{\varphi_{j-2}(y): y \in X \backslash \bigcup_{i=1}^{k} B\left(x, \frac{1}{3} r^{j-1}\right)\right\},
$$

as long as there exists some point of $X \backslash \bigcup_{i=1}^{k} B\left(x, \frac{1}{3} r^{j-1}\right)$ remaining. For $x \in X$, set

$$
g_{j}(x)=y_{\min \left\{k: d\left(x, y_{k}\right) \leqslant \frac{1}{3} r^{j-1}\right\}} \cdot
$$

Now we define $\varphi_{j}(x)$ for $x \in X$. Suppose that $B\left(x, 2 r^{j}\right) \cap N_{j}=\left\{y_{\ell_{1}}, y_{\ell_{2}}, \ldots, y_{\ell_{h}}\right\}$, with $\ell_{1} \leqslant$ $\ell_{2} \leqslant \cdots \leqslant \ell_{h}$, and define 
I. $\varphi_{j}(x)=\varphi_{j-1}(x)$ if $B\left(g_{j}(x), 4 r^{j}\right) \backslash B\left(g_{j}(x), \frac{1}{16} r^{j-2}\right)$ is empty.

II. Otherwise,

$$
\varphi_{j}(x)=\max \left\{\max _{k \leqslant h}\left(r^{j} \sqrt{\log k}+\min _{i \leqslant k} \varphi_{j-2}\left(y_{\ell_{i}}\right)\right), \max \left\{\varphi_{j-1}(z): z \in B\left(x, \frac{1}{3} r^{j-1}\right)\right\}\right\} .
$$

Now, we verify that $\left\{\varphi_{j}\right\}_{j=0}^{M}$ satisfies the conditions of Theorem 3.3. The monotonicity condition (1) is satisfied by construction. We will now verify condition (2), starting with the following lemma.

Lemma 3.15. For any $j \geqslant 0$, If $d(s, t) \leqslant r^{j}$ and $B\left(g_{j}(s), 4 r^{j}\right) \backslash B\left(g_{j}(s), \frac{1}{16} r^{j-2}\right)$ is empty, then $\varphi_{j}(s)=\varphi_{j}(t)$.

Proof. We prove this by induction on $j$. Clearly it holds vacuously for $j \leqslant 2$. Assume that it holds for $\varphi_{0}, \varphi_{1}, \ldots, \varphi_{j-1}$ and $j \geqslant 2$. By the condition of the lemma and the fact that $s \in B\left(g_{j}(s), \frac{1}{3} r^{j-1}\right)$, we have

$$
d\left(s, g_{j}(s)\right) \leqslant \frac{1}{16} r^{j-2},
$$

which implies that $B\left(s, 2 r^{j}\right) \backslash B\left(s, \frac{1}{8} r^{j-2}\right)$ is also empty. Furthermore, we have $g_{j}(s)=g_{j}(t)$, since otherwise $d\left(g_{j}(s), g_{j}(t)\right) \geqslant \frac{1}{3} r^{j-1}$, and we would conclude that

$$
2 r^{j} \geqslant d\left(g_{j}(t), s\right) \geqslant d\left(g_{j}(s), g_{j}(t)\right)-d\left(s, g_{j}(s)\right) \geqslant \frac{1}{3} r^{j-1}-\frac{1}{16} r^{j-2} \geqslant \frac{1}{8} r^{j-1},
$$

contradicting the fact that $B\left(s, 2 r^{j}\right) \backslash B\left(s, \frac{1}{8} r^{j-2}\right)$ is empty. It follows that

$$
B\left(s, 2 r^{j}\right) \backslash B\left(s, \frac{1}{8} r^{j-2}\right)=\emptyset \text { and } B\left(t, 2 r^{j}\right) \backslash B\left(t, \frac{1}{8} r^{j-2}\right)=\emptyset .
$$

Since $g_{j}(s)=g_{j}(t)$, we conclude that both $\varphi_{j}(s)$ and $\varphi_{j}(t)$ are defined by case (I) above, hence

$$
\varphi_{j}(s)=\varphi_{j-1}(s) \text { and } \varphi_{j}(t)=\varphi_{j-1}(t) .
$$

So we are done by induction unless $B\left(g_{j}(s), 4 r^{j-1}\right) \backslash B\left(g_{j}(s), \frac{1}{16} r^{j-3}\right)$ is non-empty, in which case $\varphi_{j-1}(s)$ and $\varphi_{j-1}(t)$ are defined by case (II). But from (50) and $d(s, t) \leqslant r^{j}$, we see that $B\left(t, 2 r^{j-1}\right)=B\left(s, 2 r^{j-1}\right)$ and $B\left(s, \frac{1}{3} r^{j-2}\right)=B\left(t, \frac{1}{3} r^{j-2}\right)$ as well. This implies that $\varphi_{j-1}(s)$ and $\varphi_{j-1}(t)$ see the same maximization in (48), hence $\varphi_{j-1}(s)=\varphi_{j-1}(t)$ and by (51) we are done.

Now, let $s, t_{1}, \ldots, t_{N} \in X$ be as in condition (2), and let $B\left(s, 2 r^{j}\right) \cap N_{j}=\left\{y_{\ell_{1}}, y_{\ell_{2}}, \ldots, y_{\ell_{h}}\right\}$ be such that $\ell_{1} \leqslant \ell_{2} \leqslant \cdots \leqslant \ell_{h}$. If $B\left(g_{j}(s), 4 r^{j}\right) \backslash B\left(g_{j}(s), \frac{1}{16} r^{j-1}\right)$ is empty, then $N=1$, and Lemma 3.15 implies that $\varphi_{j}(s)=\varphi_{j}\left(t_{1}\right) \geqslant \varphi_{j-2}\left(t_{1}\right)$, where the latter inequality follows from monotonicity. Thus we may assume that $\varphi_{j}(s)$ is defined by case (II).

To every $t_{i}$, we can associate a distinct point $g_{j}\left(t_{i}\right) \in B\left(s, 2 r^{j}\right) \cap N_{j}$, and by construction we have $\varphi_{j-2}\left(g_{j}\left(t_{i}\right)\right) \geqslant \varphi_{j-2}\left(t_{i}\right)$, since $\varphi_{j-2}\left(y_{k}\right)$ is decreasing as $k$ increases. Using this property again in conjunction with the definition (48), we have

$$
\begin{aligned}
\varphi_{j}(s) & \geqslant r^{j} \sqrt{\log N}+\min \left\{\varphi_{j-2}\left(y_{\ell_{i}}\right): i=1, \ldots, N\right\} \\
& \geqslant r^{j} \sqrt{\log N}+\min \left\{\varphi_{j-2}\left(g_{j}\left(t_{i}\right)\right): i=1, \ldots, N\right\} \\
& \geqslant r^{j} \sqrt{\log N}+\min \left\{\varphi_{j-2}\left(t_{i}\right): i=1, \ldots, N\right\},
\end{aligned}
$$


completing our verification of condition (2) of Theorem 3.3. Applying Theorem 3.3, we see that

$$
\gamma_{2}(X, d) \lesssim \sup _{x \in X, i \in \mathbb{Z}} \varphi_{i}(x)=\varphi_{M}\left(x_{0}\right)=A(X, d)
$$

To prove the matching lower bound, we first build a tree $\mathcal{T}$ whose vertex set is a subset of $X \times \mathbb{Z}$. The root of $\mathcal{T}$ is $\left(x_{0}, M\right)$. In general, if $(x, j)$ is already a vertex of $\mathcal{T}$ with $j \geqslant 1$, then we add children to $(x, j)$ according to the maximizer of (48). If $\varphi_{j}(x)=\varphi_{j-1}(z)$, then we make $(z, j-1)$ the only child of $(x, j)$. Otherwise, we put the nodes $\left(y_{1}, j-2\right), \ldots,\left(y_{h}, j-2\right)$ as children of $(x, j)$, where $\left\{y_{i}\right\} \subseteq N_{j}$ are the nodes that achieve the maximum in (48).

Let the pair $\left(\mathcal{T}^{\prime}, s\right)$ be a constructed in the following way from $\mathcal{T}$. We replace every maximal path of the form $\left(x, j_{0}\right),\left(x, j_{0}-1\right), \ldots,\left(x, j_{0}-k\right)$ by the vertex $x$ and put $s(x)=j_{0}-k$. It follows immediately by construction that

$$
\operatorname{val}_{r}\left(\mathcal{T}^{\prime}, s\right) \lesssim \varphi_{M}\left(x_{0}\right)+r \operatorname{diam}(X, d) \lesssim \varphi_{M}\left(x_{0}\right),
$$

where the latter inequality follows from (52), since $\varphi_{M}\left(x_{0}\right) \gtrsim \gamma_{2}(X, d) \gtrsim \operatorname{diam}(X, d)$. Note that the correction term of $\operatorname{diam}(X, d)$ in (53) is simply because of the use of $\Delta(v)=|\Gamma(v)|+1$ in the definition (43).

We next build a $\left(1, r, 8, \frac{1}{16}\right)$-tree $\mathcal{F}$, which essentially captures the structure of the tree $\mathcal{T}$. In general, the sets in $\mathcal{F}$ will be balls in $X$, with the node $(x, j) \in \mathcal{T}$ being associated with the set $B\left(x, 4 r^{j}\right)$ in $\mathcal{F}$, which will have label $n\left(B\left(x, 4 r^{j}\right)\right)=j$.

We construct the $\left(1, r, 8, \frac{1}{16}\right)$-tree $\mathcal{F}$ recursively. The root of $\mathcal{F}$ is $B\left(x_{0}, 4 r^{M}\right)$ (which is equal to $X)$, and we define $n\left(B\left(x, 4 r^{j}\right)\right)=M$. In general, if $\mathcal{F}$ contains the set $B\left(x, 4 r^{j}\right)$ corresponding to the node $(x, j) \in \mathcal{T}$, and if $(x, j)$ has children $\left(y_{1}, j-2\right),\left(y_{2}, j-2\right), \ldots,\left(y_{h}, j-2\right) \in \mathcal{T}$, we add the sets $B\left(y_{i}, 4 r^{j-2}\right)$ as children of $B\left(x, 4 r^{j}\right)$ in $\mathcal{F}$, with $n\left(B\left(y_{i}, 4 r^{j-2}\right)\right)=j-2$. Likewise, if $(z, j-1)$ is the child of $(x, j)$, then we add the set $B\left(z, 4 r^{j-1}\right)$ as the unique child of $B\left(x, 4 r^{j}\right)$ in $\mathcal{F}$ and put $n\left(B\left(z, 4 r^{j-1}\right)\right)=j-1$. We continue in this manner until $\mathcal{T}$ is exhausted.

We now verify that $\mathcal{F}$ is indeed a $\left(1, r, 8, \frac{1}{6}\right)$-tree. First, note that if $(z, j-1)$ is a child of $(x, j)$ in $\mathcal{T}$, then clearly $B\left(z, 4 r^{j-1}\right) \subseteq B\left(x, 4 r^{j}\right)$ since this can only happen if $d(x, z) \leqslant \frac{1}{3} r^{j-1}$. Also, if $\left(y_{1}, j-2\right), \ldots,\left(y_{h}, j-2\right)$ are the children of $(x, j)$, then by the construction of the maps in (48), we have $d\left(y_{i}, x\right) \leqslant 2 r^{j}$, hence $B\left(y_{i}, 4 r^{j-2}\right) \subseteq B\left(x, 4 r^{j}\right)$, recalling that $r \geqslant 16$. Furthermore, for $i \neq k$, since $y_{i}, y_{k} \in N_{j}$, we have $d\left(y_{i}, y_{k}\right) \geqslant \frac{1}{3} r^{j-1}$, so $B\left(y_{i}, 4 r^{j-2}\right) \cap B\left(y_{k}, 4 r^{j-2}\right)=\emptyset$, verifying that $\mathcal{F}$ is indeed a tree of subsets. In fact, we have the estimate

$$
d\left(B\left(y_{i}, 4 r^{j-2}\right), B\left(y_{k}, 4 r^{j-2}\right)\right) \geqslant \frac{1}{3} r^{j-1}-8 r^{j-2} \geqslant \frac{1}{6} r^{j-1}=\frac{1}{6} r^{n\left(B\left(x, 4 r^{j}\right)\right)-1},
$$

using $r \geqslant 16$. This verifies that property $(2)$ of a $\left(1, r, 1, \frac{1}{6}\right)$-tree is satisfied. Furthermore, property (1) of a $\left(1, r, 8, \frac{1}{6}\right)$-tree follows immediately by construction. Finally, to verify property (3), note that for any set in our tree of subsets $\mathcal{F}$, corresponding to a node of the form $(x, j) \in \mathcal{T}$, we have $\operatorname{diam}\left(B\left(x, 4 r^{j}\right)\right) \leqslant 8 r^{j}$ and $n\left(B\left(x, 4 r^{j}\right)\right)=j$.

By construction, we have

$$
\operatorname{val}_{r}\left(\mathcal{T}^{\prime}, s\right) \lesssim \operatorname{size}_{r}(\mathcal{F})+r \operatorname{diam}(X, d),
$$

and Lemma 3.7 yields $\gamma_{2}(X, d) \gtrsim \operatorname{size}_{r}(\mathcal{F})+\operatorname{diam}(X, d)\left(\operatorname{using} \gamma_{2}(X, d) \gtrsim \operatorname{diam}(X, d)\right)$. Combining this with (53) shows that

$$
\gamma_{2}(X, d) \gtrsim \operatorname{val}_{r}\left(\mathcal{T}^{\prime}, s\right) \gtrsim \varphi_{M}\left(x_{0}\right)=A(X, d)
$$


Together with (52), this shows that $\gamma_{2}(X, d) \asymp A(X, d)$.

The only thing left is to remove the dependence of our running time on $M$. But since there are at most $n^{2}$ distinct distances in $(X, d)$, only $O\left(n^{2}\right)$ of the maps $\varphi_{0}, \varphi_{1}, \ldots, \varphi_{M}$ are distinct. More precisely, suppose that there is no pair $u, v \in X$ satisfying $d(u, v) \in\left[r^{j-3}, r^{j+1}\right]$ for some $j \in \mathbb{Z}$. In that case, $\varphi_{j}(x)$ is defined by case (I) for all $x \in X$, and thus $\varphi_{j} \equiv \varphi_{j-1}$. Obviously, we may skip computation of the intermediate non-distinct maps (and it is easy to see which maps to skip by precomputing the values of $j$ such that there are $u, v \in X$ with $d(u, v) \in\left[r^{j-3}, r^{j+1}\right]$.) Since there are only $O\left(n^{2}\right)$ non-trivial values of $j$, this completes the proof.

\subsection{Tree-like properties of the Gaussian free field}

Finally, we consider how the resistance metric (and hence the Gaussian free field) allows us to obtain trees with special properties. Consider a network $G(V)$, and the associated metric space $\left(V, \sqrt{R_{\text {eff }}}\right)$. Let $(\mathcal{T}, s)$ be an $r$-separated tree in $G$. We say that $(\mathcal{T}, s)$ is strongly $r$-separated if, for every non-root node $v \in \mathcal{T}$, we have the inequality

$$
\sqrt{R_{\mathrm{eff}}\left(v, \mathcal{T} \backslash \mathcal{T}_{v}\right)} \geqslant \frac{1}{20} r^{s(p(v))-1}
$$

where $p(v)$ denotes the parent of $v$ in $\mathcal{T}$.

Lemma 3.16. For any network $G(V)$ and any $r \geqslant 96$, let $\left(\mathcal{T}_{0}, s\right)$ be an arbitrary $r$-separated tree on the space $\left(V, \sqrt{R_{\text {eff }}}\right)$. Then there is an induced strongly $r$-separated tree $(\mathcal{T}, s)$ such that $\left|\Gamma_{\mathcal{T}}(v)\right| \geqslant\left|\Gamma_{\mathcal{T}_{0}}(v)\right| / 2$ for all $v \in \mathcal{T} \backslash \mathcal{L}_{\mathcal{T}}$. Furthermore

$$
\operatorname{val}_{r}(\mathcal{T}, s) \asymp \operatorname{val}_{r}\left(\mathcal{T}_{0}, s\right) .
$$

Proof. Consider any non-leaf node $v \in \mathcal{T}_{0}$ with children $c_{1}, \ldots, c_{k}$, where $k \geqslant 1$. If $k=1$, let $S_{v}=\left\{c_{1}\right\}$. Otherwise, we wish to apply Proposition 2.10 to the sets $\left\{\mathcal{T}_{c_{i}}\right\}_{i=1}^{k}$. By property (2) of separated trees, we get that for all $x \in \mathcal{T}_{c_{i}}, y \in \mathcal{T}_{c_{j}}$ with $i \neq j$

$$
R_{\mathrm{eff}}(x, y) \geqslant\left(\frac{1}{2} r^{s(v)-1}\right)^{2}=\frac{1}{4} r^{2(s(v)-1)} .
$$

Combined with property (3) of separated trees, Proposition 2.10 yields that there exists a subset $S_{v} \subseteq\left\{c_{1}, \ldots, c_{k}\right\}$ with $\left|S_{v}\right| \geqslant k / 2$ such that for $c \in S_{v}$, we have

$$
R_{\text {eff }}\left(\mathcal{T}_{c}, \mathcal{T}_{v} \backslash\left(\mathcal{T}_{c} \cup\{v\}\right)\right) \geqslant \frac{1}{4} r^{2(s(v)-1)} \cdot \frac{1}{24} \geqslant \frac{1}{96} r^{2(s(v)-1)} .
$$

Applying Lemma 2.13 with $A=\mathcal{T}_{c}, B_{1}=\mathcal{T}_{v} \backslash\left(\mathcal{T}_{c} \cup\{v\}\right)$ and $B_{2}=\{v\}$, we get that

$$
R_{\text {eff }}\left(\mathcal{T}_{c}, \mathcal{T}_{v} \backslash \mathcal{T}_{c}\right) \geqslant \frac{1}{100} r^{2(s(v)-1)}
$$

Next, consider the induced $r$-separated tree $(\mathcal{T}, s)$ that arises from deleting, for every non-leaf node $v \in \mathcal{T}_{0}$, all the children not in $S_{v}$ as well as all their descendants. It is clear that for all $v \in \mathcal{T} \backslash \mathcal{L}_{\mathcal{T}}$, we have $\left|\Gamma_{\mathcal{T}}(v)\right| \geqslant\left|\Gamma_{\mathcal{T}_{0}}(v)\right| / 2$. Lemma 3.13 then yields that

$$
\operatorname{val}_{r}(\mathcal{T}, s) \asymp \operatorname{val}_{r}\left(\mathcal{T}_{0}, s\right) .
$$


It remains to verify that $(\mathcal{T}, s)$ is strongly $r$-separated. Define $D_{0}=1$ and for $h \geqslant 1$,

$$
D_{h}=D_{h-1}\left(1-D_{h-1}^{2} r^{-4 h}\right) .
$$

It is straightforward to verify that $D_{h} \geqslant 1 / 2$ for all $h \geqslant 0$, since $r \geqslant 2$.

We now prove, by induction on the height of $\mathcal{T}$, that for every node $u$ at $\operatorname{depth} h \geqslant 1$ in $\mathcal{T}$,

$$
\sqrt{R_{\text {eff }}\left(u, \mathcal{T} \backslash \mathcal{T}_{u}\right)} \geqslant \frac{1}{10} r^{s(p(u))-1} D_{h-1} .
$$

By the preceding remarks, this verifies (54), completing the proof of the lemma.

Let $z=z(\mathcal{T})$ be the root, and let $v$ be some child of $z$. Let $u \in \mathcal{T}_{v}$ be a node at depth $h$ in $\mathcal{T}_{v}$ (and hence at depth $h+1$ in $\mathcal{T}$ ). By (56), we have

$$
\sqrt{R_{\text {eff }}\left(u, \mathcal{T} \backslash \mathcal{T}_{v}\right)} \geqslant \sqrt{R_{\text {eff }}\left(\mathcal{T}_{v}, \mathcal{T} \backslash \mathcal{T}_{v}\right)} \geqslant \frac{1}{10} r^{s(p(v))-1}
$$

If $u=v$, then the preceding inequality yields (57). Otherwise, $u \neq v$, and $h \geqslant 1$.

By the induction hypothesis (57) applied to $u$ and $\mathcal{T}_{v}$, we have

$$
\sqrt{R_{\mathrm{eff}}\left(u, \mathcal{T}_{v} \backslash \mathcal{T}_{u}\right)} \geqslant \frac{1}{10} r^{s(p(u))-1} D_{h-1}
$$

Since $u \in \mathcal{T}_{v}$ is a node at depth $h$, we get from property (1) of a separated tree that $s(p(v)) \geqslant$ $s(p(u))+2 h$ and therefore

$$
\frac{1}{10} r^{s(p(u))-1} D_{h-1} \leqslant r^{-2 h} \cdot \frac{1}{10} r^{s(p(v))-1} D_{h-1} .
$$

Now, using (58) and (59), we apply Lemma 2.13 with $A=\{u\}, B_{1}=\mathcal{T}_{v} \backslash \mathcal{T}_{u}$ and $B_{2}=\mathcal{T} \backslash \mathcal{T}_{v}$, yielding

$$
\begin{aligned}
\sqrt{R_{\mathrm{eff}}\left(u, \mathcal{T} \backslash \mathcal{T}_{u}\right)} & \geqslant \frac{\frac{1}{10} r^{s(p(u))-1} D_{h-1} \cdot \frac{1}{10} r^{s(p(v))-1}}{\sqrt{\left(\frac{1}{10} r^{s(p(u))-1} D_{h-1}\right)^{2}+\left(\frac{1}{10} r^{s(p(v))-1}\right)^{2}}} \\
& \geqslant \frac{1}{10} r^{s(p(u))-1} D_{h-1} \frac{1}{\sqrt{1+\left(D_{h-1} r^{-2 h}\right)^{2}}} \\
& \geqslant \frac{1}{10} r^{s(p(u))-1} D_{h-1}\left(1-D_{h-1}^{2} r^{-4 h}\right),
\end{aligned}
$$

where the second transition follows from (60) and the third transition follows from the fact that $\left(1+x^{2}\right)^{-1 / 2} \geqslant 1-x^{2}$. This completes the proof.

Good trees inside the GFF. Consider a Gaussian free field $\left\{\eta_{x}\right\}_{x \in V}$ corresponding to network $G(V)$ with the associated metric space $(V, d)$, where $d(x, y)=\left(\mathbb{E}\left(\eta_{x}-\eta_{y}\right)^{2}\right)^{1 / 2}$.

Proposition 3.17. For some $r_{0} \geqslant 2$ and any $r \geqslant r_{0}$ and $C \geqslant 1$, there exists a constant $K=$ $K(C, r)$ depending only on $C$ and $r$ such that the following holds. For an arbitrary Gaussian free field $\left\{\eta_{x}\right\}_{x \in V}$ with $\gamma_{2}(V, d) \geqslant K \operatorname{diam}(V)$, there exists an $r$-separated tree $(\mathcal{T}, s)$ with set of leaves $\mathcal{L}$, such that the following properties hold. 
(a) $\operatorname{val}_{r}(\mathcal{T}, s) \asymp_{r, C} \gamma_{2}(X, d)$.

(b) For every $v \in V$, $\operatorname{dist}_{L^{2}}\left(\eta_{v}, \operatorname{aff}\left(\left\{\eta_{u}\right\}_{u \notin \mathcal{T}_{v}}\right)\right) \geqslant \frac{1}{20} r^{s(p(v))-1}$.

(c) For every $v \in V, \Delta(v) \geqslant \exp \left(C^{2} r^{2} 4^{s(z)-s(v)}\right)$ for all $v \in \mathcal{T} \backslash \mathcal{L}$.

(d) For every $v \in \mathcal{T} \backslash \mathcal{L}$ and $w \in \mathcal{L} \cap \mathcal{T}_{v}$,

$$
\sum_{u \in \mathcal{P}(v, w)} r^{s(u)} \sqrt{\log \Delta(u)} \geqslant \frac{1}{2} r^{s(p(v))} \sqrt{\log \Delta(p(v))} .
$$

We call such a tree $\mathcal{T}$ a $C$-good r-separated tree.

Proof. By definition of the GFF, we have $d=\sqrt{R_{\text {eff }}}$ for some network $G(V)$. Applying Theorem 3.9, there exists an $r$-separated tree $\left(\mathcal{T}_{0}, s_{0}\right)$ such that $\operatorname{val}_{r}\left(\mathcal{T}_{0}, s_{0}\right) \asymp_{r} \gamma_{2}(V, d)$.

Recalling property (3) of Definition 3.8 and the assumption that $\gamma_{2}(V, d) \geqslant K \operatorname{diam}(V)$, we can then select $K$ large enough such that the condition of Lemma 3.11 is satisfied for the separated tree $\left(\mathcal{T}_{0}, s_{0}\right)$. Then applying Lemma 3.11 , we can get a $2 C$-regular separated tree $\left(\mathcal{T}_{1}, s_{1}\right)$ with $\operatorname{val}_{r}\left(\mathcal{T}_{1}, s_{1}\right) \asymp_{r, C} \operatorname{val}_{r}\left(\mathcal{T}_{0}, s_{0}\right)$.

At this point, using Lemma 3.16, we obtain a $C$-regular strongly $r$-separated tree $\left(\mathcal{T}_{2}, s_{2}\right)$ such that $\operatorname{val}_{r}\left(\mathcal{T}_{2}, s_{2}\right) \asymp_{r} \gamma_{2}(V, d)$. That is to say, the tree $\left(\mathcal{T}_{2}, s_{2}\right)$ satisfies properties (a) and (c). Furthermore, by Lemma 2.15, we see that property (b) holds for $\left(\mathcal{T}_{2}, s_{2}\right)$ because it is equivalent to the strongly $r$-separated property (54).

Finally, Lemma 3.12 implies that there exists a subtree $\mathcal{T} \subseteq \mathcal{T}_{2}$ with $\operatorname{val}_{r}\left(\mathcal{T}, s_{2} \mid \mathcal{T}\right) \asymp_{r, C}$ $\operatorname{val}_{r}\left(\mathcal{T}_{2}, s_{2}\right)$ such that property (d) holds for $\mathcal{T}$ and properties (a) and (c) are preserved (note that by property (2) of Lemma 3.12, the degrees of non-leaf nodes are preserved). Observe that property (b) is preserved by taking subtrees. Writing $s=\left.s_{2}\right|_{\mathcal{T}}$, we conclude that the separated tree $(\mathcal{T}, s)$ satisfies all the required properties, completing the proof.

\section{The cover time}

We now turn to our main theorem.

Theorem 4.1. For any network $G(V)$ with total conductance $\mathcal{C}=\sum_{x \in V} c_{x}$, we have

$$
t_{\mathrm{cov}}^{\circlearrowright}(G) \asymp \mathcal{C}\left[\gamma_{2}\left(V, \sqrt{R_{\mathrm{eff}}}\right)\right]^{2} .
$$

Combined with Theorem 2.3, this also yields a positive answer to the strong conjecture of Winkler and Zuckerman [54].

Corollary 4.2. For every $\delta \in(0,1)$, for any network $G(V)$ with total conductance $\mathcal{C}=\sum_{x \in V} c_{x}$,

$$
t_{\mathrm{cov}}^{\circlearrowright}(G) \asymp \mathcal{C}\left[\gamma_{2}\left(V, \sqrt{R_{\text {eff }}}\right)\right]^{2} \asymp_{\delta} t_{\mathrm{bl}}(G, \delta) .
$$

For the remainder of this section, we denote

$$
\mathfrak{S}=\gamma_{2}\left(V, \sqrt{R_{\mathrm{eff}}}\right) .
$$


It is clear that for all $0<\delta<1$, we have $t_{\mathrm{cov}}^{\circlearrowright}(G) \leqslant t_{\mathrm{bl}}(G, \delta)$, and $t_{\mathrm{bl}}(G, \delta) \lesssim \delta \mathcal{C S}^{2}$ by Theorem 2.3 , Thus, in order to prove the preceding corollary and Theorem 4.1, we need only show that

$$
t_{\text {cov }}^{\circlearrowright}(G) \gtrsim \mathcal{C} \mathfrak{S}^{2} .
$$

Let $\left\{W_{t}\right\}$ be the continuous-time random walk on $G(V)$, and let $\left\{L_{t}^{v}\right\}_{v \in V}$ be the local times, as defined in Section 2. Applying the isomorphism theorem (Theorem 1.14) with some fixed $v_{0} \in V$, we have

$$
\left\{L_{\tau(t)}^{x}+\frac{1}{2} \eta_{x}^{2}: x \in V\right\} \stackrel{\text { law }}{=}\left\{\frac{1}{2}\left(\eta_{x}+\sqrt{2 t}\right)^{2}: x \in V\right\},
$$

for some associated Gaussian process $\left\{\eta_{x}\right\}_{x \in V}$. By Lemma 2.14, this process is a Gaussian free field, and we have for every $x, y \in V$,

$$
d(x, y) \triangleq \sqrt{\mathbb{E}\left|\eta_{x}-\eta_{y}\right|^{2}}=\sqrt{R_{\mathrm{eff}}(x, y)}
$$

Let $\mathfrak{D}=\max _{x, y \in V} d(x, y)$ be the diameter of the Gaussian process.

Proof outline. Let $\{\mathfrak{L}>0\}$ be the event $\left\{L_{\tau(t)}^{x}>0: x \in V\right\}$. Consider a set $S \subseteq \mathbb{R}^{V}$, and let $S_{L}$ and $S_{R}$ be the events corresponding to the left and right-hand sides of (63) falling into $S$. Our goal is to find such a set $S$ so that for some $t \asymp \mathfrak{S}^{2}$, we have

$$
\mathbb{P}\left(S_{R}\right)-\mathbb{P}\left(S_{L} \cap\{\mathfrak{L}>0\}\right) \geqslant c,
$$

for some universal constant $c>0$. In this case, with probability at least $c$, the set of uncovered vertices $\left\{v: L_{\tau(t)}^{v}=0\right\}$ is non-empty. Using the fact that the inverse local time $\tau(t)$ is $\gtrsim \mathcal{C} t$ with probability at least $1-c / 2$, we will conclude that $t_{\text {cov }}^{\circlearrowright}(G) \gtrsim \mathfrak{C S}^{2}$.

Thus we are left to give a lower bound on $\mathbb{P}\left(S_{R}\right)$ and an upper bound on $\mathbb{P}\left(S_{L} \cap\{\mathfrak{L}>0\}\right)$. Since the structure of the local times process $\left\{L_{t}^{x}\right\}$ conditioned on $\{\mathfrak{L}>0\}$ can be quite unwieldy, we will only use first moment bounds for the latter task. Calculating a lower bound on $\mathbb{P}\left(S_{R}\right)$ will require a significantly more delicate application of the second-moment method, but here we will be able to exploit the full power of Gaussian processes and the majorizing measures theory.

Before defining the set $S \subseteq \mathbb{R}^{V}$, we describe it in broad terms. By (64) and Theorem (MM), we know that for some $t_{0} \asymp \mathfrak{S}^{2}$, we should have $\mathbb{E} \inf _{x \in V} \eta_{x}=-\mathbb{E} \sup _{x \in V} \eta_{x}$ close to $-\sqrt{2 t_{0}}$. By Lemma 2.2, we know that the standard deviation of $\inf _{x \in V} \eta_{x}$ is $O(\mathfrak{D})$. Thus we can expect that with probability bounded away from 0 , for the right choice of $t_{0} \asymp \mathfrak{S}^{2}$, some value on the right-hand side of (63) is $O(\mathfrak{D})$ for $t=t_{0}$.

Now, when $\mathbb{E} \sup _{x \in V} \eta_{x} \gg \mathfrak{D}$, it is intuitively true that for $t=\varepsilon t_{0}$ and $\varepsilon>0$ small, there should be many points $x \in V$ with $\eta_{x} \approx-\sqrt{2 t}$. If these points have some level of independence, then we should expect that with probability bounded away from 0 , there is some $x \in V$ with $\left|\eta_{x}-\sqrt{2 t}\right|$ very small (much smaller than $O(\mathfrak{D})$ ). Our set $S$ will represent the existence of such a point. On the other hand, we will argue that if all the local times $\left\{L_{\tau(t)}^{x}\right\}$ are positive, then the probability for the left-hand side to have such a low value is small. 


\subsection{A tree-like sub-process}

First, observe that by the commute time identity, $t_{\mathrm{cov}}^{\circlearrowright}(G) \geqslant \mathcal{C} \max _{x, y \in V} R_{\text {eff }}(x, y)=\mathcal{C} \mathfrak{D}^{2}$. Thus in proving Theorem 4.1, we may assume that

$$
\mathfrak{S} \geqslant K \mathfrak{D}
$$

for any universal constant $K \geqslant 1$. In particular, by an application of Proposition 3.17, we can assume the existence of an $r$-separated tree $(\mathcal{T}, s)$ in $(V, d)$, for some fixed $r \geqslant 128$, with root $z=v_{0}$, and such that for some constant $C \geqslant 1$ and $\theta=\theta(C)$, properties (67), (70), (71), and (72) below are satisfied. We will choose $C$ sufficiently large later, independent of any other parameters.

For each $u \in \mathcal{T}$, let $h_{u}$ denote the height of $u$, where we order the tree so that $h_{z}=0$, where $z$ is the root. Recalling that $\mathcal{L}$ is the set of leaves of $\mathcal{T}$, for each $v \in \mathcal{L}$, let

$$
\mathcal{P}(v)=\left\{f_{v}(0), f_{v}(1), \ldots, f_{v}\left(h_{v}\right)\right\}
$$

be the set of nodes on the path from $z=f_{v}(0)$ to $v=f_{v}\left(h_{v}\right)$, where $f_{v}(i)$ is the parent of $f_{v}(i+1)$, for $0 \leqslant i<h$. First, we can require that for every $v \in \mathcal{L}$,

$$
\sigma_{v} \geqslant \frac{1}{\theta} \mathfrak{S}
$$

where

$$
\begin{aligned}
\chi_{v}(k) & \triangleq r^{s\left(f_{v}(k)\right)} \sqrt{\log \Delta\left(f_{v}(k)\right)}, \\
\sigma_{v} & \triangleq \sum_{k=0}^{h_{v}-1} \chi_{v}(k) .
\end{aligned}
$$

Furthermore, we can require that the tree $\mathcal{T}$ satisfies, for every $v \in V$,

$$
\sum_{i=j+1}^{h_{v}-1} \chi_{v}(i) \geqslant C \cdot 2^{j} \cdot r^{s\left(f_{v}(j)\right)}
$$

as well as

$$
\Delta\left(f_{v}(k)\right) \geqslant \exp \left(C^{2} r^{2} 4^{k}\right)
$$

Finally, we require that for every $v \in \mathcal{T}$,

$$
\operatorname{dist}_{L^{2}}\left(\eta_{v}, \operatorname{aff}\left(\left\{\eta_{u}\right\}_{u \notin \mathcal{T}_{v}}\right)\right) \geqslant \frac{1}{20} r^{s(p(v))-1} .
$$

All these requirements are justified by Proposition 3.17 .

The distinguishing event. For $u, v \in \mathcal{L}$, we let $h_{u v}$ be the height of the least common ancestor of $u$ and $v$. We will use $\operatorname{deg}_{\downarrow}(v)=|\Gamma(v)|$ to denote the number of children of $v$. Define

$$
m_{u}=\prod_{k=0}^{h_{u}-1} \operatorname{deg}_{\downarrow}\left(f_{u}(k)\right), \text { and } m_{u v}=\prod_{k=0}^{h_{u v}-1} \operatorname{deg}_{\downarrow}\left(f_{u}(k)\right) \text {. }
$$


First, we fix

$$
\varepsilon=\frac{1}{2^{10} r \theta}
$$

For every $v \in \mathcal{L}$, consider the events

$$
\mathcal{E}_{v}(\varepsilon)=\left\{\left|\eta_{v}-\varepsilon \mathfrak{S}\right| \leqslant 50 r^{s(p(v))} m_{v}^{-3 / 4}\right\} .
$$

Instead of arguing directly about the events $\mathcal{E}_{v}(\varepsilon)$, we will couple them to leaf events of a "percolation" process on $\mathcal{T}$. In particular, in Section 4.2, we will prove the following lemma.

Lemma 4.3. For all $v \in \mathcal{L}$, there exist events $\mathcal{E}_{v}$ such that the following properties hold.

1. $\mathcal{E}_{v} \subseteq \mathcal{E}_{v}(\varepsilon)=\left\{\left|\eta_{v}-\varepsilon \mathfrak{S}\right| \leqslant 50 r^{s(p(v))} m_{v}^{-3 / 4}\right\}$.

2. $\mathbb{P}\left(\mathcal{E}_{v}\right) \geqslant \frac{1}{2} m_{v}^{-7 / 8}$.

3. $\mathbb{P}\left(\mathcal{E}_{u} \cap \mathcal{E}_{v}\right) \leqslant m_{u v}^{1 / 8}\left(m_{u} m_{v}\right)^{-7 / 8}$.

In Section 4.3, we will prove that for any events $\left\{\mathcal{E}_{v}\right\}_{v \in \mathcal{L}}$ satisfying properties (2) and (3) of Lemma 4.3, we have

$$
\mathbb{P}\left(\bigcup_{u \in \mathcal{L}} \mathcal{E}_{u}\right) \geqslant \frac{1}{8}
$$

Thus for $t=\frac{1}{2} \varepsilon^{2} \mathfrak{S}^{2}$, we have

$$
\mathbb{P}\left(\exists v \in V: \frac{1}{2}\left(\eta_{v}+\sqrt{2 t}\right)^{2} \leqslant 50^{2} r^{2 s(p(v))} m_{v}^{-3 / 2}\right) \geqslant \frac{1}{8} .
$$

In light of the discussion surrounding (65), the reader should think of

$$
S=\left\{s \in \mathbb{R}^{V}: s_{v} \leqslant 50^{2} r^{2 s(p(v))} m_{v}^{-3 / 2} \text { for some } v \in V\right\},
$$

and then (177) gives the desired lower bound on $\mathbb{P}\left(S_{R}\right)$. We now turn to an upper bound on $\mathbb{P}\left(S_{L} \cap\{\mathfrak{L}>0\}\right)$. The next lemma is proved in Section 4.4.

Lemma 4.4. For $t \geqslant \frac{1}{2} \varepsilon^{2} \mathfrak{S}^{2}$,

$$
\mathbb{P}\left(\bigcup_{v \in \mathcal{L}}\left\{0<L_{\tau(t)}^{v} \leqslant 50^{2} \cdot r^{2 s(p(v))} m_{v}^{-3 / 2}\right\}\right) \leqslant \frac{1}{16} .
$$

From (178) and (77), we conclude that with probability at least $1 / 16$, we must have $L_{\tau(t)}^{v}=0$ for some $v \in V$ and $t=\frac{1}{2} \varepsilon^{2} \mathfrak{S}^{2}$, else (63) is violated. This implies that

$$
\mathbb{P}_{v_{0}}\left(\tau_{\text {cov }}^{\circlearrowright} \geqslant \tau\left(\frac{1}{2} \varepsilon^{2} \mathfrak{S}^{2}\right)\right) \geqslant \frac{1}{16} .
$$

To finish our proof of (62) and complete the proof of Theorem 4.1, we will apply Lemma 2.7 with $\beta=\frac{1}{96}$. In particular, we may choose $K=96 / \varepsilon$ in (66), and then applying Lemma 2.7 yields

$$
\mathbb{P}\left(\tau\left(\frac{1}{2} \varepsilon^{2} \mathfrak{S}^{2}\right) \leqslant \mathcal{C} \frac{\varepsilon^{2} \mathfrak{S}^{2}}{192}\right) \leqslant \frac{1}{32} .
$$


Combining this with (79) yields

$$
\mathbb{P}_{v_{0}}\left(\tau_{\text {cov }}^{\circlearrowright} \geqslant \mathcal{C} \frac{\varepsilon^{2} \mathfrak{S}^{2}}{192}\right) \geqslant \frac{1}{16}
$$

In particular, $\tau_{\operatorname{cov}}^{\circlearrowright} \gtrsim \mathcal{C} \varepsilon^{2} \mathfrak{S}^{2}$. This completes the proof of (62), and hence of Theorem 4.1 .

\subsection{The coupling}

The present section is devoted to the proof of Lemma 4.3. Toward this end, we will try to find a leaf $v \in \mathcal{L}$ for which $\eta_{v} \approx \varepsilon \mathfrak{S}$. As in Lemma 4.3(1), the level of closeness we desire is gauged according to a proper scale, $r^{s(p(v))}$, as well as to the number of other leaves we expect to see at this scale, which is represented roughly by $m_{v}^{-3 / 4}$ (the value $3 / 4$ is not essential here, and any other value in $(1 / 2,1)$ would suffice).

Our goal is to find a such a leaf by starting at the root of the tree, and arguing that some of its children should be somewhat close to the target $\varepsilon \mathfrak{S}$. This closeness is achieved using the fact that, by definition of an $r$-separated tree, the children are separated in the Gaussian distance, and thus exhibit some level of independence. We will continue in this manner inductively, arguing that the children which are somewhat close to the target have their own children which we could expect to be even closer, and so on. We aim to shrink these windows around the target more and more so they are small enough once we reach the leaves. There are a number of difficulties involved in executing this scheme. In particular, conditioning on the exact values of the children of the root could determine the entire process, making future levels moot. Thus we must first select a careful filtering which allows us to reserve some randomness for later levels. This is done in Section 4.2.1,

Furthermore, the intermediate targets have to be arranged according to the variances along the root-leaf paths in our tree. This corresponds to the fact that, although we have a uniform lower bound on each $\sigma_{v}$ (from (67)), the summation defining the $\sigma_{v}$ 's could put different weights on the various levels (recall (69) ). The targets also have to take into account random "noise" from the filter described above, and thus the targets themselves must be random. This "window analysis" is performed in Section 4.2.2.

\subsubsection{Restructuring the randomness}

We know that $\eta_{z}=0$, since $z=v_{0}$ is the root of $\mathcal{T}$ (and the starting point of the associated random walk). Fix a depth-first ordering of $\mathcal{T}$ (one starts at the root and explores as far as possible along each branch before backtracking). Write $u \prec v$ if $u$ is explored before $v$, and $u \preceq v$ if $u \prec v$ or $u=v$. For $u \neq z$, we write $u^{-}$for the vertex preceding $u$ in the DFS order. Let $\mathcal{F}=\operatorname{span}\left(\left\{\eta_{x}: x \in \mathcal{T}\right\}\right)$. For a node $v \in \mathcal{T}$, let $\mathcal{F}_{v}=\operatorname{span}\left(\left\{\eta_{u}\right\}_{u \preceq v}\right)$ and $\mathcal{F}_{v}^{-}=\operatorname{span}\left(\left\{\eta_{u}\right\}_{u \prec v}\right)$. We next associate a centered Gaussian process $\left\{\xi_{x}: x \in \mathcal{T}\right\}$ to $\left\{\eta_{x}: x \in \mathcal{T}\right\}$ in the following inductive way. Define $\xi_{z}=0$. Now, assuming we have defined $\xi_{u}$ for $u \prec v$, we define $\xi_{v}$ by writing

$$
\eta_{v}=\zeta_{v}+\xi_{v}
$$

where $\zeta_{v} \in \mathcal{F}_{v^{-}}$and $\xi_{v} \perp \mathcal{F}_{v^{-}}$. Observe that, by construction, $\left\{\xi_{u}\right\}_{u \preceq v}$ forms an orthogonal basis in $L^{2}$ for $\mathcal{F}_{v}$.

Applying (72), we have for all $u \in \mathcal{T}$,

$$
\left\|\xi_{u}\right\|_{2}=\operatorname{dist}_{L^{2}}\left(\eta_{u}, \operatorname{span}\left(\left\{\eta_{w}\right\}_{w \prec u}\right)\right) \geqslant \operatorname{dist}_{L^{2}}\left(\eta_{u}, \operatorname{span}\left(\left\{\eta_{w}\right\}_{w \notin \mathcal{T}_{u}}\right)\right) \geqslant \frac{1}{20} r^{s(p(u))-1},
$$


where we used the fact that the span and the affine hull are the same since $\xi_{z}=0$. For $v \in \mathcal{L}$, define the subspaces

$$
\begin{aligned}
& \mathcal{F}_{v, k}=\operatorname{span}\left(\left\{\xi_{u}: f_{v}(k) \prec u \preceq f_{v}(k+1)\right\}\right), \\
& \mathcal{F}_{v, k}^{-}=\operatorname{span}\left(\left\{\xi_{u}: f_{v}(k) \prec u \prec f_{v}(k+1)\right\}\right) .
\end{aligned}
$$

For $0 \leqslant k \leqslant h_{v}-1$, define inductively $\tilde{\eta}_{v, 0}=0$, and

$$
\tilde{\eta}_{v, k+1}=\tilde{\eta}_{v, k}+\operatorname{proj}_{\mathcal{F}_{v, k}}\left(\eta_{v}\right)
$$

Note that the subspaces $\left\{\mathcal{F}_{v, k}\right\}_{k=0}^{h_{v}}$ are mutually orthogonal, and together they span $\mathcal{F}_{v}$. Thus,

$$
\tilde{\eta}_{v, h_{v}}=\eta_{v} .
$$

Furthermore, by the definition of the subspace $\mathcal{F}_{v, k}$, we can decompose

$$
\tilde{\eta}_{v, k+1}-\tilde{\eta}_{v, k}=\tilde{\zeta}_{v, k}+\tilde{\xi}_{v, k},
$$

where $\tilde{\zeta}_{v, k} \in \mathcal{F}_{v, k}^{-}$, and $\tilde{\xi}_{v, k} \perp \mathcal{F}_{v, k}^{-}$. The next lemma states that $\tilde{\xi}_{v, k}$ has at least comparable variance to $\tilde{\zeta}_{v, k}$.

Lemma 4.5. For every $v \in \mathcal{L}$ and $k=0,1, \ldots, h_{v}-1$, we have the estimates

$$
\left\|\tilde{\zeta}_{v, k}\right\|_{2} \leqslant 8 r^{s\left(f_{v}(k)\right)}
$$

and,

$$
\frac{1}{64} r^{s\left(f_{v}(k)\right)-1} \leqslant\left\|\tilde{\xi}_{v, k}\right\|_{2} \leqslant 8 r^{s\left(f_{v}(k)\right)} .
$$

Proof. Writing the telescoping sum,

$$
\eta_{v}=\sum_{j=0}^{h_{v}-1} \eta_{f_{v}(j+1)}-\eta_{f_{v}(j)}
$$

we see that

$$
\left\|\operatorname{proj}_{\mathcal{F}_{v, k}}\left(\eta_{v}\right)\right\|_{2} \leqslant \sum_{j=k}^{h_{v}-1}\left\|\eta_{f_{v}(j+1)}-\eta_{f_{v}(j)}\right\|_{2} \leqslant \sum_{j=k}^{h_{v}-1} 4 r^{s\left(f_{v}(j)\right)} \leqslant 8 r^{s\left(f_{v}(k)\right)},
$$

where we used properties (1) and (3) of the separated tree, and have assumed $r \geqslant 2$.

Thus by orthogonality and (83), we have

$$
\left\|\tilde{\zeta}_{v, k}\right\|_{2} \leqslant\left\|\tilde{\eta}_{v, k+1}-\tilde{\eta}_{v, k}\right\|_{2}=\left\|\operatorname{proj}_{\mathcal{F}_{v, k}}\left(\eta_{v}\right)\right\|_{2} \leqslant 8 r^{s\left(f_{v}(k)\right)}
$$

and precisely the same conclusion holds for $\tilde{\xi}_{v, k}$. 
Next, we establish a lower bound on $\left\|\tilde{\xi}_{v, k}\right\|_{2}$. From (81) and (83),

$$
\begin{aligned}
\tilde{\xi}_{v, k}= & \operatorname{proj}_{\mathcal{F}_{v, k}}\left(\eta_{v}\right)-\operatorname{proj}_{\mathcal{F}_{v, k}^{-}}\left(\eta_{v}\right) \\
= & \sum_{j=k}^{h_{v}-1}\left(\operatorname{proj}_{\mathcal{F}_{v, k}}\left(\eta_{f_{v}(j+1)}-\eta_{f_{v}(j)}\right)-\operatorname{proj}_{\mathcal{F}_{v, k}^{-}}\left(\eta_{f_{v}(j+1)}-\eta_{f_{v}(j)}\right)\right) \\
= & {\left[\operatorname{proj}_{\mathcal{F}_{v, k}}\left(\eta_{f_{v}(k+1)}-\eta_{f_{v}(k)}\right)-\operatorname{proj}_{\mathcal{F}_{v, k}^{-}}\left(\eta_{f_{v}(k+1)}-\eta_{f_{v}(k)}\right)\right] } \\
& +\sum_{j=k+1}^{h_{v}-1}\left(\operatorname{proj}_{\mathcal{F}_{v, k}}\left(\eta_{f_{v}(j+1)}-\eta_{f_{v}(j)}\right)-\operatorname{proj}_{\mathcal{F}_{v, k}^{-}}\left(\eta_{f_{v}(j+1)}-\eta_{f_{v}(j)}\right)\right) .
\end{aligned}
$$

Observe that the term in brackets is precisely

$$
\operatorname{proj}_{\mathcal{F}_{v, k}}\left(\eta_{f_{v}(k+1)}\right)-\operatorname{proj}_{\mathcal{F}_{v, k}^{-}}\left(\eta_{f_{v}(k+1)}\right)=\xi_{f_{v}(k+1)},
$$

since $\eta_{f_{v}(k)} \perp \mathcal{F}_{v, k}$. In particular, we arrive at

$$
\begin{aligned}
\left\|\tilde{\xi}_{v, k}\right\|_{2} & \geqslant\left\|\xi_{f_{v}(k+1)}\right\|_{2}-\sum_{j=k+1}^{h_{v}-1}\left\|\eta_{f_{v}(j+1)}-\eta_{f_{v}(j)}\right\|_{2} \\
& \geqslant \frac{1}{32} r^{s\left(f_{v}(k)\right)-1}-2 r^{s\left(f_{v}(k+1)\right)} \\
& \geqslant \frac{1}{32} r^{s\left(f_{v}(k)\right)-1}-2 r^{s\left(f_{v}(k)\right)-2} \\
& \geqslant \frac{1}{64} r^{s\left(f_{v}(k)\right)-1}
\end{aligned}
$$

where in the second line we have used (80) and properties (1) and (2) of the separated tree, and in the final line we have used $r \geqslant 128$.

\subsubsection{Defining the events $\mathcal{E}_{v}$}

Recall that our goal now is to find many leaves $v \in \mathcal{L}$ with $\eta_{v} \approx \varepsilon \mathfrak{S}$. Now, writing

$$
\eta_{v}=\sum_{k=0}^{h_{v}-1} \operatorname{proj}_{\mathcal{F}_{v, k}}\left(\eta_{v}\right)=\sum_{k=0}^{h_{v}-1}\left(\tilde{\zeta}_{v, k}+\tilde{\xi}_{v, k}\right),
$$

our "ideal" goal would be to hit a window around the target by getting the $k$ th term of this sum close to

$$
a_{v}(k) \triangleq \varepsilon \mathfrak{S} \frac{\chi_{v}(k)}{\sigma_{v}},
$$

for $k=0,1, \ldots, h_{v}-1$. We will use the variance of the $\tilde{\xi}_{v, k}$ variables (recall Lemma 4.5) to lower bound the probability that some points get closer to the desired target. On the other hand, we will treat the $\tilde{\zeta}_{v, k}$ variables as noise which has to be bounded in absolute value.

This noise cannot always be countered in a single level, but it can be countered on average along the path to the leaf; this is the content of (70). We will amortize this cost over future targets as follows. Let $b_{v}(0)=0$ and for $k=0,1, \ldots, h_{v}-2$, define

$$
\begin{aligned}
\rho_{v}(k) & =\tilde{\zeta}_{v, k}+\tilde{\xi}_{v, k}-a_{v}(k)+b_{v}(k), \\
b_{v}(k+1) & =\sum_{i=0}^{k} \frac{\chi_{v}(k+1)}{\sum_{\ell=i+1}^{h_{v}-1} \chi_{v}(\ell)} \rho_{v}(i) .
\end{aligned}
$$


Clearly $\rho_{v}(0)=\tilde{\zeta}_{v, 0}+\tilde{\xi}_{v, 0}-a_{v}(0)$ represents how much we miss our first target. A similar fact holds for the final target, as the next lemma argues; in between, the errors are spread out proportional to the contribution to $\operatorname{val}_{r}(\mathcal{T}, s)$ for each of the the remaining levels (represented by the $\chi_{v}(k)$ values). Here $b_{v}(k)$ represents the error that is meant to be absorbed in the $k$-th level.

Lemma 4.6. For every $v \in \mathcal{L}$,

$$
\rho_{v}\left(h_{v}-1\right)=\eta_{v}-\varepsilon \mathfrak{S}
$$

Proof. We have,

$$
\sum_{k=0}^{h_{v}-2} b_{v}(k+1)=\sum_{k=0}^{h_{v}-2} \sum_{i=0}^{k} \frac{\chi_{v}(k+1)}{\sum_{\ell=i+1}^{h_{v}-1} \chi_{v}(\ell)} \rho_{v}(i)=\sum_{i=0}^{h_{v}-2} \rho_{v}(i) \sum_{k=i}^{h_{v}-2} \frac{\chi_{v}(k+1)}{\sum_{\ell=i+1}^{h_{v}-1} \chi_{v}(\ell)}=\sum_{i=0}^{h_{v}-2} \rho_{v}(i) .
$$

Also note that

$$
\sum_{k=0}^{h_{v}-1} \rho_{v}(k)=\sum_{k=0}^{h_{v}-1}\left(\tilde{\zeta}_{v, k}+\tilde{\xi}_{v, k}-a_{v}(k)+b_{v}(k)\right)=\eta_{v}-\varepsilon \mathfrak{S}+\sum_{k=0}^{h_{v}-1} b_{v}(k) .
$$

Combined with $b_{v}(0)=0$ and (88), it follows that $\rho_{v}\left(h_{v}-1\right)=\eta_{v}-\varepsilon \mathfrak{S}$, completing the proof.

We now define the events

$$
\begin{aligned}
& \mathcal{A}_{v}(k)=\left\{\left|\tilde{\zeta}_{v, k}\right| \leqslant \varepsilon \theta \chi_{v}(k)\right\}, \\
& \mathcal{B}_{v}(k)=\left\{\left|\rho_{v}(k)\right| \leqslant w_{v}(k)\right\},
\end{aligned}
$$

where, for $0 \leqslant k \leqslant h_{v}-2, w_{v}(k)$ is selected so that

$$
\mathbb{P}\left(\mathcal{B}_{v}(k) \mid \tilde{\zeta}_{v, k}+b_{v}(k)\right)=\operatorname{deg}_{\downarrow}\left(f_{v}(k)\right)^{-1 / 8} .
$$

We emphasize that the windown $w_{v}(k)$ is not deterministic. And, for $k=h_{v}-1$, we select $w_{v}(k)$ so that

$$
\mathbb{P}\left(\mathcal{B}_{v}(k) \mid \tilde{\zeta}_{v, k}+b_{v}(k)\right)=\operatorname{deg}_{\downarrow}\left(f_{v}(k)\right)^{-1 / 8} m_{v}^{-3 / 4},
$$

Remark 2. Here, $w_{v}(k)$ can be thought to represent the window size around the random target. The value of $w_{v}(k)$ is chosen to make the probabilities in (89) and (90) exact, allowing us to couple seamlessly to the percolation process in Section 4.3. The key fact, proved in Lemma 4.7, is that the window sizes actually satisfy a deterministic upper bound, assuming that all the "good" events on the path from the root to $f_{v}(k)$ occurred. Thus one should think of the true window size as the bounds specified in (94) and (95), while the random value is for the purpose of the coupling.

For $0 \leqslant k \leqslant \ell \leqslant h_{v}-1$, define

$$
\mathcal{A}_{v}(k, \ell) \triangleq \bigcap_{i=k}^{\ell} \mathcal{A}_{v}(i) \text { and } \mathcal{B}_{v}(k, \ell) \triangleq \bigcap_{i=k}^{\ell} \mathcal{B}_{v}(i)
$$

Since $\tilde{\xi}_{v, k} \in \sigma\left(\mathcal{F}_{v, k} \backslash \mathcal{F}_{v, k}^{-}\right)$(see, e.g. (87)), we see that the event $\mathcal{B}_{v}(k)$ is conditionally independent of $\sigma\left(\mathcal{F}_{f_{v}(k+1)}^{-}\right)$given the value of $\tilde{\zeta}_{v, k}+b_{v}(k)$. This implies that for all events $\mathcal{E}_{0} \in \sigma\left(\mathcal{F}_{f_{v}(k+1)}^{-}\right)$such that $\mathcal{E}_{0} \cap \mathcal{A}_{v}(0, k) \cap \mathcal{B}_{v}(0, k-1) \neq \emptyset$,

$$
\mathbb{P}\left(\mathcal{B}_{v}(k) \mid \mathcal{A}_{v}(0, k), \mathcal{B}_{v}(0, k-1), \mathcal{E}_{0}\right)= \begin{cases}\operatorname{deg}_{\downarrow}\left(f_{v}(k)\right)^{-1 / 8}, & \text { if } 0 \leqslant k<h_{v}-1, \\ \operatorname{deg}_{\downarrow}\left(f_{v}(k)\right)^{-1 / 8} m_{v}^{-3 / 4}, & \text { if } k=h_{v}-1 .\end{cases}
$$


Finally, for $v \in \mathcal{L}$, we define the event

$$
\mathcal{E}_{v}=\mathcal{A}_{v}\left(0, h_{v}-1\right) \cap \mathcal{B}_{v}\left(0, h_{v}-1\right) .
$$

Window analysis. We will now show that our final window $w_{v}\left(h_{v}-1\right)$ is small enough. Observe that our choice of $w_{v}(k)$ is not deterministic. Nevertheless, we will give an absolute upper bound. The bound is essentially the natural one: For any node $u$ in the tree, and any child $v$ of $u$, the standard deviation of $\eta_{u}-\eta_{v}$ is $O\left(r^{s(u)}\right)$. This follows from property (3) of the $r$-separated tree (recall Definition 3.8).

Lemma 4.7. For every $v \in \mathcal{L}$ and $k=0,1, \ldots, h_{v}-2$, if $\mathcal{A}_{v}(0, k)$ and $\mathcal{B}_{v}(0, k-1)$ hold then,

$$
w_{v}(k) \leqslant 50 r^{s\left(f_{v}(k)\right)} \text {. }
$$

Furthermore, if $\mathcal{A}_{v}\left(0, h_{v}-1\right)$ and $\mathcal{B}_{v}\left(0, h_{v}-2\right)$ hold, then

$$
w_{v}\left(h_{v}-1\right) \leqslant 50 r^{s\left(f_{v}\left(h_{v}-1\right)\right)} m_{v}^{-3 / 4} .
$$

Proof. For $k=0$, we have $\rho_{v}(0)=\tilde{\zeta}_{v, 0}+\tilde{\xi}_{v, 0}-a_{v}(0)$. By (67), we have

$$
a_{v}(0)=\varepsilon \mathfrak{S} \chi_{v}(0) / \sigma_{v} \leqslant \theta \varepsilon \chi_{v}(0)=\theta \varepsilon r^{s\left(f_{v}(0)\right)} \sqrt{\log \Delta\left(f_{v}(0)\right)} .
$$

Furthermore, from Lemma 4.5, we know that for all $k \geqslant 0$,

$$
\frac{1}{64} r^{s\left(f_{v}(k)\right)-1} \leqslant\left\|\tilde{\xi}_{v, k}\right\|_{2} \leqslant 8 r^{s\left(f_{v}(k)\right)} .
$$

Now, consider a value $w>0$ such that

$$
w \leqslant a_{v}(0)+\varepsilon \theta \chi_{v}(0) \leqslant 2 \theta \varepsilon r^{s\left(f_{v}(0)\right)} \sqrt{\log \Delta\left(f_{v}(0)\right)} .
$$

Using (97) and recalling the Gaussian density, we have

$$
\begin{aligned}
\mathbb{P}\left(\left|\rho_{v}(0)\right| \leqslant w \mid \mathcal{A}_{v}(0)\right) & \geqslant \mathbb{P}\left(\left|\rho_{v}(0)\right| \leqslant w \mid \tilde{\zeta}_{v, 0}=-\varepsilon \theta \chi_{v}(0)\right) \\
& =\mathbb{P}\left(\left|\tilde{\xi}_{v, 0}-a_{v}(0)-\varepsilon \theta \chi_{v}(0)\right| \leqslant w\right) \\
& \geqslant \frac{1}{2} \frac{w}{\sqrt{2 \pi} 8 r^{s\left(f_{v}(0)\right)}} \exp \left(-\frac{1}{2}(128 \varepsilon r \theta)^{2} \log \Delta\left(f_{v}(0)\right)\right) \\
& =\frac{w}{16 \sqrt{2 \pi} r^{s\left(f_{v}(0)\right)}} \Delta\left(f_{v}(0)\right)^{-\frac{1}{2}(128 \varepsilon r \theta)^{2}}
\end{aligned}
$$

Recalling the assumption (71), we have $\sqrt{\log \Delta\left(f_{v}(0)\right)} \geqslant C r \geqslant 16 \sqrt{2 \pi} 2^{10} r$, by choosing $C$ large enough. In particular,

$$
\varepsilon \theta \chi_{v}(0) \geqslant\left(16 \sqrt{2 \pi} 2^{10} \varepsilon \theta r\right) r^{s\left(f_{v}(0)\right)}=16 \sqrt{2 \pi} r^{s\left(f_{v}(0)\right)},
$$

recalling (74). Thus setting $w=16 \sqrt{2 \pi} r^{s\left(f_{v}(0)\right)}$ satisfies (98), and applying (99) we have

$$
\mathbb{P}\left(\left|\rho_{v}(0)\right| \leqslant 16 \sqrt{2 \pi} r^{s\left(f_{v}(0)\right)} \mid \mathcal{A}_{v}(0)\right) \geqslant \Delta\left(f_{v}(0)\right)^{-\frac{1}{2}(128 \varepsilon r \theta)^{2}} \geqslant \operatorname{deg}_{\downarrow}\left(f_{v}(0)\right)^{-1 / 8},
$$


where we have used $\frac{1}{2}(128 \varepsilon r \theta)^{2}=\frac{1}{128}$, and $\Delta\left(f_{v}(0)\right) \geqslant 16$ from (171). Therefore

$$
w_{v}(0) \leqslant 16 \sqrt{2 \pi} r^{s\left(f_{v}(0)\right)} \leqslant 50 r^{s\left(f_{v}(0)\right)},
$$

recalling the definition of $w_{v}(0)$ from (89)).

Now suppose that (94) holds for all $k \leqslant \ell<h_{v}-2$, and consider the case $k=\ell+1$. If the events $\left\{\mathcal{B}_{v}(j): 0 \leqslant j \leqslant \ell\right\}$ hold, then

$$
\left|\rho_{v}(j)\right| \leqslant w_{v}(j) \leqslant 50 r^{s\left(f_{v}(j)\right)},
$$

where the first inequality is from the definition of $\mathcal{B}_{v}(j)$, and the second is from the induction hypothesis. Using (70), it follows that

$$
\left|b_{v}(k)\right| \leqslant \sum_{i=0}^{k-1} \frac{\chi_{v}(k)}{\sum_{\ell=i+1}^{h_{v}-1} \chi_{v}(\ell)}\left|\rho_{v}(i)\right| \leqslant \frac{2}{C} \chi_{v}(k) .
$$

Recall that $\rho_{v}(k)=\tilde{\zeta}_{v, k}+\tilde{\xi}_{v, k}-a_{v}(k)+b_{v}(k)$. Similar to the $k=0$ case, we obtain that for

$$
0<w \leqslant 2 \theta \varepsilon r^{s\left(f_{v}(k)\right)} \sqrt{\log \Delta\left(f_{v}(k)\right)},
$$

we have,

$$
\begin{gathered}
\mathbb{P}\left(\left|\rho_{v}(k)\right| \leqslant w \mid \mathcal{A}_{v}(i), \mathcal{B}_{v}(i) \text { for all } 0 \leqslant i<k, \mathcal{A}_{v}(k)\right) \\
\geqslant \mathbb{P}\left(\left|\tilde{\xi}_{v, k}-a_{v}(k)-\varepsilon \theta \chi_{v}(k)-\frac{2}{C} \chi_{v}(k)\right| \leqslant w\right) \\
\geqslant \frac{1}{2} \frac{w}{\sqrt{2 \pi} 8 r^{s\left(f_{v}(k)\right)}} \Delta\left(f_{v}(k)\right)^{-\frac{1}{2}(128 r)^{2}\left(\varepsilon \theta+C^{-1}\right)^{2}} .
\end{gathered}
$$

Now, by choosing $C \geqslant 1024 r$, and recalling (74), we see that

$$
\frac{1}{2}(128 r)^{2}\left(\varepsilon \theta+C^{-1}\right)^{2} \leqslant \frac{1}{32} .
$$

Since $\Delta\left(f_{v}(k)\right) \geqslant 16$ (again, by (71) $)$, we conclude that

$$
\mathbb{P}\left(\left|\rho_{v}(k)\right| \leqslant 16 \sqrt{2 \pi} r^{s\left(f_{v}(k)\right)} \mid \mathcal{A}_{v}(i), \mathcal{B}_{v}(i) \text { for all } 0 \leqslant i<k, \mathcal{A}_{v}(k)\right) \geqslant \operatorname{deg}_{\downarrow}\left(f_{v}(k)\right)^{-1 / 8}
$$

This implies $w_{v}(k) \leqslant 16 \sqrt{2 \pi} r^{s\left(f_{v}(k)\right)} \leqslant 50 r^{s\left(f_{v}(k)\right)}$, where we recall once again the definition of $w_{v}(k)$ from (89).

An almost identical argument yields that $w_{v}\left(h_{v}-1\right) \leqslant 50 r^{s\left(f_{v}\left(h_{v}-1\right)\right)} m_{v}^{-3 / 4}$.

The next lemma states that the events $\mathcal{E}_{v}$ as defined in (93) satisfy requirement (1) of Lemma 4.3 .

Lemma 4.8. If $\mathcal{E}_{v}$ occurs, then

$$
\left|\eta_{v}-\varepsilon \mathfrak{S}\right| \leqslant w_{v}\left(h_{v}-1\right) \leqslant 50 r^{s\left(f_{v}\left(h_{v}-1\right)\right)} m_{v}^{-3 / 4}
$$

Proof. This follows directly from Lemma 4.6, the identity (82) and the definition of $\mathcal{B}_{v}(k)$. 
The first moment. We now give lower bounds on the probability of the event $\mathcal{E}_{v}$.

Lemma 4.9. For every $v \in \mathcal{L}$,

$$
\mathbb{P}\left(\mathcal{E}_{v}\right) \geqslant \frac{1}{2} m_{v}^{-7 / 8}
$$

Proof. We have,

$$
\begin{aligned}
\mathbb{P}\left(\mathcal{E}_{v}\right) & =\prod_{k=0}^{h_{v}-1} \mathbb{P}\left(\mathcal{A}_{v}(k) \mid \mathcal{A}_{v}(0, k-1), \mathcal{B}_{v}(0, k-1)\right) \mathbb{P}\left(\mathcal{B}_{v}(k) \mid \mathcal{A}_{v}(0, k), \mathcal{B}_{v}(0, k-1)\right) \\
& =m_{v}^{-3 / 4} \prod_{k=0}^{h_{v}-1} \operatorname{deg}_{\downarrow}\left(f_{v}(k)\right)^{-1 / 8} \prod_{k=0}^{h_{v}-1} \mathbb{P}\left(\mathcal{A}_{v}(k) \mid \mathcal{A}_{v}(0, k-1), \mathcal{B}_{v}(0, k-1)\right) \\
& =m_{v}^{-7 / 8} \prod_{k=0}^{h_{v}-1} \mathbb{P}\left(\mathcal{A}_{v}(k)\right),
\end{aligned}
$$

where the second line follows from (92), and the third line from the fact that $\mathcal{A}_{v}(k)$ is independent of $\left\{\mathcal{A}_{v}(i), \mathcal{B}_{v}(i): 0 \leqslant i<k\right\}$.

Using (84), we have

$$
\begin{aligned}
\mathbb{P}\left(\mathcal{A}_{v}(k)\right) \geqslant 1-\frac{2}{\sqrt{2 \pi}} \int_{\varepsilon \theta \chi_{v}(k)}^{\infty} \exp \left(-\frac{x^{2}}{128 r^{2 s\left(f_{v}(k)\right)}}\right) d x & \geqslant 1-2 \Delta\left(f_{v}(k)\right)^{-\frac{1}{128} \varepsilon^{2} \theta^{2}} \\
& \geqslant 1-2 \exp \left(-\frac{1}{128} 2^{-20} C^{2} 4^{k}\right) .
\end{aligned}
$$

where we have used (171), the definition of $\varepsilon(174)$, and $\chi_{v}(k)=r^{s\left(f_{v}(k)\right)} \sqrt{\log \Delta\left(f_{v}(k)\right)}$.

Clearly by choosing $C$ a large enough constant, we have

$$
\prod_{k=0}^{h_{v}-1} \mathbb{P}\left(\mathcal{A}_{v}(k)\right) \geqslant \frac{1}{2}
$$

completing the proof.

The second moment. Finally, we bound the probability of $\mathcal{E}_{u} \cap \mathcal{E}_{v}$ for $u \neq v$.

Lemma 4.10. For every $u, v \in \mathcal{L}$,

$$
\mathbb{P}\left(\mathcal{E}_{u} \cap \mathcal{E}_{v}\right) \leqslant m_{u v}^{1 / 8}\left(m_{u} m_{v}\right)^{-7 / 8} .
$$

Proof. Assume, without loss of generality, that $u \prec v \in \mathcal{L}$. It is clear from (101) that $\mathbb{P}\left(\mathcal{E}_{u}\right) \leqslant$ $m_{u}^{-7 / 8}$. Also, we have

$$
\mathbb{P}\left(\mathcal{E}_{v} \mid \mathcal{E}_{u}\right) \leqslant \mathbb{P}\left(\mathcal{A}_{v}\left(0, h_{v}-1\right), \mathcal{B}_{v}\left(0, h_{u}-1\right) \mid \mathcal{E}_{u}\right) \leqslant \prod_{k=h_{u v}}^{h_{v}-1} \mathbb{P}\left(\mathcal{B}_{v}(k) \mid \mathcal{E}_{u}, \mathcal{A}_{v}(0, k), \mathcal{B}_{v}(0, k-1)\right)
$$


Now recall that $\mathcal{E}_{u} \in \sigma\left(\mathcal{F}_{f_{v}\left(h_{u v}+1\right)}^{-}\right) \subset \sigma\left(\mathcal{F}_{f_{v}(k+1)}^{-}\right)$for all $k \geqslant h_{u v}$. By (92), we obtain,

$\prod_{k=h_{u v}}^{h_{v}-1} \mathbb{P}\left(\mathcal{B}_{v}(k) \mid \mathcal{E}_{u}, \mathcal{A}_{v}(0, k), \mathcal{B}_{v}(0, k-1)\right)=\operatorname{deg}_{\downarrow}\left(f_{v}\left(h_{v}-1\right)\right)^{-3 / 4} \prod_{k=h_{u v}}^{h_{v}-1} \operatorname{deg}_{\downarrow}\left(f_{v}(k)\right)^{-1 / 8}=m_{u v}^{1 / 8} m_{v}^{-7 / 8}$.

Altogether, we conclude that

$$
\mathbb{P}\left(\mathcal{E}_{u} \cap \mathcal{E}_{v}\right)=\mathbb{P}\left(\mathcal{E}_{u}\right) \mathbb{P}\left(\mathcal{E}_{v} \mid \mathcal{E}_{u}\right) \leqslant m_{u v}^{1 / 8}\left(m_{u} m_{v}\right)^{-7 / 8},
$$

as required.

The main coupling lemma, Lemma 4.3, is an immediately corollary of Lemmas 4.8, 4.9 and 4.10 .

\subsection{Tree-like percolation}

Lemma 4.11 below yields (76). Its proof is a variant on the well-known second moment method for percolation in trees (see [38]). First, we define a measure $\nu$ on $\mathcal{L}$ via $\nu(u)=m_{u}^{-1}$. Observe that $\nu$ is a probability measure on $\mathcal{L}$, i.e.

$$
\sum_{u \in \mathcal{L}} \nu(u)=1
$$

To see this, construct a unit flow from the root to the leaves, where each non-leaf node splits its incoming flow equally among its children. Clearly the amount that reaches a leaf $u$ is precisely $\nu(u)$.

Lemma 4.11. Suppose that to each $v \in \mathcal{L}$, we associate an event $\mathcal{E}_{v}$ such that the following bounds old.

1. $\mathbb{P}\left(\mathcal{E}_{v}\right) \geqslant \frac{1}{2} m_{v}^{-7 / 8}$ for all $v \in \mathcal{L}$.

2. $\mathbb{P}\left(\mathcal{E}_{u} \cap \mathcal{E}_{v}\right) \leqslant m_{u v}^{1 / 8}\left(m_{u} m_{v}\right)^{-7 / 8}$ for all $u, v \in \mathcal{L}$.

Define $Z=\sum_{u \in \mathcal{L}} m_{u}^{-1 / 8} \mathbf{1}_{\mathcal{E}_{u}}$. Then,

$$
\mathbb{P}(Z>0) \geqslant \frac{1}{8}
$$

Proof. By assumption (1),

$$
\mathbb{E} Z \geqslant \sum_{u \in \mathcal{L}} \frac{1}{2} m_{u}^{-1 / 8} m_{u}^{-7 / 8}=\frac{1}{2} \sum_{u \in \mathcal{L}} m_{u}^{-1}=\frac{1}{2}
$$

where the last equality follows from (102).

By assumption (2), we have

$$
\mathbb{E} Z^{2}=\sum_{u, v \in \mathcal{L}}\left(m_{u} m_{v}\right)^{-1 / 8} \mathbb{P}\left(\mathcal{E}_{u} \cap \mathcal{E}_{v}\right) \leqslant \sum_{u, v \in \mathcal{L}} m_{u v}^{1 / 8}\left(m_{u} m_{v}\right)^{-1}
$$

In order to estimate the second moment, we first fix $u$ and sum over $v$. To be more precise, let

$$
\mathcal{L}_{h}(u)=\left\{v \in \mathcal{L}: h_{u v}=h\right\},
$$


where we recall that $h_{u}$ is the height of a node $u$, and $h_{u v}$ is the height of the least-common ancestor of $u$ and $v$.

We can then partition $\mathcal{L}=\bigcup_{h \geqslant 0} \mathcal{L}_{h}(u)$ and obtain for every $u \in \mathcal{L}$,

$$
\begin{aligned}
\sum_{v \in \mathcal{L}} m_{u v}^{1 / 8} m_{v}^{-1}=\sum_{h=0}^{h_{u}} \sum_{v \in \mathcal{L}_{h}(u)} m_{u v}^{1 / 8} m_{v}^{-1} & =\sum_{h=0}^{h_{u}} \prod_{i=0}^{h-1} \operatorname{deg}_{\downarrow}\left(f_{u}(i)\right)^{1 / 8} \sum_{v \in \mathcal{L}_{h}(u)} m_{v}^{-1} \\
& =\sum_{h=0}^{h_{u}} \prod_{i=0}^{h-1} \operatorname{deg}_{\downarrow}\left(f_{u}(i)\right)^{1 / 8} \nu\left(\mathcal{L}_{h}(u)\right) .
\end{aligned}
$$

Recalling the flow representation of the measure $\nu$, we see that

$$
\nu\left(\mathcal{L}_{h}(u)\right)=\frac{\operatorname{deg}_{\downarrow}\left(f_{u}(h)\right)-1}{\operatorname{deg}_{\downarrow}\left(f_{u}(h)\right)} \prod_{i=0}^{h-1} \operatorname{deg}_{\downarrow}\left(f_{u}(i)\right) .
$$

Therefore,

$$
\sum_{v \in \mathcal{L}} m_{u v}^{1 / 8} m_{v}^{-1}=\sum_{\ell=0}^{h_{u}} \frac{\operatorname{deg}_{\downarrow}\left(f_{u}(h)\right)-1}{\operatorname{deg}_{\downarrow}\left(f_{u}(h)\right)} \prod_{i=0}^{h-1} \operatorname{deg}_{\downarrow}\left(f_{u}(i)\right)^{-7 / 8} \leqslant \sum_{\ell=0}^{h_{u}} \prod_{i=0}^{h-1} \operatorname{deg}_{\downarrow}\left(f_{u}(i)\right)^{-7 / 8} \leqslant 2,
$$

where the last transition follows from (71), for $C$ chosen sufficiently large. Applying the second moment method, we deduce that

$$
\mathbb{P}(Z>0) \geqslant \frac{(\mathbb{E} Z)^{2}}{\mathbb{E} Z^{2}} \geqslant \frac{1}{8}
$$

completing the proof.

\subsection{The local times}

We now prove Lemma 4.4, in order to the complete the analysis of the left-hand side of (63).

Lemma 4.12. Consider the local times $L_{\tau(t)}^{v}$ as defined in Theorem 1.14. For $v \in \mathcal{L}$, define

$$
\tilde{\mathcal{E}}_{v}=\left\{0<L_{\tau}^{v}(t) \leqslant 50^{2} \cdot r^{2 s\left(f_{v}\left(h_{v}-1\right)\right)} m_{v}^{-3 / 2}\right\}
$$

Then, for any $t>0$

$$
\mathbb{P}\left(\bigcup_{v \in \mathcal{L}} \tilde{\mathcal{E}}_{v}\right) \leqslant \frac{1}{16}
$$

Proof. Note that the random walk is at vertex $v_{0}$ at time $\tau(t)$. Hence, given that $L_{\tau(t)}^{v}>0$, the random walk contains at least one excursion which starts at $v$ and ends at $v_{0}$. Therefore, given that $L_{\tau(t)}^{v}>0$, we see $c_{v} L_{\tau(t)}^{v}$ stochastically dominates the random variable

$$
L=\int_{0}^{T_{v_{0}}} \mathbf{1}_{\left\{X_{t}=v\right\}} d t
$$

where $X_{t}$ is a random walk on the network started at $v$ and $T_{v_{0}}$ is the hitting time to $v_{0}$. 
By definition, every time the random walk hits $v$, it takes an exponential time for the walk to leave. Also, the probability that the random walk would hit $v_{0}$ before returning to $v$ can be related to the effective resistance (see, for example, [39]). Formally, when the random walk $W_{t}$ is at vertex $v$, it will wait until the Poisson clock $\sigma$ with rate 1 rings and then move to a neighbor (possibly $v$ itself) selected proportional to the edge conductance. Define

$$
T_{v}^{+}=\min \left\{t>\sigma: X_{t}=v\right\} .
$$

Then we have the continuous-time version of (33),

$$
\mathbb{P}_{v}\left(T_{v}^{+}>T_{v_{0}}\right)=\frac{1}{c_{v} R_{\mathrm{eff}}\left(v, v_{0}\right)} .
$$

By the strong Markov property, $L$ follows the law of the sum of a geometric number of i.i.d. exponential variables. Thus $L$ follows the law of an exponential variable with $\mathbb{E} L=c_{v} R_{\text {eff }}\left(v, v_{0}\right)$.

Recalling property (72) of our separated tree $\mathcal{T}$, we see that

$$
R_{\mathrm{eff}}\left(v, v_{0}\right)=\mathbb{E}\left(\eta_{v}-\eta_{v_{0}}\right)^{2} \geqslant 2^{-10} r^{2 s\left(f_{v}\left(h_{v}-1\right)\right)-2} .
$$

Thus,

$$
\begin{aligned}
\mathbb{P}\left(0<L_{\tau(t)}^{v} \leqslant 50^{2} \cdot r^{2 s\left(f_{v}\left(h_{v}-1\right)\right)} m_{v}^{-3 / 2}\right) & \leqslant \mathbb{P}\left(L \leqslant c_{v} \cdot 50^{2} \cdot r^{2 s\left(f_{v}\left(h_{v}-1\right)\right)} m_{v}^{-3 / 2}\right) \\
& \leqslant \frac{50^{2} \cdot r^{2 s\left(f_{v}\left(h_{v}-1\right)\right)} m_{v}^{-3 / 2}}{R_{\mathrm{eff}}\left(v, v_{0}\right)} \\
& \leqslant 2^{11} \cdot 50^{2} \cdot r^{2} m_{v}^{-3 / 2} \\
& \leqslant \frac{1}{16} m_{v}^{-1},
\end{aligned}
$$

where the last transition using (71) for $C$ chosen large enough, and $m_{v} \geqslant \exp \left(C^{2} r^{2}\right)$.

Therefore, we conclude that

$$
\mathbb{P}\left(\bigcup_{v \in \mathcal{L}} \tilde{\mathcal{E}}_{v}\right) \leqslant \frac{1}{16} \sum_{v \in \mathcal{L}} m_{v}^{-1}=\frac{1}{16},
$$

where we used, from (102), the fact that $\sum_{v \in \mathcal{L}} m_{v}^{-1}=1$, completing the proof.

\subsection{Additional applications}

We now prove a generalization of Theorem 1.7. Suppose that $V=\{1,2, \ldots, n\}$, and let $G(V)$ be a network with conductances $\left\{c_{i j}\right\}$. We define real, symmetric $n \times n$ matrices $D$ and $A$ by

$$
\begin{aligned}
& D_{i j}= \begin{cases}c_{i} & i=j \\
0 & \text { otherwise. }\end{cases} \\
& A_{i j}=c_{i j} .
\end{aligned}
$$

We write

$$
L_{G}=\frac{D-A}{\operatorname{tr}(D)}
$$

and $L_{G}^{+}$for the pseudoinverse of $L_{G}$. 
Theorem 4.13. For any connected network $G(V)$,

$$
t_{\mathrm{cov}}(G) \asymp \mathbb{E}\left\|\sqrt{L_{G}^{+}} g\right\|_{\infty}^{2}
$$

where $g=\left(g_{1}, \ldots, g_{n}\right)$ is a standard $n$-dimensional Gaussian.

Proof. If $\kappa$ denotes the commute time in $G$, then the following formula is well-known (see, e.g. [32]),

$$
\kappa(i, j)=\left\langle e_{i}-e_{j}, L_{G}^{+}\left(e_{i}-e_{j}\right)\right\rangle,
$$

where $\left\{e_{1}, \ldots, e_{n}\right\}$ are the standard basis vectors in $\mathbb{R}^{n}$. Using the fact that $L_{G}^{+}$is self-adjoint and positive semi-definite, this yields

$$
\kappa(i, j)=\left\|\sqrt{L_{G}^{+}} e_{i}-\sqrt{L_{G}^{+}} e_{j}\right\|^{2} .
$$

Let $g=\left(g_{1}, \ldots, g_{n}\right) \in \mathbb{R}^{n}$ be a standard $n$-dimensional Gaussian, and consider the Gaussian processes $\left\{\eta_{i}: i=1, \ldots, n\right\}$ where $\eta_{i}=\left\langle g, \sqrt{L_{G}^{+}} e_{i}\right\rangle$. One verifies that for all $i, j \in V$,

$$
\mathbb{E}\left|\eta_{i}-\eta_{j}\right|^{2}=\left\|\sqrt{L_{G}^{+}}\left(e_{i}-e_{j}\right)\right\|^{2}=\kappa(i, j)
$$

thus by Theorem (MM),

$$
\gamma_{2}(V, \sqrt{\kappa}) \asymp \mathbb{E} \max _{i \in V} \eta_{i}=\mathbb{E} \max _{i \in V}\left\langle g, \sqrt{L_{G}^{+}} e_{i}\right\rangle=\mathbb{E} \max _{i \in V}\left\langle\sqrt{L_{G}^{+}} g, e_{i}\right\rangle \asymp \mathbb{E}\left\|\sqrt{L_{G}^{+}} g\right\|_{\infty} .
$$

By Theorem 1.9, $\left[\gamma_{2}(V, \sqrt{\kappa})\right]^{2} \asymp t_{\operatorname{cov}}(G)$. Finally, one can use Lemma 2.2 to conclude that

$$
\left(\mathbb{E}\left\|\sqrt{L_{G}^{+}} g\right\|_{\infty}\right)^{2} \asymp \mathbb{E}\left\|\sqrt{L_{G}^{+}} g\right\|_{\infty}^{2}
$$

completing the proof.

Theorem 4.14. There a randomized algorithm which, given any connected network $G(V)$, with $m=\left|\left\{(x, y): c_{x y} \neq 0\right\}\right|$, runs in time $O\left(m(\log m)^{O(1)}\right)$ and outputs a number $A(G)$ such that $t_{\text {cov }}(G) \asymp \mathbb{E}[A(G)] \asymp\left(\mathbb{E}\left[A(G)^{2}\right]\right)^{1 / 2}$.

Proof. In [46, §4], it is shown how to compute a $k \times n$ matrix $Z$, in expected time $O\left(m(\log m)^{O(1)}\right)$, with $k=O(\log n)$, and such that for every $i, j \in V$,

$$
\kappa(i, j) \leqslant\left\|Z\left(e_{i}-e_{j}\right)\right\|^{2} \leqslant 2 \kappa(i, j) .
$$

We can associate the Gaussian processes $\left\{\eta_{i}\right\}_{i \in V}$, where $\eta_{i}=\left\langle g, Z e_{i}\right\rangle$, and $g$ is a standard $k$ dimensional Gaussian. Letting $d(i, j)=\sqrt{\mathbb{E}\left|\eta_{i}-\eta_{j}\right|^{2}}$, we see from (105) that $\sqrt{\kappa} \leqslant d \leqslant \sqrt{2 \kappa}$, therefore $\gamma_{2}(V, \sqrt{\kappa}) \asymp \gamma_{2}(V, d)$. It follows $($ see (104) $)$ that

$$
\mathbb{E}\|Z g\|_{\infty}^{2} \asymp \mathbb{E}\left\|\sqrt{L_{G}^{+}} g\right\|_{\infty}^{2} \asymp t_{\text {cov }}(G),
$$

where the last equivalence is the content of Theorem 4.13,

The output of our algorithm is thus $A(G)=\|Z g\|_{\infty}^{2}$, where $g$ is a standard $k$-dimensional Gaussian vector. The fact that $\mathbb{E}[A(G)] \asymp\left(\mathbb{E}\left[A(G)^{2}\right]\right)^{1 / 2}$ follows from Lemma 2.2 . 


\section{Open problems and further discussion}

We now present two open questions that arise naturally from the present work. The first question concerns obtaining a better deterministic approximation to the cover time.

Question 5.1. Is there, for any $\varepsilon>0$, a deterministic, polynomial-time algorithm that approximates $t_{\mathrm{cov}}(G)$ up to a $(1+\varepsilon)$ factor?

Note that the preceding question has been solved by Feige and Zeitouni [23] in the case of trees.

The second question involves concentration of $\tau_{\text {cov }}$ around its expected value. Under the assumption that $\lim _{n \rightarrow \infty} \frac{t_{\mathrm{cov}}\left(G_{n}\right)}{t_{\mathrm{hit}}\left(G_{n}\right)}=\infty$, where $t_{\text {hit }}$ denotes the maximal hitting time, Aldous [5] proves that $\frac{\tau_{\mathrm{cov}}\left(G_{n}\right)}{t_{\mathrm{cov}}\left(G_{n}\right)}$ converges to 1 in probability. We ask whether it is possible to obtain sharper concentration.

Question 5.2. Is the standard deviation of $\tau_{\text {cov }}$ bounded by the maximal hitting time $t_{\text {hit }}$ ? Furthermore, does $\frac{\tau_{\mathrm{cov}}-t_{\mathrm{cov}}}{t_{\mathrm{hit}}}$ exhibit an exponential decay with constant rate?

It is interesting to consider the extent to which Theorem 2.8 is sharp. Consider a family of graphs $\left\{G_{n}\right\}$. We point out that the asymptotic formula,

$$
t_{\mathrm{cov}}\left(G_{n}\right) \sim\left|E\left(G_{n}\right)\right| \cdot\left(\mathbb{E} \sup _{v \in V} \eta_{v}\right)^{2},
$$

holds for both the family of complete graphs and the family of regular trees, where we write $a_{n} \sim b_{n}$ for $\lim a_{n} / b_{n}=1$, and $E\left(G_{n}\right)$ denotes the set of edges in $G_{n}$. Here, $\left\{\eta_{v}\right\}$ is the GFF associated to $G_{n}$ with $\eta_{v_{0}}=0$ for some fixed vertex $v_{0}$.

To see this, note that the GFF on the $n$-vertex complete graph satisfies $\operatorname{Var} \eta_{v}=\frac{2}{n}$ and $\mathbb{E}\left(\eta_{v} \eta_{u}\right)=\frac{1}{n}$ for $v_{0} \notin\{u, v\}$. Therefore, we can write $\eta_{v}=\xi+\xi_{v}$ for every $v \neq v_{0}$, where $\xi$ and all $\left\{\xi_{v}\right\}_{v \in V}$ are i.i.d. Gaussian variables with variance $\frac{1}{n}$. It is now clear that $\mathbb{E} \sup _{v} \eta_{v} \sim \sqrt{2 \log n / n}$. Combined with the facts that $t_{\operatorname{cov}}\left(G_{n}\right) \sim n \log n$ and $\left|E\left(G_{n}\right)\right|=\frac{n(n-1)}{2}$, this confirms (106) for complete graphs.

Fix $b \geqslant 2$ and consider a regular $b$-ary tree $T_{m}$ of height $m$ with $n=\frac{b^{m+1}-1}{b-1}$ vertices. It is shown in [4] that $t_{\mathrm{cov}}\left(T_{m}\right) \sim 2 m n \log n$. On the other hand, Biggins [8] proved that the corresponding GFF satisfies $\mathbb{E} \sup _{v} \eta_{v} \sim \sqrt{2 m \log n}$. Since the number of edges in $T_{m}$ is $n-1$, we infer that (106) holds for regular trees. It is clearly very interesting to understand the generality under which (106) holds.

\section{Acknowledgements}

We are grateful to Martin Barlow and Asaf Nachmias for helpful discussions in the early stages of this work. We thank Jay Rosen and an anonymous referee for a very thorough reading of the manuscript, along with numerous insightful comments. We also thank Nike Sun, Russ Lyons, Saran Ahuja, and Yoshihiro Abe for useful comments.

\section{References}

[1] D. Aldous. Probability approximations via the Poisson clumping heuristic, volume 77 of Applied Mathematical Sciences. Springer-Verlag, New York, 1989. 
[2] D. Aldous and J. Fill. Reversible Markov Chains and Random Walks on Graphs. In preparation, available at http://www.stat.berkeley.edu/ aldous/RWG/book.html.

[3] D. J. Aldous. Markov chains with almost exponential hitting times. Stochastic Process. Appl., 13(3):305-310, 1982.

[4] D. J. Aldous. Random walk covering of some special trees. J. Math. Anal. Appl., 157(1):271283, 1991.

[5] D. J. Aldous. Threshold limits for cover times. J. Theoret. Probab., 4(1):197-211, 1991.

[6] R. Aleliunas, R. M. Karp, R. J. Lipton, L. Lovász, and C. Rackoff. Random walks, universal traversal sequences, and the complexity of maze problems. In 20th Annual Symposium on Foundations of Computer Science (San Juan, Puerto Rico, 1979), pages 218-223. IEEE, New York, 1979.

[7] M. T. Barlow, J. Ding, A. Nachmias, and Y. Peres. The evolution of the cover time. Preprint, available at http://arxiv.org/abs/1001.0609.

[8] J. D. Biggins. Chernoff's theorem in the branching random walk. J. Appl. Probability, 14(3):630-636, 1977.

[9] A. Z. Broder and A. R. Karlin. Bounds on the cover time. J. Theoret. Probab., 2(1):101-120, 1989.

[10] G. A. Campbell. Cisoidal oscillations. Trans. Amer. Inst. Elec. Engrs., (30), 1911.

[11] A. K. Chandra, P. Raghavan, W. L. Ruzzo, R. Smolensky, and P. Tiwari. The electrical resistance of a graph captures its commute and cover times. Comput. Complexity, 6(4):312$340,1996 / 97$.

[12] C. Cooper and A. Frieze. The cover time of the giant component of a random graph. Random Structures Algorithms, 32(4):401-439, 2008.

[13] D. Coppersmith and S. Winograd. Matrix multiplication via arithmetic progressions. J. Symbolic Comput., 9(3):251-280, 1990.

[14] A. Dembo, Y. Peres, J. Rosen, and O. Zeitouni. Cover times for Brownian motion and random walks in two dimensions. Ann. of Math. (2), 160(2):433-464, 2004.

[15] P. G. Doyle and J. L. Snell. Random walks and electric networks, volume 22 of Carus Mathematical Monographs. Mathematical Association of America, Washington, DC, 1984.

[16] R. M. Dudley. The sizes of compact subsets of Hilbert space and continuity of Gaussian processes. J. Functional Analysis, 1:290-330, 1967.

[17] E. B. Dynkin. Gaussian and non-Gaussian random fields associated with Markov processes. J. Funct. Anal., 55(3):344-376, 1984.

[18] E. B. Dynkin. Local times and quantum fields. In Seminar on stochastic processes, 1983 (Gainesville, Fla., 1983), volume 7 of Progr. Probab. Statist., pages 69-83. Birkhäuser Boston, Boston, MA, 1984. 
[19] N. Eisenbaum. Une version sans conditionnement du théorème d'isomorphisms de Dynkin. In Séminaire de Probabilités, XXIX, volume 1613 of Lecture Notes in Math., pages 266-289. Springer, Berlin, 1995.

[20] N. Eisenbaum, H. Kaspi, M. B. Marcus, J. Rosen, and Z. Shi. A Ray-Knight theorem for symmetric Markov processes. Ann. Probab., 28(4):1781-1796, 2000.

[21] U. Feige. A tight lower bound on the cover time for random walks on graphs. Random Structures Algorithms, 6(4):433-438, 1995.

$[22]$ U. Feige. A tight upper bound on the cover time for random walks on graphs. Random Structures Algorithms, 6(1):51-54, 1995.

[23] U. Feige and O. Zeitouni. Deterministic approximation for the cover time of trees. Preprint, available at http://arxiv1.library.cornell.edu/abs/0909.2005,.

[24] X. Fernique. Régularité de processus gaussiens. Invent. Math., 12:304-320, 1971.

[25] X. Fernique. Regularité des trajectoires des fonctions aléatoires gaussiennes. In École d'Été de Probabilités de Saint-Flour, IV-1974, pages 1-96. Lecture Notes in Math., Vol. 480. Springer, Berlin, 1975.

[26] R. M. Foster. The average impedance of an electrical network. In Reissner Anniversary Volume, Contributions to Applied Mechanics, pages 333-340. J. W. Edwards, Ann Arbor, Michigan, 1948.

[27] O. Guédon and A. Zvavitch. Supremum of a process in terms of trees. In Geometric aspects of functional analysis, volume 1807 of Lecture Notes in Math., pages 136-147. Springer, Berlin, 2003.

[28] S. Janson. Gaussian Hilbert spaces, volume 129 of Cambridge Tracts in Mathematics. Cambridge University Press, Cambridge, 1997.

[29] J. Jonasson and O. Schramm. On the cover time of planar graphs. Electron. Comm. Probab., 5:85-90 (electronic), 2000.

[30] J. Kahn, J. H. Kim, L. Lovász, and V. H. Vu. The cover time, the blanket time, and the Matthews bound. In 41st Annual Symposium on Foundations of Computer Science (Redondo Beach, CA, 2000), pages 467-475. IEEE Comput. Soc. Press, Los Alamitos, CA, 2000.

[31] J. D. Kahn, N. Linial, N. Nisan, and M. E. Saks. On the cover time of random walks on graphs. J. Theoret. Probab., 2(1):121-128, 1989.

[32] D. J. Klein and M. Randić. Resistance distance. J. Math. Chem., 12(1-4):81-95, 1993. Applied graph theory and discrete mathematics in chemistry (Saskatoon, SK, 1991).

[33] F. B. Knight. Random walks and a sojourn density process of Brownian motion. Trans. Amer. Math. Soc., 109:56-86, 1963.

[34] M. Ledoux. The concentration of measure phenomenon, volume 89 of Mathematical Surveys and Monographs. American Mathematical Society, Providence, RI, 2001. 
[35] M. Ledoux and M. Talagrand. Probability in Banach spaces, volume 23 of Ergebnisse der Mathematik und ihrer Grenzgebiete (3) [Results in Mathematics and Related Areas (3)]. SpringerVerlag, Berlin, 1991. Isoperimetry and processes.

[36] D. A. Levin, Y. Peres, and E. L. Wilmer. Markov chains and mixing times. American Mathematical Society, Providence, RI, 2009. With a chapter by James G. Propp and David B. Wilson.

[37] L. Lovász. Random walks on graphs: a survey. In Combinatorics, Paul Erdős is eighty, Vol. 2 (Keszthely, 1993), volume 2 of Bolyai Soc. Math. Stud., pages 353-397. János Bolyai Math. Soc., Budapest, 1996.

[38] R. Lyons. Random walks, capacity and percolation on trees. Ann. Probab., 20(4):2043-2088, 1992.

[39] R. Lyons, with Y. Peres. Probability on Trees and Networks. In preparation. Current version available at http://mypage.iu.edu/ rdlyons/prbtree/book.pdf, 2009.

[40] M. B. Marcus and J. Rosen. Sample path properties of the local times of strongly symmetric Markov processes via Gaussian processes. Ann. Probab., 20(4):1603-1684, 1992.

[41] M. B. Marcus and J. Rosen. Gaussian processes and local times of symmetric Lévy processes. In Lévy processes, pages 67-88. Birkhäuser Boston, Boston, MA, 2001.

[42] M. B. Marcus and J. Rosen. Markov processes, Gaussian processes, and local times, volume 100 of Cambridge Studies in Advanced Mathematics. Cambridge University Press, Cambridge, 2006.

[43] P. Matthews. Covering problems for Markov chains. Ann. Probab., 16(3):1215-1228, 1988.

[44] D. Ray. Sojourn times of diffusion processes. Illinois J. Math., 7:615-630, 1963.

[45] D. Spielman. Algorithms, graph theory, and linear equations in Laplacian matrices. To appear, Proceedings of the International Congrees of Mathematicians, Hyderabad, India, 2010.

[46] D. Spielman and N. Srivastava. Graph sparsification by effective resistances. Available at http://arxiv.org/abs/0803.0929, 2008.

[47] D. Spielman and S.-H. Teng. Nearly-linear time algorithms for preconditioning and solving symmetric, diagonally dominant linear systems. Available at http://arxiv.org/abs/cs.NA/0607105, 2006.

[48] M. Talagrand. Regularity of Gaussian processes. Acta Math., 159(1-2):99-149, 1987.

[49] M. Talagrand. Embedding subspaces of $L_{p}$ in $l_{p}^{N}$. In Geometric aspects of functional analysis (Israel, 1992-1994), volume 77 of Oper. Theory Adv. Appl., pages 311-325. Birkhäuser, Basel, 1995.

[50] M. Talagrand. Majorizing measures: the generic chaining. Ann. Probab., 24(3):1049-1103, 1996. 
[51] M. Talagrand. Majorizing measures without measures. Ann. Probab., 29(1):411-417, 2001.

[52] M. Talagrand. The generic chaining. Springer Monographs in Mathematics. Springer-Verlag, Berlin, 2005. Upper and lower bounds of stochastic processes.

[53] P. Tetali. Random walks and the effective resistance of networks. J. Theoret. Probab., 4(1):101109, 1991.

[54] P. Winkler and D. Zuckerman. Multiple cover time. Random Structures Algorithms, 9(4):403411, 1996.

[55] D. Zuckerman. A technique for lower bounding the cover time. SIAM J. Discrete Math., 5(1):81-87, 1992. 